\title{
AMERICAN PIKA (OCHOTONA PRINCEPS): PERSISTENCE AND ACTIVITY PATTERNS IN A CHANGING CLIMATE
}

\author{
A Thesis \\ Presented to \\ The Faculty of California Polytechnic State University \\ San Luis Obispo
}

\author{
In Partial Fulfillment \\ of the Requirements for the Degree \\ Master of Science in Biological Sciences
}

by

Cody Price Massing

May 3, 2012 
(C) 2012

Cody Price Massing

ALL RIGHTS RESERVED 


\section{COMMITTEE MEMBERSHIP}

TITLE:

AUTHOR:

DATE SUBMITTED:

COMMITTEE CHAIR:

COMMITTEE MEMBER:

COMMITTEE MEMBER:
American Pika (Ochotona princeps): Persistence and Activity Patterns in a Changing Climate

Cody Price Massing

May 3, 2012

John Perrine, Assistant Professor

Gita Kolluru, Assistant Professor

Emily Taylor, Associate Professor 


\begin{abstract}
American Pika (Ochotona princeps): Persistence and Activity Patterns in a Changing Climate
\end{abstract}

\author{
Cody Price Massing
}

An increasing amount of evidence suggests that as temperatures increase, montane animals are moving upward in elevation (IPCC 2007, Parmesan and Yohe 2003). As suitable habitats rise in elevation and then disappear altogether, these animals could be pushed to extinction. The American pika, Ochotona princeps, is a montane mammal that lives in western North America, usually at elevations above $1500 \mathrm{~m}$ (Smith and Weston 1990). Recent evidence suggests that pika population numbers are dropping in response to rising temperatures (Beever et al. 2010). The pika is a small herbivorous lagomorph, a relative of hares and rabbits. Its habitat is tightly restricted to talus slopes (rockfields) and the surrounding vegetation (Grayson 2005). Pikas have a high tolerance for cold temperatures, and do not hibernate during the long montane winter. However, they have very little tolerance for even mildly warm temperatures, and have been found to die when confined above ground at $25.5^{\circ} \mathrm{C}$ (Smith 1974b).

To better understand pika persistence, we resurveyed 17 historic pika sites in the Lassen Peak region of northern California in August and September, 2009. Six of the historic sites were abandoned, as well as an additional 11 of 17 new sites surveyed. At each site we collected habitat information, and analyzed the data for factors that were correlated with site occupancy. We also installed 38 iButton thermal dataloggers in abandoned and occupied pika use sites, to determine if temperature affects occupancy. The dataloggers remained in pika sites for 14 months and recorded temperature every 1.5 hours. Abandoned pika sites had higher average temperatures and more days below $0^{\circ} \mathrm{C}$. They also had greater shrub cover, less forb and graminoid cover, and a greater percentage of litter substrate. These findings suggest that the current warming trend may be having a negative impact on pikas in the Lassen Peak Region. As temperatures rise, pikas may be declining due to unsuitable temperatures and altered vegetative communities.

In addition to the Lassen surveys, I investigated pika behavior in different temperature regimes in the Sierra Nevada. If pikas are able to adapt to climate change, it is possible that populations of pikas in different temperature regimes may exhibit behavioral plasticity, or have evolved genetic differences, such that these populations have different daily activity schedules. To determine if there is a difference in pika behavior at different elevations I observed pikas in one low and one high elevation site within the Bishop Creek drainage system in the Sierra Nevada. I conducted behavioral observations of pikas in four time blocks throughout the day in August and September, 2010. I recorded specific behaviors, such as foraging and haying (vegetation collecting), and compared these activities between low and high elevation pikas at different times of day. In August, pikas in the low elevation site exhibited a different activity profile than 
those in the high elevation site. Low elevation pikas were significantly more crepuscular (most active at dawn and dusk) during this month. I also observed more foraging behavior in the high elevation than the low elevation site, in both August and September. Reduced activity at higher temperatures may have negative impacts on pikas as temperatures increase. Low elevation pikas may be stressed due to reduced time spent foraging and haypile (overwinter vegetation cache) gathering. However, if pikas were able to switch their activity schedules to a more nocturnal schedule, they could escape higher daytime temperatures. To detect the possibility of nocturnal behavior in low elevation pikas, I set up four infra-red remote cameras in the low elevation site. I had variable success in capturing pika behavior with the cameras, and detected no evidence of nocturnal behavior. More research on the possibility of nocturnal behavior in pikas would be worthwhile, in part to determine what chance, if any, pikas have of adapting to rising global temperatures. 


\section{ACKNOWLEDGEMENTS}

I would like to thank my adviser, John Perrine, as well as my committee members, Gita Kolluru, and Emily Taylor for their help with project design, data analysis, and thesis edits. Additionally, many thanks go to Rob Klinger, an ecologist for the U.S. Geological Survey for his help with field resources, project design, and data analysis. I especially would like to thank all of the field assistants on this project, Laurel Triatik, Stacy Huskins, Mark Wilder, and Bridgett Benedict. Additionally, I would like to thank several friends, teachers, and coworkers for their advice and support throughout the entire project, in particular, Andrew Schaffner, Jen Chase, Michaela Koenig, Elaine Chow, Tina Wang, and Francis Villablanca. For monetary support on the project, thanks go to California Polytechnic University Student Fees, National Climate Change and Wildlife Science Center (NCCWSC), and the USGS, Biological Resources Division, Western Ecological Research Center. 


\section{TABLE OF CONTENTS}

Page

CHAPTER 1. Introduction: Ochotona princeps Literature Review.............................. 1

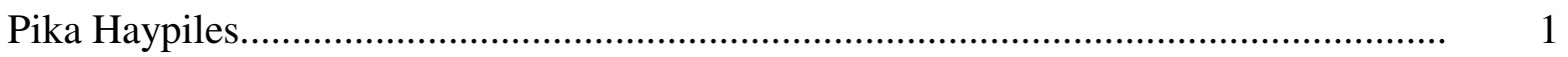

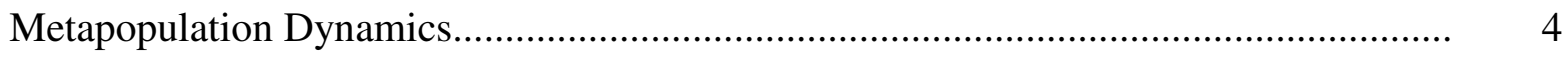

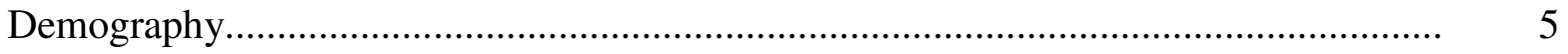

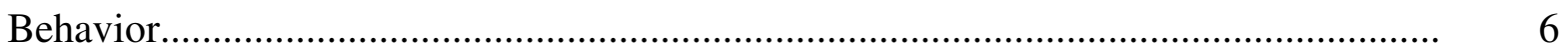

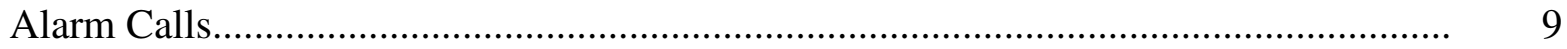

Current Research...................................................................................... 11

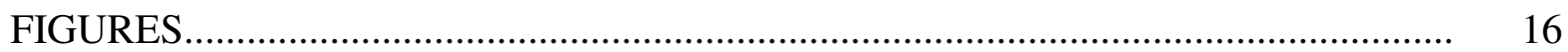

Figure 1. Species range of the American Pika, Ochotona princeps.................... 16

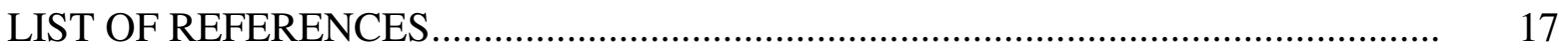

CHAPTER 2. Habitat and Temperature Profiles of Historic and Current Pika

Sites in the Lassen Peak Region.................................................................... 23

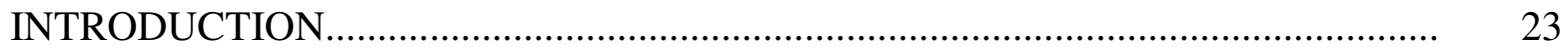

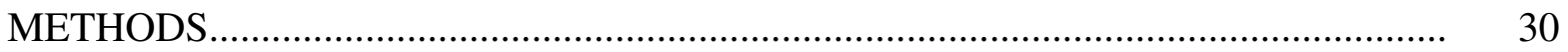

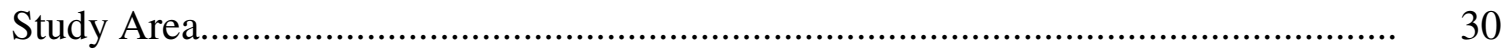

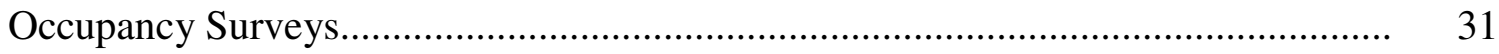

Temperature Data Collection..................................................................... 32

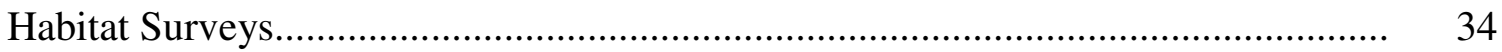

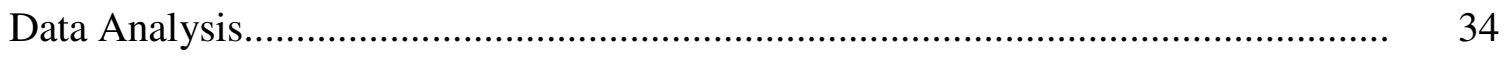

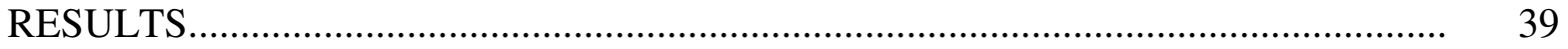




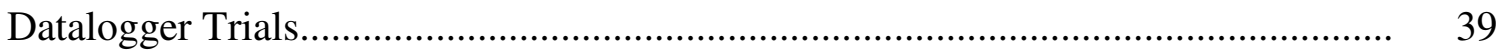

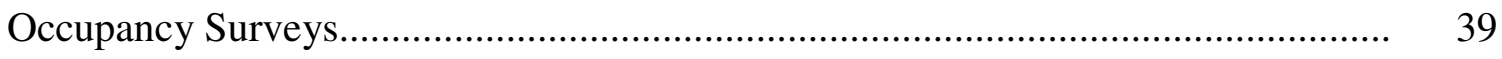

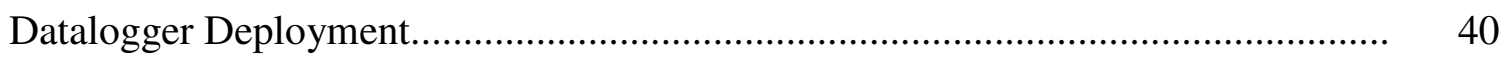

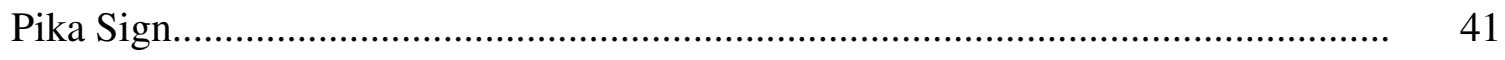

Habitat Characteristics......................................................................... 42

Microclimate Characteristics.................................................................. 42

Logistic Regression Models........................................................................ 44

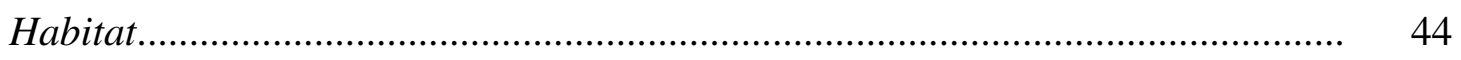

Microclimate ..................................................................................... 44

Final Models...................................................................................... 45

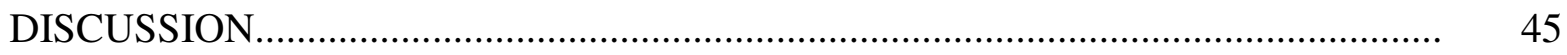

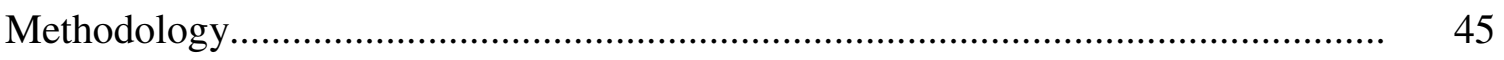

Pika Site Occupancy.............................................................................. 50

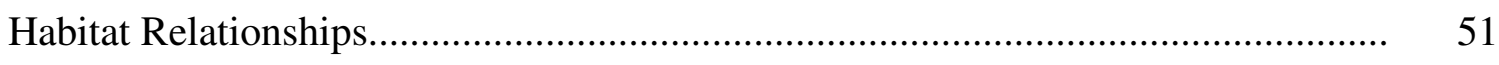

Microclimate Relationships................................................................... 57

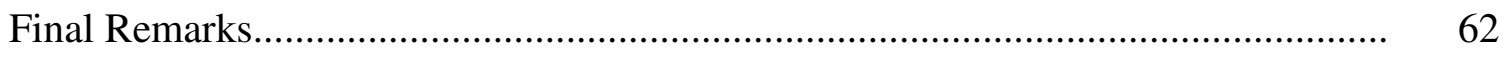

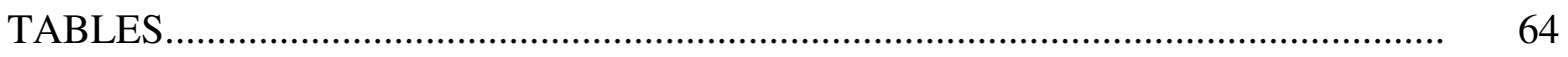

Table 1. Habitat characteristics investigated, with categorization shown............... 64

Table 2. Microclimate characteristics investigated, with categorization shown........ 64

Table 3. Correlation matrix of habitat variables, using Pearson product-moment

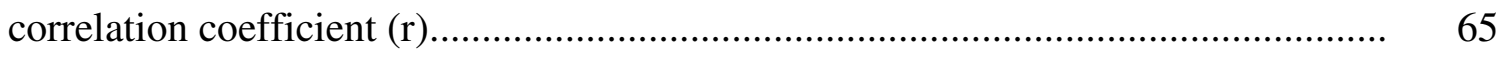

Table 4. Correlation matrix of microclimate variables, using Pearson product-

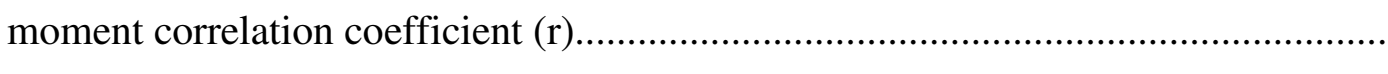


Table 5. Sites surveyed in 2009 and 2010.

Table 6. Number of historic and additional (opportunistic) sites surveyed for pikas, including those with dataloggers and those without, showing pika occupation status.

Table 7. Detection of pika sign in surveys

Table 8. Average habitat variables in occupied and abandoned sites

Table 9. Averages of microclimate variables in occupied and abandoned sites (temperatures are in Celsius) with standard error (SE)

Table 10. Results of univariate logistic regressions for habitat variables, predicting pika occupancy

Table 11. Candidate habitat models used to predict pika occupancy in additive logistic regressions

Table 12. Results of univariate logistic regressions for microclimate variables, predicting pika occupancy.

Table 13. Candidate microclimate models used to predict pika occupancy in logistic regression

Table 14. Final models used to predict pika occupancy in logistic regression, combining microclimate and habitat characteristics

Figure 1. Lassen Transect.

Figure 2. Map of Great Basin

Figure 3. The four regions investigated in the Lassen Peak study area

Figure 4. Perched pellets. 
Figure 5. Settled pellets.

Figure 6. Copper capsule used to protect iButton thermal datalogger.

Figure 7. Datalogger trials between a datalogger in a copper capsule and a datalogger with no capsule

Figure 8. Datalogger trials between a datalogger in a copper capsule and a

datalogger with no capsule

Figure 9. Survey sites in the Lassen Peak region, showing both occupied (blue)

and abandoned (red) pika sites.

Figure 10. Average $( \pm \mathrm{SE})$ relative cover of vegetation classes in abandoned and occupied pika sites

Figure 11. Yearly temperature profile of Bluff Falls, near Mineral, an abandoned site (elevation $2021 \mathrm{~m})$

Figure 12. Yearly temperature profile of Paradise Meadow, LVNP, an occupied site (elevation $2253 \mathrm{~m}$ )

Figure 13. Yearly temperature profile of Black Mountain, near Eagle Lake, an abandoned site (elevation $1865 \mathrm{~m}$ ).

Figure 14. Average temperatures $( \pm \mathrm{SE})$ in abandoned and occupied sites, measured by thermal dataloggers approximately $1 \mathrm{~m}$ in talus matrix in 2009 and 2010

Figure 15. Mosquito pond datalogger site.

LIST OF REFERENCES

CHAPTER 3. Activity Profiles of High and Low Elevation Pikas in the

Sierra Nevada. 


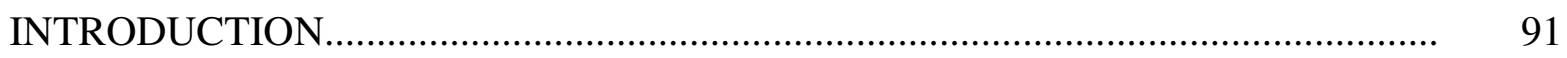

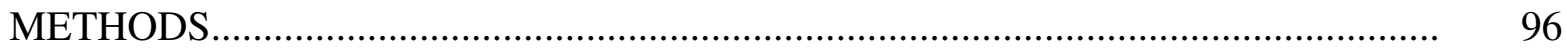

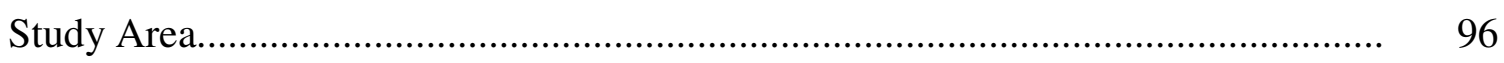

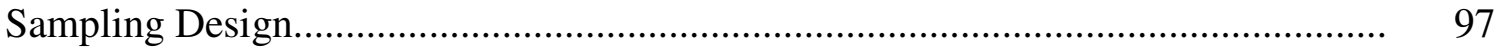

Activity Profiles............................................................................................. 98

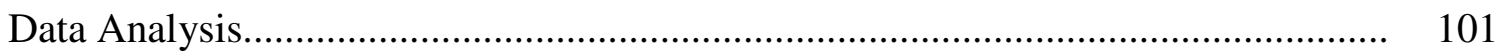

Temperature

Activity Rates......................................................................................... 101

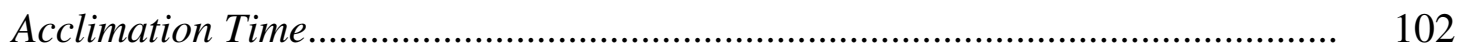

Activity Analysis........................................................................................ 103

Remote Camera Placement.......................................................................... 104

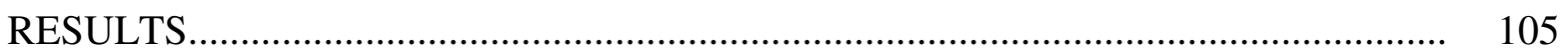

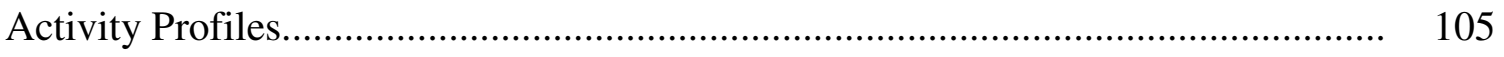

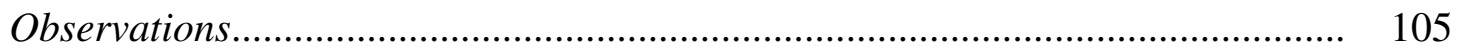

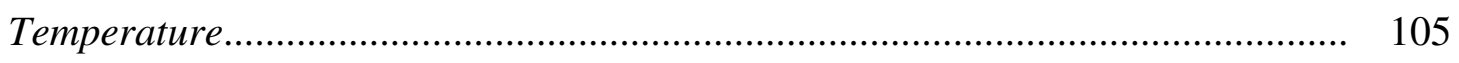

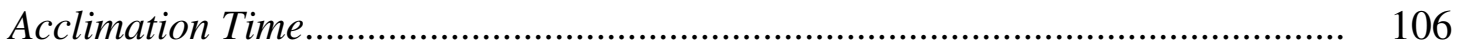

Activity Rates.......................................................................................... 106

Rates of Specific Activities....................................................................... 107

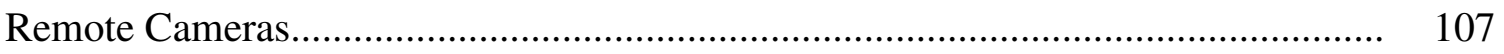

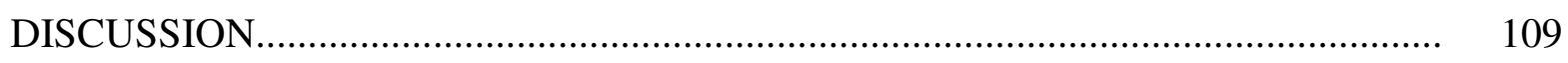

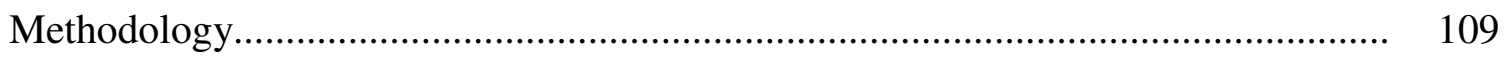

Activity Profiles and Relationship to Temperature................................................. 110

Specific Activities and Seasonality.................................................................... 114 
Nocturnal Behavior.

Final Remarks.

TABLES.

Table 1. Pika behaviors recorded.

Table 2. Minutes of observation and total numbers of pikas observed in both the high (3321 m) and low (2616 m) elevation sites during August and September.......

Table 3. Average temperatures $( \pm \mathrm{SE})$ of high $(3321 \mathrm{~m})$ and low $(2616 \mathrm{~m})$

elevation sites in August and September. Sample sizes (n) are the total number of sampling observations used in the analysis

Table 4. Average activity rates $( \pm \mathrm{SE})$ of pikas in high $(3321 \mathrm{~m})$ and low $(2616 \mathrm{~m})$ elevation sites during August and September sampling periods

Table 5. Results of GLMMs predicting pika aboveground activity rate, accounting for plot and subplot as nested random effects

Table 6. Results of GLMMs predicting pika forage rate, accounting for plot and subplot as nested random effects

Table 7. Results of GLMMs predicting pika hay rate, accounting for plot and subplot as nested random effects

Table 8. Results of GLMMs predicting pika run rate, accounting for plot and subplot as nested random effects

Table 9. Results of GLMMs predicting pika perch rate, accounting for plot and subplot as nested random effects

Table 10. Results of GLMMs predicting pika groom rate, accounting for plot and subplot as nested random effects 
Figure 1. Map of study area, showing plots in both high (3321 m) and low (2616

m) elevation sites.

Figure 2. Map of plots 6 and 7, in the high elevation site, near Loch Leven in the Inyo National Forest

Figure 3. Average temperatures $( \pm \mathrm{SE})$ on shaded talus surfaces during pika observation sessions in August and September, in the high (3321 m) and low (2616 m) elevation site throughout the day, from time blocks 1-4 (dawn to dusk).

Figure 4. Average pika activity rates $( \pm$ SE) during the first five, second five (10, graph), and final ten minutes (20, graph) of observation sessions.

Figure 5. Average pika activity rates $( \pm \mathrm{SE})$ in samples without an acclimation period, $\mathrm{N}$ (observers waited at least ten minutes before observations began), and samples with an acclimation period, Y (observers began sampling immediately upon arrival to slope)

Figure 6. Average pika aboveground activity rate $( \pm \mathrm{SE})$ throughout the day in August and September in a high (3321 m) and a low (2616 m) elevation site.

Figure 7. Average aboveground activity rates $( \pm \mathrm{SE})$, per $30 \mathrm{sec}$, of pikas in the high (3321 m) and low (2616 m) elevation sites during August and September

Figure 8. Rates of specific activities $( \pm \mathrm{SE})$ in the high $(3321 \mathrm{~m})$ and low $(2616 \mathrm{~m})$ elevation sites, in August and September. 
Figure 9. Observed rates of foraging $( \pm \mathrm{SE})$ in high $(3321 \mathrm{~m})$ and low $(2616 \mathrm{~m})$ elevation sites in time blocks 1-4 (dawn to dusk).

Figure 10. Observed rates of haying $( \pm \mathrm{SE})$ in high $(3321 \mathrm{~m})$ and low $(2616 \mathrm{~m})$

elevation sites during August and September.....

Figure 11. Observed rates of running $( \pm \mathrm{SE})$ in high $(3321 \mathrm{~m})$ and low $(2616 \mathrm{~m})$ elevation sites in time blocks 1-4 (dawn to dusk)

Figure 12. Observed rates of perching $( \pm \mathrm{SE})$ in high $(3321 \mathrm{~m})$ and low $(2616 \mathrm{~m})$ elevation sites in time blocks 1-4 (dawn to dusk).

Figure 13. (A) Camera placed approximately $4 \mathrm{~m}$ from area of high activity, resulted in successful pika pictures. (B) Successful pika picture resulting from this camera.

Figure 14. Camera placed approximately $1 \mathrm{~m}$ from haypile

Figure 15. Successful pika picture. 


\section{CHAPTER 1.}

\section{Introduction: Ochotona princeps Literature Review}

The American pika, Ochotona princeps, is a small (121-176 g), herbivorous lagomorph that lives in mountainous areas in western North America (Smith and Weston 1990). There are five subspecies of $O$. princeps, separated geographically (Hafner and Smith 2010). American pikas are found from south-central California, east to Colorado, and north to central British Columbia (Figure 1). They typically live in alpine or subalpine environments, and are indicators of cool, mesic microclimates (Hafner 1993). In the western Cascade Range, pikas have been found as low as $40 \mathrm{~m}$ (Roest 1953, as cited by Verts and Carraway 1998), but in the southern part of their range pikas are believed to live mainly at elevations above $2500 \mathrm{~m}$ (Smith and Weston 1990). In all areas of their range, pikas inhabit areas of broken rock, or talus (Smith and Weston 1990).

\section{Pika Haypiles}

Pikas are generalist herbivores, and eat a variety of sedges, grasses, forbs, shrubs, and even conifers and lichens (Conner 1983, Dearing 1997, Millar and Zwickel 1972). They do not hibernate during the long montane winter; instead they survive on stores of cached vegetation, called haypiles, which they stockpile during the summer and autumn (Smith and Weston 1990). During the haying season, from late July through September, pikas spend approximately $25-55 \%$ of surface activity time collecting haypiles, and they may make up to 27 haying trips per hour (Conner 1983, Smith and Ivins 1983). Haying 
is risky and energetically demanding behavior. Haying trips tend to be significantly longer distances than foraging trips (Huntly et al. 1986), and they expose pikas to greater predation risk (Morrison et al. 2004). Haypile size varies by location and individual (Millar and Zwickel 1972). The average sizes of haypiles in two sites in the Rocky Mountains in Colorado ranged from 20 to $28.3 \mathrm{~kg}$ of fresh vegetation per pika (Dearing 1997).

Despite the large amount of effort pikas expend to collect haypiles, there is some evidence that haypiles are not always necessary for survival (Conner 1983, Millar and Zwickel 1972). The full usefulness of the haypile is questionable, and is likely location specific (Dearing 1997). Many pikas do not collect haypiles at all (Beever et al. 2008, Ray and Beever 2007), and those that do collect them do not always use them (Conner 1983), or collect similar amounts of vegetation (Millar and Zwickel 1972). The haypile likely functions as a food source backup, in case winter conditions are harsh enough to prevent winter foraging (Conner 1983, Wilkening et al. 2011), but in some regions it may be necessary for overwinter survival every year (Dearing 1997).

A common misconception in haypile collection stems from a misinterpretation of the study performed by Millar and Zwickel (1972), reported by Smith and Weston (1990) and Conner (1983). The misinterpretation is attributed to "Millar and Zwickel 1972" by researchers who apparently read the more recent sources, but did not refer back to the original paper. Millar and Zwickel (1972) measured the haypile sizes of pikas in different habitat types, and found that all populations had similar overwinter survival rates, despite the fact that haypiles were drastically different in size depending on the habitat type. Smith and Weston (1990) describe this study erroneously, "Millar and 
Zwickel (1972b) experimentally removed entire haypiles in autumn, and survivorship to the following spring did not differ from the control group in which haypiles were left intact." As published in the cited paper, no experiment of this type was performed, yet some current publications state that it was (e.g., Wilkening et al. 2011). Dearing (1997), in her paper on pika haypiles, pointed out this mistake, and although her paper is widely cited, the misinterpretation is still reported.

Another misconception about pika haypiles that is reported in some field guides (e.g., Jameson and Peeters 2004, Laws 2007), but has repeatedly been unsupported in primary literature is the idea that pikas cure, or dry, hay on the surface before stowing it underground at the onset of winter. Researchers widely agree that pikas stash vegetation in rock crevices, sometimes partially on the surface, but do not move the vegetation after caching. Haypiles seen the previous fall and summer remain in the same location at the time of snowmelt (Millar and Zwickel 1972, Dearing 1997, Krear 1965). However, pikas may have other ways of preserving vegetation during the winter. Dearing (1997) found that pikas preferentially stored a plant with antibacterial properties, Acomastylis rossii. In addition, the plants that pikas selected for storage decayed much slower while retaining the same amount of nutrition as plants selected for immediate consumption in summer (Dearing 1997). Most of the plants stored in haypiles contain high concentrations of phenolics, which makes them both slower to decompose and more difficult to digest. Approximately halfway through winter, toxic secondary compounds in stashed vegetation degrade enough for pikas to ingest the material (Dearing 1997). This may explain why pikas have been seen foraging on lichens and tree bark during the first half of winter, and 
then disappear underground at approximately the same time that haypiles are least toxic (Dearing 1997, Conner 1983).

\section{Metapopulation Dynamics}

American pikas live in talus slopes or broken rock next to suitable vegetation. Occasionally they inhabit manmade structures, such as mine tailings and lumber piles (Smith and Weston 1990). They are usually found in broken rock that is 0.2 to $1 \mathrm{~m}$ in diameter (Tyser 1980). Because areas of talus tend to be patchily distributed over a landscape, these patches of talus effectively act as islands, separating pika demes from each other. In fact, pikas are considered to have a classic metapopulation structure (Moilanen et al. 1998). They are highly reliant on patches of talus, which are separated by meadows or other areas of vegetation, and they rarely move more than six meters from the edge of the talus to forage (Franken and Hik 2004, Huntly et al. 1986, Roach et al. 2001). There is only limited dispersal between patches. Juveniles can apparently disperse only very short distances, likely because they are more susceptible to predation the longer they are away from talus. The farther apart these patches of talus, the less chance there is of dispersal between patches (Franken and Hik 2004, Smith 1974a).

The model that best depicts a metapopulation of pikas is dependent on the landscape, so it varies in different areas. In some populations, a stepping stone metapopulation model best portrays the population structure, while in others it appears that the mainland-island, or source-sink, model is a better fit (Peacock and Smith 1997). Kreuzer and Huntly (2003) studied a population of pikas in Wyoming, which contained snowbound demes and meadow-bound demes. They found that pikas appeared to be 
functioning under source-sink dynamics (Kreuzer and Huntly 2003). The meadow demes had higher reproductive rates and more emigration, and thus were believed to be sources, while the snow demes had low reproductive rates and high immigration rates, and were believed to be sinks (Kreuzer and Huntly 2003).

\section{Demography}

Mortality rates are highest among pikas at two life stages: from birth to 1 year, and from 5-7 years of age, 7 being their maximum age limit. Populations grow in the summer when pups are born and decrease in winter, likely due to high mortality rates at that time (Smith 1978). It must be noted, however, that during winter pikas are significantly understudied. It has been inferred that there are high overwinter mortality rates because populations tend to be smaller in spring than the previous fall at the time of the last count, but the cause of this reduction is difficult to determine. However, more evidence suggests that acute cold stress caused by reduced snowpack may be a major cause of pika mortality (Beever at al. 2010, Ray 2006, Smith and Ivins 1983).

Pikas are philopatric, and juveniles disperse to the nearest open available territory. If the natal patch is currently full, juveniles disperse to other patches (Peacock and Smith 1997). Because of their sensitivity to warm temperatures, and the increased probability of predation the longer they are away from talus, dispersal distances are effectively limited (Smith 1974a, b). In one study in Bodie, California the average dispersal distance for marked juveniles was $91.25 \mathrm{~m}$, which included dispersal within the natal population patch (Peacock and Smith 1997). However, Peacock (1997) found little genetic 
differences between populations $2 \mathrm{~km}$ apart, indicating that pikas may disperse at least that distance.

Juvenile pikas reach adult size after three months. They breed the following year in late spring or early summer. Female pikas have two litters a year, each on average about 2-3 pups, but the second litter is frequently not successful (Smith 1978, Smith and Ivins 1983). It appears that second litters usually do not survive because they are abandoned during weaning. Smith (1978) found that only 7-10\% of second litters survived weaning. It is unknown why females have second litters given the high probability of abandonment (Smith 1978). The second litter may serve as a backup; when the first litter is preyed upon or disappears, second litters tend to be more successful (Smith 2008).

\section{Behavior}

American pikas are territorial and considered nonsocial. Most interactions between conspecifics are aggressive, and usually involve a territory holder chasing out an intruder (Smith 2008). The purpose of their extreme territoriality appears to be to guard haypiles. Pikas are most territorial when they are collecting haypiles (Kawamichi 1976), and they also have smaller home range diameters during this time, so they can better guard their haypiles (Smith and Ivins 1983). Some researchers have observed pikas stealing from each other's haypiles (Kawamichi 1976, McKechnie et al. 1994). This kleptoparasitism does not appear to occur frequently, however, or in every population studied. In fact, the highest rate of kleptoparasitism recorded was just 3.6\% (this percentage is the number of thefts out of the total number of haying trips observed over 
the course of a one year study, McKechnie et al. 1994). Aggressive behavior seems to deter kleptoparasites, and this is evidenced by the fact that many robbing expeditions take place when the resident is underground (78\% as recorded by McKechnie et al. 1994). To test whether guarding haypiles deters robbers, McKechnie et al. (1994) experimentally placed seven haypiles in unguarded areas on the talus. These haypiles were quickly depleted by the surrounding pikas, within 25 minutes in one case, and all within 48 hours. Interestingly, if there is kleptoparasitism in a population, it seems to be exhibited by only one or two individuals in that population, and these individuals are repeat offenders (Kawamichi 1976, McKechnie et al. 1994).

When a resident pika discovers that an invader has moved into its territory, it will typically chase the invader away. However, residents frequently do not detect invaders, either because the residents are underground, or because their view is impeded by tall boulders (Kawamichi 1976, Svendsen 1979). Kawamichi (1976) determined the dominance hierarchy in a population by observing whether an occupant was successful in chasing away an invader, and the rapidity with which they did so. He found that adults were dominant to juveniles, and adult males were dominant to females. This ranking has been confirmed by a captive study (Whitworth and Southwick 1984). Several studies have shown that juveniles occupy less optimal habitat, including areas with smaller rocks, fewer rocks, or simply a smaller area (Kawamichi 1976, Lutton 1975, Smith and Ivins 1983).

Neighboring pikas are nearly always of opposite sex. This pattern appears to be maintained; when a territory is vacated and a new pika moves in, the new occupant tends to be the same sex as the previous occupant (Smith 2008). This settlement pattern may 
be maintained by the resident adults, who have been found to be more aggressive toward juveniles of the same sex than the opposite sex (Smith and Ivins 1983). It may be easier for a juvenile male, for instance, to settle into a territory next to an adult female than to settle next to an adult male, because he will receive less aggression from the female.

Although pikas are by and large solitary animals, nearest neighbors appear to be most tolerant of each other's presence. When a territory holder encounters an intruder from a non-adjacent territory, the intruder is nearly always chased. However, when a territory holder encounters a neighbor, chases occur about half of the time (Smith 2008). Smith and Ivins (1983) caught and marked pikas and observed their interactions for three years. They found that when affiliative behavior, or social tolerance, was observed it tended to be between neighbors ( $86 \%$ of the time), and that these neighbors were usually of opposite sex, implying that they were mated pairs. Further support for this idea comes from Peacock (1997) who used genetic methods to determine parentage and found that the average distance between mates was 1.38 home ranges. This indicates that pikas tend to mate with the closest available neighbor of the opposite sex.

American pika populations tend to have approximately equal sex ratios (Smith and Weston 1990), and individuals in these populations are evenly spaced, alternating male and female (Smith and Ivins 1983). Because of these factors and the low resource abundance in their alpine habitat, American pikas have a facultative monogamous mating system (Smith and Ivins 1983, Smith 2008). There is no sexual dimorphism, and while males have somewhat larger home ranges (Kawamichi 1976), they are unable to control large enough territories to guard more than one female. Social repulsion between females further ensures that females cannot be "clumped" and guarded (Smith and Ivins 1983). 
Peacock (1997) found that out of twelve mated pairs, one male and one female had multiple mates. The male mated with four different females over a two-year period, and these females were between zero and four home ranges away from his territory.

Further evidence for monogamy in pikas comes from Kawamichi (1976). He found that the most frequent overlaps in home ranges were between males and females, and these overlaps were more frequent than overlap between neighbors. Conspicuous overlap between certain males and females allowed Kawamichi to infer that these pikas were paired, and would likely be mates in the next breeding season. Although this seems somewhat unsubstantiated, it makes sense when some other species of pikas are considered. Several Asian species of pikas demonstrate clear single mate pairing, and some, such as the Japanese pika, O. hyperborea, appear to collect haypiles and to winter as a pair (Kawamichi 1976). On the other hand, plateau pikas, O. curzoniae, which live on the Tibetan Plateau, exhibit almost all types of mating systems: monogamy, polygyny, polyandry, and polygynandry. The type of mating system each group employs apparently depends on the sex ratio (Smith 2008).

\section{Alarm Calls}

American pikas give distinctive alarm calls, and the timing and frequency of their calls varies between populations (Conner 1982, Trefry and Hik 2010). They give two types of calls, a long call and a short call, the latter being the best studied. The short call functions as an attention getter; when an individual calls surrounding pikas stop their activities and look in the direction of the caller (Svendsen 1979). The call is used in a variety of situations, including territorial chases, warning of a predator (Conner 1982), in 
response to conspecific vocalization or movement, and from a rocky perch just before or after movement (Ivins and Smith 1983). Ivins and Smith (1983) found that pikas called with greater intensity and for longer periods when predators were present. The number of short calls per minute and the length of calling were greater for martens and weasels than for non-predators, such as marmots and deer, indicating that pikas were able to differentiate between predators and non-predators.

Several observers have noticed that pikas call less frequently to weasels than they do to martens (Ivins and Smith 1983, Lutton 1975). Weasels are believed to be more of a threat to pikas because they can follow them into rock crevices, while martens cannot. Therefore, a pika is more likely to be preyed upon when calling to warn of a weasel than a marten (Ivins and Smith 1983). Ivins and Smith (1983) investigated this behavior, and they found that only adults discriminated between martens and weasels in calling frequency, and of these males had a more discriminatory response, calling less often than females when weasels were present. Additionally, Ivins and Smith (1983) found that juvenile pikas did not discriminate between predators and non-predators in their call duration or frequency, and also did not exhibit the same adult avoidance behaviors. In general, juveniles called far less often than adults. Either calling is a learned behavior in pikas, or the tendency for calling in juveniles has been selected against. Because calling does increase the risk of predation to the caller (Ivins and Smith 1983), it would be in a juvenile's best interest not to call. 


\section{Current Research}

Recently, American pikas have gained attention as possible indicators of climate change, in part because they are believed to be poorly adapted to high temperatures (Smith 1974b). They rely on talus not only for protection from predators, but also for thermal cover. Pikas are well adapted to surviving cold temperatures, but they cannot regulate their body temperature to warm temperatures physiologically, so they must thermoregulate behaviorally. They tend to remain under talus and be less active on warmer days (Smith 1974b, MacArthur and Wang 1974). In lower elevation sites they may come out of their rock burrows only during the morning and evening hours (Smith 1974b). Studies show that pikas will die if confined above ground at temperatures as low as $25.5^{\circ}-28^{\circ} \mathrm{C}$ (MacArthur and Wang 1973, Smith 1974b). In comparison with other lagomorphs, pikas have a high basal metabolic rate (143\% of that predicted by allometric

models, Katzner et al. 1997), and consequently an extremely high mean body temperature (40.1 ${ }^{\circ} \mathrm{C}$, MacArthur and Wang 1973). There is only a small difference between the pika's mean body temperature and its upper lethal temperature (MacArthur and Wang 1973), which makes it highly vulnerable to heat stress. Because of the pika's susceptibility to higher temperatures and climate change predictions, many researchers are currently studying pikas to determine local and regional population statuses as well as the pika's climate relationships.

The common method for estimating pika presence at a location is to search an area of suitable habitat (e.g. any broken rock or pile of debris) for sign left by pikas (Beever et al. 2003, 2008, Millar and Westfall 2010, Rodhouse et al. 2010, Shardlow et al. 2009, Smith 1974a). Pikas are highly detectable (>90\%) using these methods (Beever 
et al. 2003, 2008; Millar and Westfall 2010; Rodhouse et al. 2010). Millar and Westfall (2010) suggest beginning a search for pika sign near the borders of talus and meadows, and focusing primarily on large boulders with smaller "sentry" rocks underneath (usually conical rocks about $20 \mathrm{~cm}$ in diameter, Millar 2011). Pika sign includes feces, small spherical pellets $(2-3 \mathrm{~mm})$ that are easily recognizable (Nichols 2010), and haypiles. Haypiles are typically stored under large boulders and are partially exposed on the talus (Dearing 1997). During surveys, pikas can also be directly observed. Pikas are widely agreed to become accustomed to human presence after only a brief acclimation time (Hayes and Huntly 2005, Holmes 1991, Huntly et al. 1986, Ivins and Smith 1983, Lutton 1975, Roach et al. 2001). Furthermore, when alerted to the presence of humans, pikas frequently give a distinctive alarm call (Smith and Weston 1990).

In addition to classifying a site as currently occupied by pikas, it is also possible to classify it as previously occupied, based on sign (Millar and Westfall 2010, Nichols 2010). Sites can then be rated as either occupied or abandoned, using sign and direct observations of pikas. Occupied sites contain direct sightings and vocalizations, as well as fresh pellets, and sometimes green vegetation in haypiles. Abandoned sites contain only white, decomposing pellets; white, chalky urine stains; and sometimes old haypiles of stick pieces (Millar and Westfall 2010).

Recent resurveys in the Great Basin indicate that pika populations may be declining in response to rising temperatures (Beever et al. 2010). In the late 1990s Beever et al. (2003) performed a resurvey of the Great Basin, based on surveys from the early 1900s. They discovered that 6 of 25 populations of pikas had gone extinct, and many of the remaining populations had moved to higher elevations. The extirpated 
populations were located at significantly lower elevations, with higher average maximum summer temperatures. In subsequent sampling, Beever et al. (2010) discovered that three more of the previously occupied populations had been extirpated between 2000 and 2007. They found that chronic heat stress and acute cold stress were the best predictors for extirpation of a site. Chronic heat stress (average summer temperature) was higher in extirpated sites, and acute cold stress (number of days below $-5^{\circ} \mathrm{C}$ ) was also higher in abandoned sites. Additionally, extirpated sites contained a lower forb cover than persistence sites (Wilkening et al. 2011).

A few researchers are currently studying pikas in California, but there is still much research to be done in that region. Moritz (2007) reported lower elevational range retractions of pika populations in Yosemite National Park based on surveys from the early 1900s. These latter results are debatable, however, because only one historic site had been extirpated, and there remains one low elevation historic site that has yet to be surveyed (J. Perrine, pers. comm.). Millar and Westfall (2010) opportunistically surveyed sites in the Sierra Nevada and Great Basin for pikas in the summers of 2007 and 2008. They found pikas in more variable habitats than had previously been recorded, and at elevations ranging from $1827 \mathrm{~m}$ to $3887 \mathrm{~m}$. The lower elevation was the lowest elevation ever documented for pikas in this area of their range, and $8 \%$ of the occupied sites were below the accepted regional lower limit of $2500 \mathrm{~m}$. Their results suggest that pikas in California persist in habitats previously unexpected and understudied, and that more research is needed in this area of their range.

Concerns about the effect of global warming on pikas prompted the Center for Biological Diversity to petition the California Fish and Game Commission and the United 
States Fish and Wildlife Service (USFWS), requesting pikas be listed as threatened or endangered (Wolf et al. 2007a, b). Both agencies denied the pikas listing, largely due to a lack of data on the current distribution and abundance of these animals throughout their range (Gustafson and Logsdon 2007, USFWS 2010).

The pika's inability to regulate body temperature during heat is perhaps the trait that makes them most vulnerable to climate change. It puts lower elevation pikas, particularly those in the southern portion of their range, at elevated risk. Pikas in these areas that are living in patches of more isolated talus, or in patches that are already at the upper elevational limit of a mountain, are at even greater risk because of their limited dispersal abilities. If the environment warms too much, it is possible that temperatures in the shade of the talus could go above the lethal limit. In addition, higher temperatures could decrease the amount of time pikas can spend foraging, preventing them from gaining sufficient body mass and from collecting enough hay for overwintering (Beever et al. 2003, Wilkening et al. 2011). Rising temperatures may also cause a decrease in snowpack levels (IPCC 2007), and this may have a detrimental effect on pikas. The loss of the insulating snowpack layer exposes pikas to lower temperatures, and may negatively impact their survival (Beever et al. 2010, Ray 2006).

Using projections from 16 global climate models and three emissions scenarios, Loarie et al. (2009) estimated a greater than 50\% probability of extirpations of pikas in 15-59\% of their range by the year 2099. In the Great Basin in particular, McDonald and Brown (1992) predicted that if the average temperature rises $3^{\circ} \mathrm{C}$, pikas will go extinct in four out of five mountain ranges they currently inhabit in that region. Additionally, McDonald and Brown (1992) predicted that the pika will fare worse than 10 out of 13 
montane mammals in the Great Basin. If the average temperature rises higher still, as it is predicted to, the survival of pikas in the Great Basin is extremely uncertain. American pikas in higher elevation mountain ranges are likely safer from the immediate effects of climate change, but more research is needed to determine their current conservation status, as well as the thermal tolerance of pikas throughout their range. 


\section{CHAPTER 1. FIGURES}

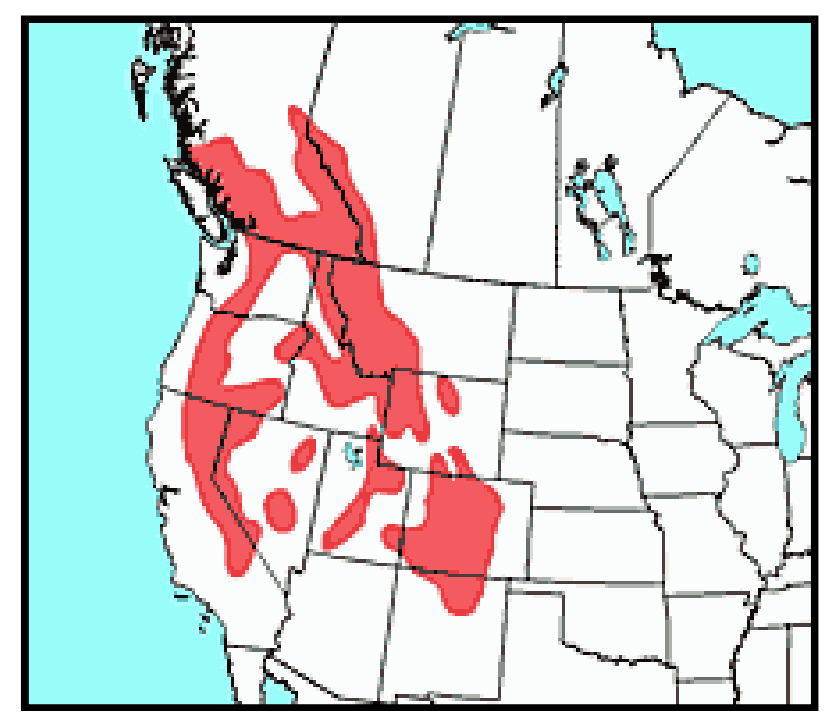

Figure 1. Species range of the American Pika, Ochotona princeps. Map from Smithsonian National Museum of Natural History. 


\section{CHAPTER 1. LIST OF REFERENCES}

Beever, E.A., P.F. Brussard, and J. Berger. 2003. Patterns of extirpation among isolated populations of pikas (Ochotona princeps) in the Great Basin. Journal of Mammalogy 84:37-54.

Beever, E.A., J.L. Wilkening, D.E. McIvor, S.S. Weber, and P.F. Brussard. 2008. American pikas (Ochotona princeps) in northwestern Nevada: a newly discovered population at a low-elevation site. Western North American Naturalist 68:8-14.

Beever, E.A., C. Ray, P.W. Mote, J.L. Wilkening. 2010. Testing alternative methods of climate-mediated extirpations. Ecological Society of America 20:164-178.

Beniston, M. 2003. Climatic change in mountain regions: a review of possible impacts. Climatic Change 59:5-31.

Burger, R. and M. Lynch. 1995. Evolution and extinction in a changing environment: a quantitative-genetic analysis. Evolution 49:151-163.

Conner, D. 1982. Geographic variation in short calls of pika (Ochotona princeps). Journal of Mammalogy 63:48-52.

Conner, D. 1983. Seasonal changes in activity patterns and the adaptive value of haying in pikas (Ochotona princeps). Canadian Journal of Zoology 61:411-416.

Crawley, M.J. 2007. The R book. John Wiley \& Sons Ltd., West Sussex, England.

Dearing, M.D. 1995. Factors governing diet selection in a herbivorous mammal, the North American Pika, Ochotona princeps, Dissertation. University of Utah, Salt Lake City, Utah, USA.

Dearing, M.D. 1997. The function of haypiles of pikas (Ochotona princeps). Journal of Mammalogy 78:1156-1163.

Franken, R.J. and D.S. Hik. 2004. Influence of habitat quality, patch size and connectivity on colonization and extinction dynamics of collared pikas Ochotona collaris. Journal of Animal Ecology 73:889-896.

Grayson, D.K. 2005. A brief history of Great Basin pikas. Journal of Biogeography $32: 2103-2111$. 
Gustafson, J.R. and R.J. Logsdon. 2007 (Dec 21) Evaluation of petition from Center of Biological Diversity to list American Pika (Ochotona princeps) as threatened California Department of Fish and Game, Wildlife Branch, Sacramento, CA. Nongame Wildlife Program Report 2007-05, 19 pp.

http://www.dfg.ca.gov/wildlife/species/publications/docs/PikaEvaluationReportDec2107. pdf

Hafner, D.J. 1993. North American pika (Ochotona princeps) as a late Quaternary biogeographic indicator species. Quaternary Research 39:373-380.

Hafner, D.J. and A.T. Smith. 2010. Revisions of the subspecies of the American pika, Ochotona princeps (Lagomorpha: Ochotonidae). Journal of Mammalogy 91:401-417.

Hayes, A.R. and N.J. Huntly. 2005. Effects of wind on the behavior and call transmission of pikas (Ochotona princeps). Journal of Mammalogy 86:974-981.

Holmes, W.G. 1991. Predator risk affects foraging behavior of pikas: observation and experimental evidence. Animal Behaviour: 42:111-119.

Hosmer, D.W. and S. Lemeshow. 2000. Applied logistic regression, $2^{\text {nd }}$ eds. New York, NY: John Wiley and Sons, Inc.

Huntly, N.J., A.T. Smith, and B.L. Ivins. 1986. Foraging behavior of the pika (Ochotona princeps) with comparisons of grazing versus haying. Journal of Mammalogy 67:139148.

Intergovernmental Panel on Climate Change (IPCC), 2007. Climate change impacts, adaptation, and vulnerability. In: Contribution of Working Group II to the Fourth Assessment Report of the IPCC. Cambridge University Press, Cambridge, UK.

Ivins, B.L. and A.T. Smith. 1983. Responses of pikas (Ochotona princeps, Lagomorpha) to naturally occurring terrestrial predators. Behavioral Ecology and Sociobiology 13:277285.

Jameson, E.W. and H.J. Peeters. 2004. Mammals of California, Revised Edition.

Katzner, T.E., K.L. Parker, and H.H. Harlow. 1997. Metabolism and thermal response in winter-acclimatized pygmy rabbits (Brachylagus idahoensis). Journal of Mammalogy 78:1053-1062.

Kawamichi, T. 1976. Hay territory and dominance rank of pikas (Ochotona princeps). Journal of Mammalogy 57:133-148.

Kosaka, M., G.J. Yang, T. Matsumoto, N. Ohwatari, K. Tsuchiya, C.M. Chen, K. Nakamura, S. Matsuo, and T. Moriuchi. 1988. Analysis of locomotor activity on pika (whistle rabbit). Tropical Medicine 30:213-218. 
Krear, H.R. 1965. An ecological and ethological study of the pika (Ochotona princeps saxatilis bangs) in the front range of Colorado. Ph.D. Thesis. University of Colorado, Boulder.

Kreuzer, M.P. and N.J. Huntly. 2003. Habitat specific demography: evidence for sourcesink population structure in a mammal, the pika. Oecologia 134:343-349.

Laws, J.M. 2007. The Laws Field Guide to the Sierra Nevada. Heyday Books, Berkeley, California.

Loarie, S.R., J.S. Clark, C. Ray, E. Beever, P.B. Duffy, K. Hayhoe, J.L. Wilkening, C.B. Field. 2009. American pika persistence through changing climates. National Academy of Sciences.

Lutton, L.M. 1975. Notes on the territorial behavior and response to predators of the pika, Ochotona princeps. Journal of Mammalogy 56:231-234.

MacArthur, R.A. and L.C.H. Wang. 1973. Physiology of thermoregulation in the pika, Ochotona princeps. Canadian Journal of Zoology 51:11-16.

MacArthur, R.A. and L.C.H. Wang. 1974. Behavioral thermoregulation in the pika, Ochotona princeps: a field study using radiotelemetry. Canadian Journal of Zoology 52:353-358.

McDonald, K.A. and J.H. Brown. 1992. Using montane mammals to model extinctions due to global change. Conservation Biology 6:409-415.

McKechnie, A.M., A.T. Smith, and M.M Peacock. 1994. Kleptoparasitism in pikas (Ochotona princeps): theft of hay. Journal of Mammalogy 75:488-491.

Millar, C. USDA Forest Service. Pika Survey Instruction form (online). Accessed Jan. 3, 2011 at http://www.fs.fed.us/psw/publications/millar/PikaSurvey_Instructions_Form.pdf

Millar, C.I. and R.D. Westfall. 2010. Distribution and climatic relationships of the American pika (Ochotona princeps) in the Sierra Nevada and western Great Basin, USA; periglacial landforms as refugia in warming climates. Arctic, Antarctic, and Alpine Research 42:76-88.

Millar, C. USDA Forest Service. Pika Survey Instruction form (online). Accessed Jan. 3, 2011 at http://www.fs.fed.us/psw/publications/millar/PikaSurvey_Instructions_Form.pdf

Millar, J.S. and F.C. Zwickel. 1972. Characteristics and ecological significance of hay piles of pikas. Mammalia 36: 657-667.

Moilanen, A., A.T. Smith and I. Hanski. 1998. Long-term dynamics in a metapopulation of the American pika. The American Naturalist 152:530-542. 
Moritz, C. 2007. Final Report: A re-survey of the historic Grinnell-Storer vertebrate transect in Yosemite National Park, California. Sierra Nevada Network Inventory \& Monitoring Program, Sequoia \& Kings Canyon National Parks, 47050 Generals Highway, Three Rivers, CA 93271. http://mvz.berkeley.edu/Grinnell/pdf/2007_ Yosemite_report.pdf

Morrison, S., L. Barton, P. Caputa, and D.S. Hik. 2004. Forage selection by collared pikas, Ochotona collaris, under varying degress of predation risk. Canadian Journal of Zoology 82:533-540.

Nichols, L.B. 2010. Fecal pellets in American pikas (Ochotona princeps) provide a crude chronometer for dating patch occupancy. Western North American Naturalist 70:500507.

Peacock, M.M. 1997. Determining natal dispersal patterns in a population of North American pikas (Ochotona princeps) using direct mark-resight and indirect genetic methods. Behavioral Ecology 3:340-350.

Peacock, M.M. and Smith, A.T. 1997. The effect of habitat fragmentation on dispersal patterns, mating behavior, and genetic variation in a pika (Ochotona princeps) metapopulation. Oecologia 112:524-533.

Ray, C. 2006. 2006 Project Report: Testing hypothesized links between climate change and the decline of the American pika. MFWP Scientific Collector's Permit: \#1507. http://www.bristlecone.org/PDFs/pikaproject.pdf

Ray, C., Beever, E., 2007. Distribution and abundance of the American Pika (Ochotona princeps) within Lava Beds National Monument. National Park Service Report, 1-57.

Roach, W.J., N. Huntly, and R. Inouye. 2001. Talus fragmentation mitigates the effects of pikas, Ochotona princeps, on high alpine meadows. Oikos 92:315-324.

Rodhouse, T.J., E.A. Beever, L.K Garrett, K.M. Irvine, M.R. Jeffress, M. Munts, and C. Ray. 2010. Distribution of American pikas in a low-elevation lava landscape: conservation implications from the range periphery. Journal of Mammalogy 91:12871299.

Schmid-Holmes, S., L.C. Drickamer, A. Sessions Robinson. L.L. Gillie. 2001. Burrows and burrow-cleaning behavior of house mice (Mus musculus domesticus). American Midland Naturalist 146:53-62.

Severaid, J.H. 1955. Natural history of the pikas (Mammalian genus Ochotona). Ph.D. Thesis. University of California, Berkeley. 
Shardlow, M. R., J. Apel, L.K. Garrett, G. Holm, D. Larson, N. Nordensten, and T. J. Rodhouse. 2009. Upper Columbia Basin Network American pika monitoring protocol: Narrative Version 1.0. Natural Resource Report NPS/UCBN/NRR-2009/XXX. National Park Service, Fort Collins, Colorado.

Smith, A.T. 1974a. The distribution and dispersal of pikas: consequences of insular population structure. Ecology 55:1112-1119.

Smith, A.T. 1974b. The distribution and dispersal of pikas: influences of behavior and climate. Ecology 55:1368-1376.

Smith, A.T. 1978. Comparative demography of pikas (Ochotona): effect of spatial and temporal age-specific mortality. Ecology 59:133-139.

Smith, A.T. 2008. World of pikas. In Lagomorph Biology Evolution, Ecology, and Conservation, edited by P.C. Alves et al., Springer Berlin Heidelberg. Pp. 89-102.

Smith, A.T. and B.L. Ivins. 1983. Colonization in a pika population: dispersal vs philopatry. Behav Ecol Sociobiol 13:37-47.

Smith, A.T. and M.L. Weston. 1990. Mammalian Species, Ochotona princeps. Journal of Mammalogy 352:1-8.

Smithsonian National Museum of Natural History. North American Mammals: Ochotona princeps (online). Accessed April 20, 2008 at http://www.mnh.si.edu/mna/image_info. cfm?species_id=225

Svendsen, G.E. 1979. Territoriality and behavior in a population of pikas (Ochotona princeps). Journal of Mammalogy 60:324-330.

Trefry, S.A. and D.S. Hik. 2010. Variations in pika (Ochotona collaris, O. princeps) vocalizations within and between populations. Ecography 33:784-795.

Tyser, R.W. 1980. Use of substrate for surveillance behaviors in a community of talus slope mammals. American Midland Naturalist 104:32-38.

United States Fish and Wildlife Service. Department of the Interior Federal Register Notice. February 25, 2010. 12-month finding on a petition to list the American pika as threatened or endangered. http://www.fws.gov/mountain-prairie/species/mammals/ americanpika/

Verts, B.J. and L.N. Carraway. 1998. Land mammals of Oregon. University of California Press, Berkeley. 
Whitworth, M.R. and C.H. Southwick. 1984. Sex differences in the ontogeny of social behavior in pikas: possible relationships to dispersal and territoriality. Behavioral Ecology and Sociobiology 15:175-182.

Wilkening, J.L., C. Ray, E.A. Beever, and P.F. Brussard. 2011. Modeling contemporary range retraction in Great Basin pikas (Ochotona princeps) using data on microclimate and microhabitat. Quaternary International 235:77-88.

Wolf, S., B. Nowicki, and K. Siegel. 2007a. Before the California Fish and Game Commission: Petition to list the American pika (Ochotona princeps) as threatened under the California Endangered Species Act. Center for Biological Diversity.

Wolf, S., B. Nowicki, and K. Siegel. 2007b. Before the Secretary of the Interior: Petition to list the American pika (Ochotona princeps) as threatened or endangered under the United States Endangered Species Act. Center for Biological Diversity. 


\title{
CHAPTER 2.
}

\section{$\underline{\text { Habitat and Temperature Profiles of Historic and Current Pika }}$}

\author{
$\underline{\text { Sites in the Lassen Peak Region }}$
}

\section{INTRODUCTION}

The earth is expected to warm $1-8^{\circ} \mathrm{C}$ in the next century (A1B:SRES scenario, IPCC 2007). This will likely have profound effects on organisms around the world. In the last 100 years the earth has warmed $0.6^{\circ} \mathrm{C}$, and this is already having a measurable effect on many organisms (Walther et al. 2002). Increasing amounts of evidence suggest the phenology of animal and plant activity is being influenced by climate change. Spring activities, such as the breeding of birds and amphibians and the flowering of plants, have occurred earlier and earlier over the last 50 years. As temperatures increase, some animals are moving northward (in the northern hemisphere) and montane animals are moving upward in elevation (IPCC 2007, Parmesan and Yohe 2003).

Montane organisms are at particular risk, not only because of the possible reduction in habitat as optimal conditions rise in elevation, but also because temperatures are predicted to rise more in alpine environments (Beniston 2003, Naftz et al. 2002). At lower elevations in montane areas, losses of species, many of them alpine obligate, have been reported (Lenoir et al. 2008, Walther et al. 2005, Wilson et al. 2005). As snowpack levels decline, and spring snowmelt occurs earlier (IPCC 2007), the community composition of alpine ecosystems could be severely altered. The timing of spring snowmelt determines the flowering time and seed production of many alpine plants 
(Inouye and McGuire 1991), as well as the reproductive output of some alpine mammals (e.g., yellow-bellied marmots, Marmota flaviventris; Van Vuren and Armitage 1991).

Despite the growing body of evidence of the impact of climate change on natural systems, few researchers have identified climate change as a primary cause of specific population extirpations (Beever et al. 2010, McLaughlin et al. 2002). To date, only one species has been granted threatened status through the Endangered Species Act because of climate change, and no species have been listed as endangered. In 2008, the United States Fish and Wildlife Service (USFWS) gave polar bears, Ursus maritimus, threatened status because of habitat loss due to the melting of sea ice (USFWS 2008). Another species that has been proposed for listing under the Endangered Species Act due to climate change is the American pika, Ochotona princeps.

The American pika is a small lagomorph that lives in mountainous areas in western North America. Pikas usually occur at elevations over $1500 \mathrm{~m}$ and are considered alpine mammals. In the southern part of their range, pikas typically live above $2500 \mathrm{~m}$ (Smith and Weston 1990), but have been reported as low as $1827 \mathrm{~m}$ (Millar and Westfall 2010a). Pikas are generalist herbivores, and eat a variety of grasses, sedges, and leafy vegetation. They do not hibernate during the long montane winter; instead they survive on stores of vegetation, or haypiles, stockpiled during the summer and autumn (Smith and Weston 1990).

The American pika lives in talus slopes or other types of broken rock next to suitable vegetation (Smith and Weston 1990). Because areas of talus or broken rock tend to be patchily distributed over a landscape, they effectively act as islands, separating pika demes from each other. In fact, pikas are considered to have a classic metapopulation 
structure (Franken and Hik 2004, Moilanen et al. 1998). They are reliant on patches of talus, which are usually separated by meadows, and they rarely move more than six meters from the edge of the talus to forage (Franken and Hik 2004, Huntly et al. 1986, Roach et al. 2001). There is limited dispersal between patches: about $25 \%$ of juveniles attempt to disperse from their place of birth (Smith and Weston 1990). However, juveniles can apparently disperse only short distances, likely because they are more susceptible to predation the longer they are away from talus. In fact, distances of over $300 \mathrm{~m}$ act as barriers for dispersing juveniles in lower elevations (Smith 1974a).

However, genetic evidence indicates that pikas may be able to disperse as far as $2 \mathrm{~km}$ in some areas (Peacock 1997). Even so, the farther apart these patches of talus, the less chance there is of dispersal between patches (Franken and Hik 2004, Smith 1974a). This could have serious implications in regard to climate change because if habitat conditions deteriorate, pikas would be unable to relocate long distances to find new territories.

Susceptibility to predation is not the only factor that makes dispersal difficult for pikas. Pikas are extremely intolerant of heat. They rely on talus not only for protection from predators, but perhaps more importantly, for thermal cover. Pikas will die if confined above ground at temperatures as low as $25.5^{\circ}-28^{\circ} \mathrm{C}$ (MacArthur and Wang 1973, Smith 1974b). This inability to regulate body temperature during heat is perhaps the trait that makes pikas acutely vulnerable to climate change. Lower elevation pikas, particularly those in the southern portion of their range, may be at elevated risk. Pikas in these areas that are living in patches of more isolated talus, or in patches that are already at the upper elevational limit of a mountain, are at even greater risk because of their limited dispersal abilities. If temperatures rise too high, it is possible that the 
temperatures within the talus could go above the lethal limit. In addition, higher temperatures could decrease the amount of time pikas can spend foraging, preventing them from gaining sufficient body mass and from collecting enough hay for overwintering (Beever et al. 2010, Wilkening et al. 2011). Rising temperatures may also cause a decrease in snowpack levels (IPCC 2007), and this may have a detrimental effect on pikas. The loss of the insulating snowpack layer exposes pikas to lower temperatures, and may negatively impact their survival (Beever et al. 2010, Ray 2006).

Concerns about the effect of global warming on pikas prompted the Center for Biological Diversity to petition the California Fish and Game Commission and the USFWS, to list pikas as threatened or endangered (Wolf et al. 2007a, b). Both agencies denied the pikas listing, largely due to a lack of data on the current distribution and abundance of these animals throughout their range (Gustafson and Logsdon 2007, USFWS 2010). Many researchers are now studying the distribution and climate relations of pikas throughout western North America. Some researchers are revisiting historic locations to discover if changes have occurred in pika populations over the last century (Beever et al. 2003, Erb et al. 2011, Moritz 2007, J. Stewart and D. Wright unpublished data).

Recent resurveys in the Great Basin indicate that pika populations may be declining in response to rising temperatures (Beever et al. 2010). In the late 1990s Beever et al. (2003) performed a resurvey of the Great Basin, based on surveys from the early 1900s. They discovered that 6 of 25 populations of pikas had gone extinct, and many of the remaining populations had moved to higher elevations. The extirpated populations were located at significantly lower elevations (Beever et al. 2003), with 
average summer temperatures $3.3^{\circ}$ to $4.7^{\circ} \mathrm{C}$ higher than extant sites (Beever et al. 2010). In subsequent sampling, Beever et al. (2010) discovered that 3 more populations had been extirpated between 2000 and 2007. They found that chronic heat stress as well as acute cold stress were the best predictors for extirpation of a site. Chronic heat stress (indicated by average summer temperature) remained higher in extirpated sites, and acute cold stress (indicated by number of days below $-5^{\circ} \mathrm{C}$ ) was $17.2 \%$ higher in abandoned sites. Additionally, extirpated sites contained a lower forb cover than persistence sites (Wilkening et al. 2011).

A few researchers are currently studying pikas in California, but there is still much research to be done in that region. Moritz (2007) reported on a resurvey from Yosemite National Park which found that pika populations had moved up $349 \mathrm{~m}$ in elevation, and that their ranges had decreased in size since the early 1900s. These latter results are debatable, however, because only one historic site had been extirpated, and there remains one low elevation historic site that has yet to be surveyed (J. Perrine, pers. comm.). Millar and Westfall (2010a) opportunistically surveyed sites for pikas in the Sierra Nevada and Great Basin in the summers of 2007 and 2008. They found pikas at elevations ranging from $1827 \mathrm{~m}$ to $3887 \mathrm{~m}$, and in a wider range of habitat types than previously recorded. The lower elevation was the lowest elevation ever documented for pikas in this area of their range, and $8 \%$ of the occupied sites were below the accepted regional lower limit of $2500 \mathrm{~m}$. Their results suggest that pikas in California persist in habitats previously unexpected and understudied, and that more research is needed in this area of their range. 
To increase understanding of the American pika's current distribution, we resurveyed the Lassen Peak region of northern California (Figure 1) based on surveys performed by Joseph Grinnell and his colleagues. In the early 1900s, Grinnell, founder of the Museum of Vertebrate Zoology at U.C. Berkeley, and his colleagues, performed extensive vertebrate surveys in California (Museum of Vertebrate Zoology 2009). From 1924-1929, they surveyed the Lassen Peak region, in an area they termed the Lassen Transect (Figure 1) (Grinnell et al. 1930).

In addition to the historic resurveys, we opportunistically surveyed other sites of likely pika habitat in the region. While the Great Basin has been well surveyed for pikas, this area just west of the Great Basin has been largely ignored to date (Figure 2). The Lassen Peak region contains a variety of elevations and habitat types, including alpine and sub-alpine meadows, shrub-steppe, fir, and pine forests. This environmental diversity makes it an important area to survey for pikas because habitat that is considered atypical of pikas (as in, not alpine) is understudied but may contain pikas (Millar and Westfall 2010a). During a time of climate change, it is likely that pikas living in "borderline" habitat, such as dry low elevation sites, will be the first to disappear (Beever et al. 2010). The Lassen Peak region, particularly east of Lassen Volcanic National Park, contains a large amount of borderline habitat. The climate becomes significantly warmer and drier heading east from the national park toward the Modoc Plateau. This type of warm, dry habitat needs to be monitored, because it is likely to show the effects of climate change earlier than high elevation mountain ranges, such as the Sierra Nevada. Monitoring the Lassen Peak region adds valuable insight into the status of American pika populations throughout their range. 
As well as determining which sites were still occupied, we explored microclimatic and microhabitat factors that may affect occupancy. In resurveys of the Great Basin, both climatic and forage-related variables were significant predictors of pika occupancy (Beever et al. 2010, Wilkening et al. 2011). Factors that were investigated in the Great Basin included chronic heat stress, acute heat and cold stress, and relative vegetative covers of forbs and graminoids. In Great Basin studies, chronic heat stress was represented by the average summer temperature, and acute cold and heat stresses were measured by the number of days above (heat stress) and below (cold stress) certain temperature thresholds (heat: $>28^{\circ} \mathrm{C}$, cold: $<0^{\circ}$ and $<-5^{\circ}$ in Beever et al. 2010; heat: $>28^{\circ} \mathrm{C}$ and $>26^{\circ} \mathrm{C}$, cold: $<-5^{\circ}$ and $<-10^{\circ} \mathrm{C}$ in Wilkening et al. 2011). Based on their results, we expected that abandoned sites would have higher average summer temperatures, lower forb cover, and more days above a specific temperature threshold. We explored the significance of acute cold stress, using cold thresholds below specific temperatures, due to a conflict between the results of Beever et al. (2010) and Wilkening et al. (2011), in which acute cold stress was found to be positively associated with site abandonment in the prior, and negatively in the latter. In addition, we investigated chronic cold stress (in the form of the average temperature of the 10 coldest consecutive days), a variable not previously investigated in other resurveys, but one that is worth investigating because pikas appear to be negatively affected by cold temperatures in winter (Beever et al. 2010, Ray 2006). 


\section{METHODS}

\section{Study Area}

We defined the Lassen Peak region as the geographic area that covers the southern end of the Cascade Mountains and extends east into the Great Basin and Madeline Plains (Figures 2 and 3). It includes portions of Shasta, Plumas, Tehama, and Lassen Counties in California. We investigated four main regions, from west to east: Mineral, near the town of Mineral in Lassen National Forest; Lassen Volcanic National Park (hereafter, LVNP); Eagle Lake, Lassen National Forest; and Madeline Plains, in the vicinity of the town of Termo (Figure 3).

The climate becomes warmer and drier heading east from the national park toward the Modoc Plateau. Average annual precipitation levels are as follows, from west to east: Mineral, $140.6 \mathrm{~cm}$; Chester, $85.2 \mathrm{~cm}$; Susanville, $36.3 \mathrm{~cm}$; and Termo, $27.6 \mathrm{~cm}$ (Western Regional Climate Center). The Lassen Peak region contains a diversity of habitat types and elevations, including alpine and sub-alpine meadows, shrub-steppe, fir, and pine forests. In the Mineral and LVNP regions, vegetation is dominated by Douglas fir (Pseudotsuga menziesii) and Jeffrey pine (Pinus jeffreyi). Heading east to Eagle Lake and the Madeline Plains, these larger trees thin out until the dominant vegetation consists of western juniper (Juniperus occidentalis) and sagebrush (Artemisia). We surveyed sites in elevations between 1588 and $2797 \mathrm{~m}$, and covered all aspects and a variety of slopes (from 0 to $42.5^{\circ}$ ). Talus composition varied throughout the sites, and included aa lava as well as dacite. 


\section{Occupancy Surveys}

Using Joseph Grinnell's detailed field notes, my academic advisor, John Perrine, and I resurveyed 17 historic sites in the Lassen Peak region. J. Perrine sorted through the field notes of Grinnell and his colleagues, and identified 17 historic locations. Locations in the notes were sometimes vague, such as "Reading Peak." In these situations, we surveyed all of the appropriate habitat (broken rock or talus fields) within approximately one $\mathrm{km}$ of the area best matching the description. If pikas were found at any site we ceased searching and considered the entire site "occupied". In addition to the historic sites, we opportunistically surveyed 20 sites that we judged to be appropriate pika habitat. We visited these sites in August and September, 2009 and October, 2010. If a site was occupied in either 2009 or 2010 we considered it occupied, regardless of a change in occupancy between the years.

To perform the surveys, we walked parallel lines across slopes, searching for any sign of pikas, particularly pellets and haypiles. We also listened and looked for pikas as we walked. Pika populations are commonly surveyed in this manner, and are highly detectable (>90\%) using these methods (Beever et al. 2003, 2008; Erb et al. 2011; Millar and Westfall 2010a; Rodhouse et al. 2010). Sites were defined as "currently occupied" if they contained sightings, vocalizations, or fresh pellets (greenish in color and stuck to rock sides, see Nichols 2010) (Figure 4). Green vegetation in haypiles was used as a clue to look harder for pellets, but required additional signs to classify the site as occupied because of the risk of confusion with woodrats, Neotoma spp. Woodrats sometimes take over pika haypiles (Millar and Westfall 2010b), and build their own similar stashes of vegetation (Frase and Sera 1993). Sites were considered "abandoned" if they contained 
only pika pellets that were no longer fresh; pellets were white or brown in color, settled on rocks or in latrines, or buried under rocks (see Nichols 2010) (Figure 5). Abandoned sites also sometimes contained old, brown haypiles (stick pieces), and small whitewash spots, but again pellets were the deciding factor to confirm prior pika residence to reduce confusion with other animals.

\section{Temperature Data Collection}

At selected occupied and abandoned sites we installed iButton thermal dataloggers (model DS1922L, Embedded Data Systems, Lawrenceburg, KY) in areas of high current or previous pika use (explanation below). These dataloggers can record 8192 8-bit temperature readings at temperatures ranging from $-40^{\circ} \mathrm{C}$ to $85^{\circ} \mathrm{C}$. To protect the dataloggers, we waterproofed them in Plasti Dip ${ }^{\circledR}$ (Plasti Dip International, Blaine, $\mathrm{MN}$ ) and placed them in copper capsules (Figure 6). We selected copper because of its high thermal conductance, to reduce insulation of the iButton within. We drilled five approximately $7 \mathrm{~mm}$-diameter holes in the capsules to allow for water and air circulation, so that dataloggers could more accurately record the microclimate within the talus. One end of the capsule was screwed on to allow for easy removal of the datalogger upon retrieval of the capsule.

Before deploying the dataloggers, I performed experimental trials with several of the iButtons, exposing them to temperatures ranging from $-5^{\circ} \mathrm{C}$ to $48^{\circ} \mathrm{C}$ using an incubator, refrigerator, and freezer. I compared the equilibration times of dataloggers within a copper capsule and alone to investigate the effect of the capsule on temperature readings. 
For deployment in the field, I programmed the dataloggers to record temperature in Celsius every 1.5 hours and placed them within copper capsules. Each capsule was attached by cable to an aluminum rod that was jammed into the rocks to ensure the capsule was not lost or carried away during the study. The position of each datalogger was recorded with a handheld GPS unit and sometimes marked with flagging to allow for easy relocation the following year.

We placed the capsules approximately one meter in the rocks, in full shade, in areas of high pika use. These high use areas contained elevated concentrations of pika sign, and were usually latrine sites, containing feces and sometimes hay. On occupied slopes, this sign was current; at least some of the feces in the latrine were perched, green pellets, and some of the hay was green (if any). Likewise, on abandoned slopes, all of the sign was old; pellets were settled, and hay was brown (if any).

We installed one datalogger on each slope, except in slopes greater than approximately $150 \mathrm{~m}$ across, in which two dataloggers were installed in different areas of the slope. All dataloggers were at least $100 \mathrm{~m}$ apart, and were placed both high and low on slopes, depending on the location of pika sign. Sites are defined as talus patches that are ecologically distinct from each other, i.e. separated by trees or roads, and that include a change in aspect, elevation, and/or substrate (rock type). All sites were at least $300 \mathrm{~m}$ apart, except in one location on Morgan Mountain in which the sites were $150 \mathrm{~m}$ apart but were separated by a thick band of conifer trees and faced different directions on the mountain (aspects of north, $7^{\circ}$, and east, $99^{\circ}$ ). 


\section{Habitat Surveys}

Datalogger placement marked the center of each habitat survey. Because pikas are central-place foragers (Huntly et al. 1986, Roach et al. 2001), we chose these areas of high use as the center, and did point intercept transects in four directions from the center. To capture differences caused by slope, one transect line ran vertically (uphill), and the other ran perpendicular to the hill, horizontally across the side slope. Each transect line was $25 \mathrm{~m}$ long (total length across), crossing at the location of datalogger placement. A plot $25 \mathrm{~m}$ in diameter has an area of approximately $490 \mathrm{~m}^{2}$, comparable to the territory sizes that have been estimated for pikas (400-700 $\mathrm{m}^{2}$, Smith and Weston 1990). Rodhouse et al. (2010), using the survey protocol designed by Shardlow et al. (2009), employed a $12 \mathrm{~m}$ radius plot, both in surveying for pikas and in studying pika habitat characteristics. We used a similar radius of $12.5 \mathrm{~m}$.

On each transect line, we recorded substrate (rock, dirt, or litter) and vegetation type (forb, graminoid, fern, shrub, or tree) at every meter mark (see Table 1 for a description of habitat parameters). On each of the four arms of the transect, we also measured and recorded the deepest crevice in the rocks using a meter stick (within one meter of the line), and recorded signs of woodrat presence, including whitewash and feces. Substrate, vegetation, depth, and woodrat sign at the center point were only recorded once to prevent duplication.

\section{Data Analysis}

I used logistic regression to model pika persistence as predicted by biologically relevant variables. I chose to investigate predictor variables based on their relevance in 
previous literature (Beever et al. 2010, MacArthur and Wang 1973, Smith 1974b, Wilkening et al. 2011), as well as trends noticed during surveys and preliminary data analysis (e.g. temperature profiles). I grouped predictor variables into seven categories, which were investigated in multivariate logistic regressions: "Elevation", "Substrate", "Vegetation", "Chronic heat stress", “Acute heat stress", "Chronic cold stress", and "Acute cold stress". The first four represent habitat, and the latter four represent microclimate (Tables 1,2). An eighth category, "Descriptive", was not investigated in multivariate regressions.

Descriptions of the habitat variables investigated are listed in Table 1. We investigated elevation of datalogger sites (a macrohabitat variable) to determine if occupied sites were at a higher average elevation than abandoned sites, as found by Beever et al. (2003) and Rodhouse et al. (2010). The microhabitat variables we investigated were the relative covers of vegetation, (forbs, graminoids, shrubs, and trees) as well as the proportion of each substrate (rock, dirt, and litter), the talus depth, and the presence of woodrats. Relative cover was calculated by dividing the cover of a specific vegetation type by the total cover of all plants on the line. The proportion of each substrate type was calculated based on all four transect arms (49 points). We investigated the maximum talus depth (hereafter, "depth") measured in each transect area in an attempt to describe talus structure. At each site, maximum depth was averaged for all transect lines. For depths greater than $1 \mathrm{~m}$ (the limit of the meter stick), the depth was given a value of $1.5 \mathrm{~m}$. It is likely that depth in many places was as deep as $3 \mathrm{~m}$, so a value of $1.5 \mathrm{~m}$ may represent an average depth in deeper talus. Depth of a talus field is an extremely difficult characteristic to quantify, and researchers have tried a variety of 
methods to measure it (Erb et al. 2011, Ray and Beever 2007, Wilkening et al. 2011). In addition, if woodrat sign was found anywhere in the site, the site was listed as "woodrats present". Woodrat sign and depth were not used in the multivariate logistic regression models for reasons outlined in the discussion.

Descriptions of the microclimate variables calculated from the thermal dataloggers are given in Table 2. Several of these variables were chosen due to their significance in predicting pika occupancy in previous studies. For instance, we investigated average summer temperature because Wilkening et al. (2011) found that average summer temperature was higher in abandoned than in occupied sites. We calculated average summer temperature based on the dates of the summer solstice to the fall equinox in both years (date of specific datalogger placement to September 222009 , and June 21 to September 23 2010). In addition to the summer average (hereafter, "summer"), a chronic thermal stress, we investigated three temperature thresholds representing acute thermal stress: the number of days above $20^{\circ} \mathrm{C}$, below $0^{\circ} \mathrm{C}$ and below $-5^{\circ} \mathrm{C}$. Wilkening et al. (2011) and Beever et al. (2010) found the lower thresholds to have varying levels of significance; we included them to shed more light on their relevance in predicting pika persistence in the Lassen Peak region. Wilkening et al. (2011) and Beever et al. (2010) used $26^{\circ} \mathrm{C}$ and $28^{\circ} \mathrm{C}$ as their upper thermal thresholds. We had few sites (five) with values above $26^{\circ} \mathrm{C}$, so we selected an upper thermal threshold of $20^{\circ} \mathrm{C}$ to compare between sites. (Of these five sites with values above $26^{\circ}$ C, two were occupied and three were abandoned.)

We also calculated several microclimate variables that were not investigated in previous studies. These were the total average temperature of the full study (hereafter, 
"mean"), the coefficient of variation (CV) of the mean temperature, the mode, and the averages of the hottest and coldest ten consecutive days (hereafter, "coldest 10" and "hottest 10"). The hottest and coldest 10 days describe chronic thermal stress in each location. The $\mathrm{CV}$ describes the relative amount of fluctuation in temperature at a site. The mode represents a type of chronic stress, because in all cases it represented the temperature during the winter when dataloggers maintained a relatively constant temperature, presumably due to insulation under snow (Figures 11-13).

All values for dataloggers and transects in the same site were averaged (both habitat and climatic characteristics). Temperature varies throughout a talus field; downslope talus tends to be colder than upslope talus (Krear 1965, C. Millar and R. Westfall unpublished data). Because all of our dataloggers were placed in areas of high pika use (current or abandoned), they represent the range of temperatures that a pika would experience, and averaging within sites produces one value per site.

Many of the habitat and climate variables were transformed before analyses to reduce skew in the data. Six habitat variables were log-transformed: the proportions of rock, litter, dirt, and relative covers of forbs, graminoids, and shrubs. Relative tree cover was transformed with a square root function. Two microclimate variables were logtransformed: the number of days below $-5^{\circ} \mathrm{C}$, and the number of days above $20^{\circ} \mathrm{C}$.

I used logistic regression to model pika persistence as predicted by habitat and microclimate variables. Before running the logistic regressions, I checked for correlations between variables using Pearson product-moment correlation matrices and selected less related variables to include in multivariate logistic regressions (using a cutoff threshold of $r \geq|0.70|$, Hosmer and Lemeshow 2000) (Tables 3 and 4). All 
variables were tested in univariate logistic regressions, and less correlated $(\mathrm{r}<|0.70|)$ variables were grouped into categories (listed previously) and tested in additive multivariate logistic regressions. In cases of correlated variables, I selected the variable with higher explanatory performance (based on the log-likelihood values in univariate models). Logistic regressions and other analyses were conducted in R 2.12.0 (@2010 The R Foundation for Statistical Computing, http://www.r-project.org/foundation/). I used an information-theoretic framework to select models (Burnham and Anderson 2002). To rank candidate models, I used $\mathrm{AIC}_{c}$, an information criterion adjusted for small sample sizes, and considered models with $\Delta \mathrm{AIC}_{\mathrm{c}} \leq 2$ to be more supported (Burnham and Anderson 2002). I ran additive multivariate logistic regression models of microclimate and habitat categories separately, then combined categories from the best supported models to produce final models. Analyzing habitat and climate categories separately before combining them allowed me to reduce the number of variables investigated in one model, thus lowering the chance of a Type I error. Analyzing by category, rather than by variable, allowed me to determine the relative importance of each category type (e.g., vegetation) in predicting pika occupancy. In addition to the logistic regressions, I used Welch's two-sample $t$-tests (used for samples with unequal variances) with significance level $\alpha \leq 0.1$ to characterize differences between mean values of certain habitat and microclimate variables in occupied and abandoned sites. Prior to these analyses, several variables were log- and square root-transformed, as described previously. 


\section{RESULTS}

\section{Datalogger Trials}

In trials comparing individual dataloggers, temperature differences were slight (approximately $0.5^{\circ} \mathrm{C}$ different, when set the same and exposed to the same temperature). There was a small difference in equilibration time between dataloggers within capsules, and free dataloggers (Figures 7,8). The encapsulated dataloggers took slightly longer (about $10 \mathrm{~min}$.) to adjust to the ambient temperature. During large temperature changes (of about $23^{\circ} \mathrm{C}$ ), the encapsulated datalogger lagged behind the free datalogger by about 20 minutes, and the largest difference between the two was $6.5^{\circ} \mathrm{C}$.

\section{Occupancy Surveys}

We surveyed a total of 37 sites (17 historic and 20 additional) in the summer of 2009, and resurveyed the 29 sites with dataloggers in the fall of 2010 when we retrieved them (Figure 9, Table 5). At four sites we found no definitive sign of occupancy. We considered one abandoned because it was a historic site, but we did not include the other three sites in the analysis because we do not know if they were ever occupied. The historic site (3 miles northeast of Horse Lake) was well described in the historic field notes, and we were confident that we were in the correct location. Furthermore, the historic site contained indirect evidence of previous pika occupation, in the form of small urine stains (smaller than the stains typically left by woodrats), which were located in a fashion indicative of pikas (Millar and Westfall 2010a). 
In our second surveys of the 29 datalogger sites in 2010 we found changes in occupancy in three sites (Table 5). We found fresh pika sign (scat and clipped vegetation) in one site that had previously been marked as abandoned (Emerald Lake, LVNP). Additionally, we were not able to find fresh pika sign in two other nearby sites that had been occupied the previous year (Eagle Peak and Lassen Peak Trailhead, Table 5). The latter sites retained their denotation of occupied, and the Emerald Lake sign was also denoted as occupied, because all three sites were occupied at some point during surveys.

Of the 17 historic sites, 11 were occupied and 6 were abandoned $(64.7 \%$ occupancy), and of the 17 additional occupancy-known sites, 6 were occupied and 11 were abandoned (35.3\% occupancy) (Table 6). In total, for all sites in which occupancy status was known (i.e. pika sign was found or historic field notes described prior pika presence), 17 sites were occupied and 17 were abandoned.

\section{Datalogger Deployment}

The dataloggers were deployed between August 10 and September 4, 2009, and removed in mid-October, 2010, resulting in 13 to 14 months of temperatures recorded. We deployed a total of 37 dataloggers at 29 distinct sites (14 historic, 15 additional) (Figure 9). The habitat and climatic analyses are based on 29 sites with dataloggers. Due to the variation in talus structure, not all dataloggers could be placed at the same depth, but most were placed between 90 and $100 \mathrm{~cm}$ into the rocks, and all were in complete shade. One datalogger was as shallow as $60 \mathrm{~cm}$. A total of 17 datalogger sites were occupied and 12 were abandoned (Figure 9). 
All but one datalogger site contained definite evidence of current or prior pika occupancy (indirect sign, primarily fresh or old scat). In the one historic site that did not have definitive pika sign, we placed the probe in a location that was most similar to other areas that pikas prefer to use. This location also had a high concentration of small whitewash patches that may have resulted from former pika occupants (Millar and Westfall 2010a).

\section{Pika Sign}

Once on slope, the time needed to locate the first pika sign ranged from 2 to 135 person-minutes in abandoned sites (mean $\pm \mathrm{SE}=23.7 \pm 5.5 \mathrm{~min}$. , median $=15 \mathrm{~min}$.), and 2 to 45 person-minutes in occupied sites (mean $\pm \mathrm{SE}=11.9 \pm 1.5$ min., median $=13$ min.). In occupied sites, the first sign discovered was pellets $90 \%$ of the time, calls $5 \%$ of the time, and sightings and haypiles each $2.5 \%$ of the time (Table 7). For all sites, haypiles (old or fresh) were found $57.1 \%$ of the time (Table 7). Fresh haypiles were discovered $47.4 \%$ of the time in occupied sites.

Pikas were not heard or seen in every site that was considered currently occupied; in $27.3 \%$ of surveys listed as occupied, no calls were heard or pikas seen. These sites were considered occupied based on the presence of fresh pellets. $100 \%$ of surveys of sites that contained direct evidence (calls or sightings) also had fresh pellets. Also, every survey with sightings had calls heard, but not vice versa; at occupied sites, pikas were heard $57.9 \%$ of the time and seen $14.8 \%$ of the time (Table 7 ). 


\section{Habitat Characteristics}

The average habitat characteristics for occupied and abandoned sites are listed in Table 8. The average elevation of abandoned sites (1968 m) was significantly lower than the average elevation of occupied sites $(2178 \mathrm{~m})\left(\mathrm{t}_{23}=-1.98, P=0.06\right)$. The average $( \pm$ SE) talus depth in abandoned sites $(85.4 \pm 9.0 \mathrm{~cm})$ was not significantly different than the average depth in occupied sites $(98.8 \pm 5.6 \mathrm{~cm})$ (Table 8$)$. Woodrat sign was found in $100 \%$ of abandoned sites, and in $47.1 \%$ of occupied sites, and significantly more abandoned sites contained woodrat sign than occupied sites in the two-sample $t$-test $\left(\mathrm{t}_{16}=\right.$ 4.24, $P=0.00)$.

The proportion of litter and the relative covers of graminoids, forbs, and shrubs were all significantly different between occupied and abandoned sites (Table 8). The proportion of litter was significantly higher in abandoned sites $(0.13 \pm 0.004)$ than occupied sites $(0.03 \pm 0.001)\left(\mathrm{t}_{12}=2.69, P=0.02\right)$. The relative covers of graminoids and forbs were significantly lower in abandoned sites than occupied sites (graminoids: $\mathrm{t}_{22}=-2.15, P=0.04$; forbs: $\left.\mathrm{t}_{27}=-2.06, P=0.05\right)$ (Figure 10$)$. The relative cover of shrubs was significantly higher in abandoned sites $(0.53)$ than occupied sites $(0.24)\left(\mathrm{t}_{22}=\right.$ $3.08, P=0.01)$, but the relative cover of trees was not significantly different between occupied and abandoned sites $\left(\mathrm{t}_{26}=0.31, P=0.77\right)$, nor were the proportions of rock and dirt substrates (rock: $\mathrm{t}_{19}=-1.45, P=0.17$; dirt: $\left.\mathrm{t}_{20}=-0.18, P=0.86\right)$ (Table 8 ).

\section{Microclimate Characteristics}

All dataloggers recorded a similarly shaped temperature profile over the course of

the study (Figures 11-13). The highest temperatures occurred around the date of 
deployment in 2009 (mid-August to mid-September), and in July or August 2010. Maximum temperatures ranged from $13.2^{\circ}$ to $28.1^{\circ} \mathrm{C}$ in abandoned sites and from $12.1^{\circ}$ to $36.2^{\circ}$ in occupied sites. Lowest temperatures were typically recorded in late or midfall of 2009, when the dataloggers recorded a sharp decline in temperature. The minimum temperatures ranged from $-12.5^{\circ}$ to $-2.4^{\circ} \mathrm{C}$ in abandoned sites, and from $-12.5^{\circ}$ to $-0.4^{\circ} \mathrm{C}$ in occupied sites. The sharp decline in temperature was immediately followed by a relatively steady temperature (frequently a flat line) at or near freezing, which usually lasted throughout the winter and into the spring. This flat line was the mode, and it varied from $-1.4^{\circ}$ to $2.6^{\circ} \mathrm{C}$ in all sites (abandoned: $0^{\circ}$ to $1.6^{\circ} \mathrm{C}$, occupied: $1.4^{\circ}$ to $2.6^{\circ}$ C). Presumably, this flat line was caused by insulation of snowpack over the rocks, and it was maintained until the snow melted.

The means of climate variables are listed in Table 9. Six predictors were significantly different between occupied and abandoned sites in two-sample $t$-tests: mean, summer, hottest 10 , days below $0^{\circ} \mathrm{C}, \mathrm{CV}$, and days above $20^{\circ} \mathrm{C}$ (Table 9). The total mean was $2.06^{\circ} \mathrm{C}$ higher in abandoned than occupied sites $\left(\mathrm{t}_{24}=2.55, P=0.02\right)$; summer mean was $4.06^{\circ} \mathrm{C}$ higher $\left(\mathrm{t}_{25}=2.61, P=0.02\right)$; and the average of the hottest 10 days was $3.36^{\circ} \mathrm{C}$ higher in abandoned than occupied sites $\left(\mathrm{t}_{21}=2.26, P=0.03\right)$ (Figure 14). Occupied sites had an average of 40 more days below $0^{\circ} \mathrm{C}\left(\mathrm{t}_{25}=-2.08, \mathrm{P}=0.05\right)$, and 30 less days above $20^{\circ} \mathrm{C}$ than abandoned sites $\left(\mathrm{t}_{20}=1.96, P=0.07\right)$. The $\mathrm{CV}$ was significantly higher in occupied than abandoned sites $\left(\mathrm{t}_{19}=-2.36, P=0.03\right)$. The average minimum, maximum, mode, coldest 10 , and the average number of days below $-5^{\circ} \mathrm{C}$ were not significantly different between abandoned and occupied sites in two-sample $t$ tests (Table 9). 


\section{Logistic Regression Models}

\section{Habitat}

Results of univariate habitat logistic regression models are given in Table 10. Relative cover of shrubs and proportion of litter were negatively associate with occupancy at a site in univariate logistic regressions (shrubs, $P=0.013$; litter, $P=0.028$ ). Relative covers of forbs and graminoids were positively associated with occupancy (forbs, $P=0.087$; graminoids, $P=0.095$ ).

The multivariate habitat model with the lowest $\mathrm{AIC}_{\mathrm{c}}$ value contained the vegetation and substrate categories (Table 11). Substrate alone was comparably supported to this model, $\left(\Delta \mathrm{AIC}_{\mathrm{c}}=0.064\right)$ as was substrate and elevation $\left(\Delta \mathrm{AIC}_{\mathrm{c}}=1.336\right)$. All candidate models were better supported than the null model (intercept only) except the model containing the categories vegetation and elevation (Table 11).

\section{Microclimate}

Results of univariate microclimate logistic regressions are given in Table 12. The best supported univariate models were, in order of $\Delta \mathrm{AIC}_{\mathrm{c}}$ : summer, mean, hottest 10 days, $\log$ of days $>20^{\circ} \mathrm{C}, \mathrm{CV}$, and days $<0$. All measures of chronic heat stress (summer, mean, and hottest 10) had negative relationships with occupancy (mean, $P=$ 0.028; summer, $P=0.028$; hottest $10, P=0.037$ ).

The multivariate microclimate model with the lowest $\mathrm{AIC}_{\mathrm{c}}$ value contained only the acute cold category (Table 13). Adding chronic heat stress produced a model that was similarly supported $\left(\Delta \mathrm{AIC}_{\mathrm{c}}=1.305\right)$. All models were better supported than the null model, except for chronic cold (Table 13). 


\section{Final Models}

I combined the best habitat and microclimate models to produce final candidate models (Table 14). The most parsimonious multivariate model contained substrate and acute cold, $\left(\mathrm{AIC}_{\mathrm{c}}=31.397\right)$ and had the lowest $\mathrm{AIC}_{\mathrm{c}}$ value of any other model tested (Table 14). One other model, vegetation and acute cold, was similarly supported $\left(\Delta \mathrm{AIC}_{\mathrm{c}}\right.$ $=1.604)$ (Table 14). All final models were better supported than the null, except the vegetation and chronic heat model (Table 14).

\section{DISCUSSION}

\section{Methodology}

Researchers debate over the best survey method for determining pika site occupation (e.g., participants at the First North American Pika Conference, 27 March 2010, Jackson, Wyoming; http://www.tetonscience.org/index.cfm?id=calendar\&eventID =A349B1BA-1422-0A0A-8C4B3260340EF9ED). There are many proponents of surveying using indirect sign of haypiles and scat (Shardlow et al. 2009; Millar and Westfall 2010a,b; Nichols 2010; Rodhouse et al. 2010), but some argue that direct sign (pika sighting or alarm call) is the only sure method of determining occupancy (Wolf 2010, E. Beever pers. comm.). Unfortunately in many regions, pikas are crepuscular (Smith 1974b, Behavior chapter), so surveyors are restricted in the time of day they can survey in order to ensure pika sightings. Furthermore, pikas that exhibit more severe crepuscular behavior tend to live in lower elevation, lesser quality habitat (Smith 1974b, 
Behavior chapter), and pikas living in lesser quality habitat may be "shyer" than those in more typical, higher elevation, habitats (L. Peterson, C. Millar, pers. comm; pers. obs.). Because of the restrictions in time of day to survey, and the difference in pika behavior between different populations, using sign may be a more efficient and accurate method to determine occupancy.

Pikas leave several types of sign, such as haypiles, scat (including large latrines), urine stains (Millar and Westfall 2010a), scattered clipped vegetation, and cropped vegetation near talus borders (Millar and Zwickel 1972). Not all types of sign, however, may be equally useful in determining occupancy. The use of haypiles to determine occupancy is questionable for three reasons. First, we did not find haypiles at every occupied pika site. Not all pikas (both populations and individuals) construct haypiles (Beever et al. 2008, Ray and Beever 2007, pers. obs.). Second, pikas are not the only species to collect vegetation in talus fields. Woodrats (Neotoma spp.) collect vegetation and store it in rocks (Frase and Sera 1993), sometimes in a very similar manner to pikas (Millar and Westfall 2010b). In one location in Flatiron Ridge, we found a very fresh haypile with no new pika pellets, but many fresh rodent pellets that resembled woodrat scat. There was a large amount of old pika scat buried underneath the vegetation, supporting the idea that woodrats may take over former pika haypiles (Millar and Westfall 2010b). Finally, using hay as a proxy for occupation would restrict surveys to late summer in many areas. Haypiles are not collected throughout the entire season; the timing of haying depends upon the elevation and the amount of precipitation the previous winter (Smith 1974b). 
Although not all pikas hay, all pikas do defecate, and pika scat can be easily distinguished from the scat of other species (Nichols 2010). Pikas produce two types of fecal material: hard round pellets, and soft shiny caecal material that is usually consumed after deposition (Smith and Weston 1990). Severaid (1955) recorded an average of 29 pellets produced per hour by a captive pika. Pikas deposit most of their pellets in latrines, which are obvious and large, further aiding surveyors to find pika sign. In our surveys, the first sign we discovered was scat $94 \%$ of the time. In occupied areas we found scat, on average, within 12 person-minutes of arrival on a slope, although usually we found scat within five minutes of arrival. Scat is not only easy to identify and find, but in our study, we found it to be consistent in determining occupancy: in every site that we saw or heard pikas, we found fresh scat. In no instances did we directly detect pikas and not also find fresh pika scat. However, we did visit sites in which no pikas were heard or seen, but fresh scat was found. This is to be expected because many of our surveys took place from late morning to afternoon, when pikas are less active (Smith 1974b, Behavior chapter). It is possible that these areas without direct detections or haypiles were not utilized as much by pikas, and pikas deposited scat while travelling through the area. However, pikas do not typically travel great distances, and have relatively small home range sizes, (861-2182 $\mathrm{m}^{2}$, depending on the estimation methods used; Smith and Weston 1990) so it is more likely that pikas were present somewhere on the slope but inactive at the time of our survey.

In addition to being easily detectable in occupied sites, pika scat can be used to determine historic site occupancy. Pika scat can persist in the environment for decades; a study in Bodie State Historic Park (Mono County, California) showed that pellets can 
persist at least 36 years (Nichols 2010). Using color and consistency of scat, it is possible to determine whether an area has ever been occupied by pikas, and approximately how long the site has been abandoned (Nichols 2010). We found pika scat in all unoccupied historic sites except one, (3 miles northeast of Horse Lake) which is further evidence that scat can persist in the environment after pikas have left. The one historic site in which scat was not found may have been abandoned for such a long time (at least 50 years) that pellets have degraded, or we may simply not have looked hard enough for pellets. In a nearby historic site, above the town of Termo, it took three people 45 minutes (or 2.25 person-hours) to find pellets, and these were found only by excavation of talus. Pellets in that location were settled deep within rock crevices, extremely fragile, gray in color, and much smaller in diameter than other pellets. All of these characteristics are consistent with the oldest pellets (> 12 years) found in the Bodie Hills (Nichols 2010).

To date, scat has been analyzed to determine identifiable aging characteristics in only one geographic area (the Bodie Hills, Nichols 2010). Nichols (2010) showed that pellets in the Bodie Hills have distinctive color, texture, and diameter changes within the first 12 years of deposition (Nichols 2010). Whether these color changes hold true in other regions is currently unknown. Climate likely plays a major role in how long scat remains intact; scat may persist longer in dry environments with cold winters (Nichols 2010). Pika scat also may vary among different populations, depending on the biology of the pikas (such as the size, Nichols 2010) and the vegetation available. Therefore, aging scat from color and texture alone could be risky in other environments.

Some areas of the Lassen transect, particularly in the east near Eagle Lake and Termo, have a similar climate to the Bodie Hills. These areas of the Lassen region 
receive relatively little precipitation and are dominated by sagebrush (Artemisia tridentata). However, as aging pika scat in the Lassen Peak region has never been attempted, we were hesitant to classify pellets as fresh based on color alone. Instead, we used the position of the scat as the final deciding factor on whether or not scat was fresh. To be considered occupied, the pellets had to be "perched", in other words, stuck almost vertically to a rock face, in a way they almost surely would not last through multiple seasons. Most likely, the first rainfall or snowmelt would wipe perched pellets off the side of a rock, and cause them to become "settled". Furthermore, sticky pika urine causes pellets to become perched, and this urine loses its stickiness over time (Millar 2011). In all sites in which we found perched pellets, they were greener and more fibrous inside, further supporting the idea that they were fresh. In the Bodie Hills, the green fibrous material within pellets degrades over time resulting in pellets that are brown and more consistently textured inside (Nichols 2010). A useful phrase to remember when surveying for pikas is, "Perched pellets are positive proof of pika presence."

The utility of pellets in estimating pika population size is questionable. Millar and Westfall (2010a) attempted to do this by counting the areas of sign separated by at least $50 \mathrm{~m}$, which exceeds the average nearest-neighbor distance between pikas (Smith and Weston 1990). Unfortunately pika population density seems to be highly variable among different habitat types (C. Millar pers. comm, pers. obs.), so using distance between sign is probably not an accurate censusing method. Trapping or observing pikas is likely the most reliable method of censusing populations. 


\section{Pika Site Occupancy}

Of the 17 historically occupied sites, six (35\%) had been abandoned. The reasons for site abandonment are unknown, but are explored below (in habitat and microclimate sections). Unfortunately we have no record of the timing of abandonment, and sites have likely been abandoned for varying lengths of time. If a universal method was created for aging pellets, we could determine the age of relic pellets in these abandoned sites, and estimate the length of time since site abandonment. Without the timing of abandonment, determining the causes is more difficult.

Some pika sites may merely be abandoned because of natural fluctuations in patch occupancy due to metapopulation dynamics. Patch occupancy in metapopulations is affected by many factors, including size and quality of patch, and distance between patches (Hanski 1994). Metapopulation dynamics confound all short-term studies of pika occupancy, particularly of resurveys that attempt to document changes in occupancy on relatively small spatial scales. We resurveyed all 29 of our habitat and datalogger sites in October 2010 during datalogger removal, and found changes in occupancy in three sites within only one year. One site that had been previously marked as abandoned had become occupied (Emerald Lake, LVNP). Additionally, we were not able to find fresh pika sign in two other nearby sites that had been occupied the previous year (Eagle Peak and vicinity, LVNP). These latter sites retained their denotation of occupied because we believed their abandonment was due to metapopulation dynamics, and not true (or "permanent") extirpation events. Likewise, the Emerald Lake site with new current sign was denoted as occupied in analysis. In order to be sure of true extirpation events, we would need to revisit all sites in multiple years, in a long-term study. Additionally, to be 
sure that populations had not merely relocated over time, it would be beneficial to survey all appropriate pika habitat within at least a $2 \mathrm{~km}$ radius from the historic location $(2 \mathrm{~km}$ is the accepted upper dispersal limit of pikas; Peacock 1997). Due to limitations in time and personnel, the most we could survey was pika habitat within approximately $1 \mathrm{~km}$ of historic locations.

\section{Habitat Relationships}

When interpreting results of the habitat analyses, it is important to remember that our habitat transects were located in areas of high pika use, which tended to be on the edge of the talus field, in varying locations on the slope, but nearly always at or near the talus-vegetation interface. Pikas are central place foragers and thus tend to forage only a short distance from their core territory area (about $2 \mathrm{~m}$ from the talus edge, on average; Huntly et al. 1986, Roach et al. 2001). These habitat transects should therefore represent the available forage, as well as the habitat terrain, most often utilized by pikas. In abandoned sites, we have no way of knowing if the vegetation and habitat terrain have changed since abandonment.

One habitat variable that we recorded but did not use in logistic regressions was the presence of woodrats. We did not use this variable in multivariate modeling because we were not satisfied with the accuracy of our sampling methods for two reasons. First, we recorded woodrat sign, but we could not determine whether the sign was fresh or old. Therefore, in some sites we may have been recording historic woodrat occupancy, rather

than current. Second, we could not confidently differentiate woodrat scat from the scat of similarly-sized rodents such as ground squirrels (Spermophilis spp.). With these caveats 
in mind, our preliminary analysis (see results of $t$-tests) suggests a negative relationship between pikas and woodrats. All of the abandoned sites contained what we believed was woodrat sign, while less than half of the occupied sites did. Without a time scale, or confirmation that the sign was left by woodrats, the relationship is not worth analyzing further at this time. Based on the significance of these preliminary results, however, the relationship between woodrats and pikas is worth exploring in future field studies.

I investigated the types of substrate as well as the talus depth in habitat transects in an effort to quantify the talus infrastructure and to describe infill, if there was any. During pika surveys, it appeared that pikas may prefer more heterogeneous sites (versus sites containing all rocks, for instance). I was hoping that the measures of substrate proportions and depth would quantify heterogeneity in rock slopes. However I chose not to include depth in multivariate models because I was not satisfied with our methodology. The low significance of depth in $t$ tests and univariate regressions is likely a result of insufficient methodology rather than a lack of biological importance. Deeper in talus, temperatures are milder (warmer in winter and cooler in summer) than ambient temperatures (Krear 1965, C. Millar and R. Westfall unpublished data). Rodhouse et al. (2010) speculated that pikas in lava formations can survive higher ambient temperatures due to the greater structural complexity and depth of lava formations (specifically, pahoehoe vs. aa lava). Unfortunately, talus depth is extremely difficult to measure. We used a meter stick, which is much too short to measure maximum depths, and is also straight and non-flexible. Pikas do not need straight tunnels to access the talus matrix; they can weave their way through the rocks in a way we could not measure with a straight instrument. It seems that a better way to quantify talus depth would be through 
the use of some type of SONAR or remote sensing device, to determine the actual depth to the dirt or bedrock. However, even deep talus needs to have interstitial space, and likely a specific size of spacing, to be appropriate habitat for pikas. Measurements of talus depth and spacing would be very useful, but as of yet, as far as we know, no researchers have found a way to measure it.

The substrate proportions of rock and dirt were not significant in univariate models. However, the substrate category was highly supported in all of the best candidate models, including the final models that combined both climate and habitat variables. This is likely due to one variable, litter, which was highly supported in the univariate model. In all models, the proportion of litter was negatively associated with pika presence. On average, the proportion of litter substrate in abandoned sites was more than four times higher than in occupied sites (13\% abandoned, vs. 3\% occupied). The meaning of this is unclear. A higher proportion of litter may indicate infill within rock crevices. Talus with more litter may have less interstitial space, reducing a pika's access to the thermal and safety cover of the rocks. Some of the litter that we recorded was in the form of pine needles, which were packed into rock crevices. A greater proportion of litter could be occurring because there are more shrubs and trees in the abandoned sites. However, neither shrub nor tree cover were highly correlated with litter $(r=0.28-0.33)$, and, furthermore, tree cover was positively (but non-significantly) associated with persistence. Another possibility is that the greater proportion of litter in abandoned sites is a result, rather than a cause, of site abandonment. Perhaps there is more debris on unoccupied slopes because pikas are not there to clean it up. This could be tested in future experiments by using exclosures to keep pikas out of certain areas, or 
observationally by measuring litter substrate in occupied and unoccupied areas. As far as we know this type of "cleanup" behavior has never been recorded in pikas, but they could conceivably remove smaller pieces of litter to keep rock crevices open. Burrow cleaning behavior has been recorded in other small mammals (e.g., house mice, Schmid-Holmes et al. 2001; Richardson's ground squirrels, Quanstrom 1971). In an unrelated study, I did witness a pika moving sticks from the slope into its haypile. The possibility of a cleanup behavior seems unlikely, however, largely for the reason that this behavior has not been recorded by other researchers, and a great number of observational studies have been conducted on pikas. Perhaps changes in vegetation, which could be the result of climate change, have resulted in a greater amount of litter on unoccupied slopes. The relationship between litter and pika occupancy is worth more investigation in future studies.

The vegetation category was highly supported in many of the top-rated models. All vegetation variables except tree cover were significantly different between occupied and abandoned sites. Occupied sites had greater forb and graminoid covers and lower shrub cover, and these parameters were similarly associated with persistence in all models (forb and graminoid cover positively, and shrub cover negatively). Again, vegetative cover differences could be the result of pika abandonment, rather than the cause. Pikas influence plant community composition through foraging and haypile collection (Roach et al. 2001 and Huntly 1987). Roach et al. (2001) and Huntly (1987) found an increase of forb and graminoid covers in pika exclusion plots. Huntly (1987) also recorded an increase in woody plant cover in plots after two years of pika exclusion. If we envision the abandoned sites in our study as glorified exclosure experiments, (of unknown length) we see opposite results in forb and graminoid covers, but similar results 
in shrub (woody plants) cover. It seems unlikely, therefore, that pika abandonment was the primary cause of vegetation changes, at least for forbs and graminoids. However our "experiment" may be on such a different timescale than the experiments described above that it is not comparable.

Wilkening et al. (2011) and Rodhouse et al. (2010) also recorded higher covers of forbs in occupied sites than in abandoned sites. However, researchers in these studies did not find the same relationship in graminoid cover. Rodhouse et al. (2010) actually found a negative relationship between graminoid cover and pika occupancy. Graminoids are less toxic than forbs and shrubs and are believed to be preferred by pikas for immediate consumption (Huntly et al. 1986, Dearing 1997a,b). Forbs and shrubs tend to have higher levels of toxic secondary compounds, causing them to be more difficult for a pika to digest (Dearing 1997a,b; Shipley et al. 2006). When pikas collect toxic vegetation in haypiles, they do not consume it until the secondary compounds in the vegetation have degraded (after a few months) (Dearing 1997a). Because of the high forb content in haypiles (Dearing 1997a), the greater preference for forbs suggests that haypiles may be very important to pikas in the Great Basin (Wilkening et al. 2011) and in Craters of the Moon National Monument (Rodhouse et al. 2010). Additionally, forbs tend to have high moisture content (Dearing 1995), so they may be especially important to pikas in xeric environments, which includes both of the aforementioned regions. In our study region, pikas persist in sites with both more forbs and more graminoids, suggesting both winter (haypiles) and summer forage may be equally important for pikas in this region (assuming they have similar forage preferences to pikas in Colorado, Dearing 1997a). 
Shrub cover has not been investigated in previous pika occupancy studies. Of all vegetative covers, shrub cover was the most different between occupied and abandoned sites, with more than two times the shrub cover in abandoned sites than in occupied sites (Table 8). As shrubs are frequently found in pika haypiles (Huntly et al. 1986, pers. obs.), it is unclear why pikas would prefer sites with lower shrub cover. Perhaps the increase in shrubs is, instead, indicative of a change in the vegetative community and the climate regime of these abandoned sites. Many of the shrubs in our study sites were sagebrush (Artemisia tridentata), which tends to grow in xeric environments. Pika site selection could therefore be based more on precipitation levels than on the vegetation itself, particularly since forbs and graminoids require more mesic environments than sagebrush. Other researchers have also suggested precipitation may be very important for pikas (Beever et al. 2003, 2010; Erb et al. 2011; Wilkening et al. 2011). In fact, pikas have been called indicators of cool, mesic environments (Hafner 1993). Unfortunately, spatially explicit precipitation data are hard to obtain, as well as to forecast (Wilkening et al. 2011). In future analyses, we would like to investigate the importance of water to pikas, perhaps by investigating the distance of our sites to sources of water, such as lakes and streams, and by looking at the presence of water under or at the base of talus, as in Erb et al. 2011 .

The average elevation of occupied pika sites was $210 \mathrm{~m}$ higher than abandoned sites, which supports our hypothesis that abandoned sites would be lower in elevation, and it is also consistent with results of Great Basin resurveys (Beever et al. 2003). Elevation was not highly supported in final multivariate models. This may be partially due to the fact that many regions of the Lassen Peak region contain lava formations, 
which may have important consequences for pikas. Pikas in Lava Beds National Monument in northern California, and Craters of the Moon National Monument in Idaho can survive warmer, drier conditions when they have access to lava formations (Beever 2002, Rodhouse et al. 2010). Beever (2002) and Rodhouse et al. (2010) speculated that lava formations, particularly deep caves, provide cool refugia during times of high temperatures, enabling pikas to live in warmer environments at lower elevations. Because most of their ability to thermoregulate in warm temperatures is behavioral (MacArthur and Wang 1974, Smith 1974b), cool refugia are of key importance to pika survival. Given that pikas in the Lassen Peak region have access to lava formations, they may persist at warmer climates, and thus lower elevations, than expected at that latitude.

While surveying the Fantastic Lava Beds in LVNP (elevation $1852 \mathrm{~m}$ ), we found direct evidence of the cooling effect of lava formations. In the heat of summer, we discovered ice within the talus matrix. In future surveys, it may be worth investigating talus ice features in all occupied and abandoned pika sites. Perhaps some form of yearround ice or water within the talus is required for pika survival in warmer sites.

\section{Microclimate Relationships}

The best supported univariate microclimate models predicted pika occupancy based on chronic heat stress, all of which were negatively associated with persistence. Just as in the Great Basin (Beever et al. 2010, Wilkening et al. 2011), abandoned sites in the Lassen Peak region had significantly higher mean summer temperatures (about $4^{\circ} \mathrm{C}$ higher in Lassen, on average). Pikas cannot regulate their body temperature to heat physiologically, so they must regulate behaviorally. They tend to be less active and 
remain under talus during higher temperatures (MacArthur and Wang 1974, Smith 1974b). Because of this, higher temperatures could negatively impact pika survival. If pikas are limited in the amount of time they can spend gathering haypiles and foraging due to high temperatures, survival rates and population health could be reduced. Undernourished pikas could be more susceptible to disease and parasites and less reproductively successful. Both reduced summer foraging and lower haypile size could increase pika mortality by reducing a pika's preparedness for winter. In particular, if summer conditions were warm enough to prevent sufficient haying, and winter conditions prevented or reduced access to forage material, pika survival could be severely diminished. It is possible that this may have occurred in the 17 abandoned sites in the Lassen Peak region.

Describing acute heat stress was problematic in our sites. The highs of the Lassen Peak sites were cooler than those in sites in the Great Basin, so the higher thermal thresholds of $26^{\circ} \mathrm{C}$ and $28^{\circ} \mathrm{C}$ used by Wilkening et al. (2011) did not yield significant results. This is more likely due to the warmer climate of the Great Basin than it is to datalogger placement, as our deployment protocols were similar (apart from the deployment site mentioned below). We chose a lower upper thermal threshold of $20^{\circ} \mathrm{C}$, because it more accurately described the spread of maximum temperatures among our sites. Almost half of the sites reached temperatures above $20^{\circ} \mathrm{C}$, while under $14 \%$ of sites recorded temperatures above $26^{\circ} \mathrm{C}$ (with no trend in abandoned vs. occupied sites for the latter).

Another issue in choosing temperature thresholds is determining the scale in which to investigate. We chose to look at the number of days in which specific highs or 
lows were recorded, but we could also have looked at the number of records. The number of 1.5 -hour points that had reached these highs or lows may be more relevant to pikas than the number of days. For instance, conditions in a site with 16 records over $26^{\circ}$ $\mathrm{C}$ in one day could be more hostile to pikas than those in a site that only spiked above $26^{\circ}$ $\mathrm{C}$ once in a day. Yet using the number of days scale, these sites would receive the same weight, for that day. Other researchers using dataloggers have recorded temperatures at varying intervals (from 1-6 hours), so a measure of the number of records of specific temperature thresholds cannot be compared among studies. We chose to investigate the number of days, so that we could compare our results with previous studies (Beever et al. 2010, Wilkening et al. 2011), as well as future studies that are likely to emerge in this very active field of pika biology.

At our sites, extreme highs were not predictive of pika site abandonment. In fact, the two dataloggers that recorded the highest maximum temperatures and the most days above $28^{\circ} \mathrm{C}$ were at occupied sites (Mosquito Pond, LVNP, elevation $2256 \mathrm{~m}$; Papoose Meadows, Eagle Lake, elevation $1627 \mathrm{~m}$ ). The Mosquito Pond datalogger recorded the highest temperatures of all dataloggers $\left(36.2^{\circ} \mathrm{C}\right.$ compared to $33.6^{\circ} \mathrm{C}$ second highest, at Papoose). The difference is more extreme when we compare site averages, because the Mosquito Pond site, being one of the smallest sites, contained only one datalogger. Papoose Meadows contained two dataloggers, so averaging their values reduced the site's maximum temperature to $26.2^{\circ} \mathrm{C}$, which is no longer the second highest recorded site maximum temperature. Deploying multiple dataloggers at each site (preferably more than three) would help to reduce the weight of extreme temperatures recorded, and they would yield a more accurate representation of the talus microclimate at each site. We did 
use multiple dataloggers in sites that were bigger in size. Sites with only one datalogger per site were smaller in size, and we believed would experience a narrower range of temperatures than larger sites in which dataloggers were widely spaced on the slope.

The extreme reading at Mosquito Pond is likely a function of datalogger placement. This location was one of the few sites in which a datalogger was placed directly on a haypile. Millar and Westfall (unpublished data) placed dataloggers on haypile surfaces (shaded) and compared them to temperatures measured within the talus matrix. Temperatures on haypiles tended to be more extreme, particularly in the highs during summer. This is consistent with results of their comparisons between talus surface temperatures and matrix temperatures. We did not place dataloggers on the surface, but some were placed on haypiles, shaded under boulders. Pikas may cache haypiles in warmer areas of talus because they spend more time in these locations in winter, when presumably they are searching out warm temperatures rather than cooler temperatures. The haypile area is not necessarily where the pika goes in the summer to release heat on warm days. Additionally, haypiles may release their own heat as the vegetation within decomposes. The Mosquito Pond deployment site, in addition to being in a haypile, was not within the talus infrastructure as much as other probes. Instead, it was under one large boulder at the edge of the talus field, in a small cave structure rather than actually buried within the narrow talus matrix. It was on flat ground, surrounded by dirt (this location also had the second highest proportion of dirt in habitat transects). While it is typical that many areas of high pika use were located on the edges of talus fields, this site was more extreme in this regard, and more separated from the talus (Figure 15). Interestingly, this datalogger did not record extreme cold temperatures. 
Recently researchers have suggested that severe cold temperatures may be just as important in influencing pika occupancy as severe warm temperatures (Beever et al. 2010, Ray 2006). Pikas may face colder winters because of climate change, due to lower snowpack (IPCC 2007), which results in a lack of insulation on the talus (Beever et al. 2010). Our best models contained two measures of acute cold stress: the number of days below $0^{\circ} \mathrm{C}$, and the minimum temperature recorded. Both of these predictors were positively associated with persistence. In the case of days below $0^{\circ} \mathrm{C}$, this result is contradictory to results of Beever et al. (2010), who found a negative relationship between persistence and days below $0^{\circ} \mathrm{C}$. In our study area, the number of days below $0^{\circ}$ C may not be representative of cold stress for pikas. It may be worthwhile to explore lower thermal thresholds, as Wilkening et al. (2011), who explored a threshold of $-10^{\circ} \mathrm{C}$ (but still found a positive relationship with persistence). In our study, the number of days below $-5^{\circ} \mathrm{C}$ was negatively associated with pika persistence in univariate logistic regression, although this model was not well supported (Table 10).

Another way of exploring acute thermal stress is to use the minimum temperature, as we did. Minimum temperature was less strongly correlated with number of days below $0^{\circ} \mathrm{C}(\mathrm{r}=-0.58)$, than with days below $-5^{\circ} \mathrm{C}(\mathrm{r}=-0.87)$, further suggesting that it may be a better method of exploring acute cold stress than days below $0^{\circ} \mathrm{C}$. In all of the most supported models, minimum temperature was positively associated with persistence; sites with higher minimum temperatures were more likely to be currently occupied by pikas. Therefore, occupied sites were less cold in the extremes, but more moderately cold overall than abandoned sites (with a higher minimum temperature, but more days below $0^{\circ} \mathrm{C}$ ). In other words, pikas did not persist in sites that were both too 
extremely cold and not cold enough (too warm). This is further supported by the negative relationship found in all univariate microclimate models based on both chronic and acute heat stress variables.

Chronic cold stress in the Lassen Peak region was not significant in predicting pika occupancy; the category was poorly supported in all models. Neither the coldest consecutive ten days, nor the mode (the temperature during snowpack in winter) were predictive of pika occupancy or differed significantly between occupied and abandoned sites. Perhaps there is a different way of measuring chronic cold stress that could be predictive of occupancy, or perhaps pikas are so well adapted to cold temperatures (MacArthur and Wang 1973) that extended cold periods are not stressful for them, as long as temperatures are not too extreme and pikas have access to a food source.

\section{Final Remarks}

Results of Lassen surveys, while incomplete, raise concern about current population trends of pikas in the Lassen Peak region; over 35\% of the historic sites were abandoned. While the extirpations noted here cannot be attributed to climate change for certain, the factors that differed between occupied and abandoned sites do suggest that climate change could be having a negative impact. These climate change effects are likely both direct and indirect, and include increased summer temperatures, reduced snowpack, and altered vegetative communities. It will be important to continue to monitor pikas in this region. In addition, a more thorough investigation of these sites is needed. First, in future surveys, the scale of surveys should be enlarged to allow researchers to distinguish population extirpations from natural metapopulation 
fluctuations. Second, to gain a more accurate representation of the talus microclimate, multiple thermodataloggers should be spread throughout each site. Third, analysis of snowpack levels and precipitation, both historic and current, could shed light on reasons behind certain population extirpations. And finally, to confirm our belief that perched pellets are indicative of pika presence, we would like to photograph perched pellets and document their changes over time. In this way we could determine how long pellets stay perched, and refine (or not) survey methods.

Due to the fact that there is not enough information on the overall distribution and abundance of pikas, the Lassen Peak region surveys are extremely valuable. The information we collected can be used for management decisions, and for establishing a baseline for future monitoring as temperatures continue to increase. This information also increases understanding of the significantly understudied habitat and microclimatic preferences of pikas in northern California. A randomized sample of talus and other suitable rock-fields in this region would further increase our knowledge of pika habitat preferences, and by searching for relict fecal pellets we could continue our comparisons of occupied and abandoned sites. 


\section{CHAPTER 2. TABLES}

Table 1. Habitat characteristics investigated, with categorization shown. Values for all sites with $>1$ dataloggers (which were placed at the center of habitat transects) were averaged. The descriptive category was not used in multivariate modeling.

\begin{tabular}{|l|l|l|}
\hline Variable & Category & Explanation \\
\hline Elevation & Elevation & Elevation at the point of datalogger deployment (m) \\
\hline Dirt & Substrate & Proportion of substrate that is dirt \\
\hline Rock & Substrate & Proportion of substrate that is rock \\
\hline Litter & Substrate & $\begin{array}{l}\text { Proportion of substrate that is litter (dead branches, pine needles, and } \\
\text { other biotic debris) }\end{array}$ \\
\hline Forbs & Vegetation & Relative cover of forbs (herbaceous flowering plants, not graminoids) \\
\hline Graminoids & Vegetation & Relative cover of graminoids (grasses, sedges, and rushes) \\
\hline Shrubs & Vegetation & Relative cover of shrubs (woody perennials, not trees) \\
\hline Trees & Vegetation & Relative cover of trees \\
\hline Depth & Descriptive & $\begin{array}{l}\text { Average of maximum depth of talus in the area of datalogger } \\
\text { deployment (cm) }\end{array}$ \\
\hline Woodrat & Descriptive & $\begin{array}{l}\text { (Y/N) If woodrat sign was found anywhere in the site, woodrats were } \\
\text { considered present }\end{array}$ \\
\hline
\end{tabular}

Table 2. Microclimate characteristics investigated, with categorization shown. Values for all sites with duplicate dataloggers were averaged. The descriptive category was not used in multivariate modeling.

\begin{tabular}{|l|l|l|}
\hline Variable & Category & Explanation \\
\hline Mean & Descriptive & $\begin{array}{l}\text { Total average temperature recorded by datalogger over } \\
\text { course of study (C) }\end{array}$ \\
\hline CV & Descriptive & Coefficient of variation of mean temperature \\
\hline Mode & Chronic cold stress & Most frequent temperature recorded (C) \\
\hline Minimum (Min) & Acute cold stress & Lowest temperature recorded over course of study (C) \\
\hline Summer & Acute heat stress & Highest temperature recorded over course of study (C) \\
\hline Hottest 10 & Chronic heat stress & $\begin{array}{l}\text { Average temperature recorded during the calendar days of } \\
\text { summer (summer solstice to fall equinox) for both } 2009 \text { and } \\
\text { 2010, during dates of deployment }(\mathrm{C})\end{array}$ \\
\hline Coldest 10 & Chronic heat stress & $\begin{array}{l}\text { Average temperature of the warmest } 10 \text { consecutive days } \\
\text { during course of study }(\mathrm{C})\end{array}$ \\
\hline Days $<0$ & Acute cold stress & $\begin{array}{l}\text { Average temperature of the coldest } 10 \text { consecutive days } \\
\text { during the course of study (C) }\end{array}$ \\
\hline Days $<-5$ & $\begin{array}{l}\text { Number of days in which dataloggers recorded } \\
\text { temperature(s) below } 0^{\circ} \mathrm{C}\end{array}$ \\
\hline Days $>20$ & Acute cold stress & $\begin{array}{l}\text { Number of days in which dataloggers recorded } \\
\text { temperature(s) below }-5^{\circ} \mathrm{C}\end{array}$ \\
\hline
\end{tabular}


Table 3. Correlation matrix of habitat variables, using Pearson product-moment correlation coefficient $(\mathrm{r})$. None of the habitat variables were highly correlated $(\mathrm{r}<$ $|0.70|)$.

\begin{tabular}{|l|r|r|r|r|r|r|r|r|r|r|}
\hline Predictor & \multicolumn{1}{|c|}{ Dirt } & \multicolumn{1}{l|}{ Forbs } & \multicolumn{1}{l}{ Grams } & \multicolumn{1}{l}{ Litter } & \multicolumn{1}{l}{ Rock } & \multicolumn{1}{l}{ Shrubs } & \multicolumn{1}{l}{ Trees } & Woodrat & Elevation & Depth \\
\hline Dirt & 1 & -0.04 & 0.33 & -0.16 & -0.68 & 0.02 & -0.04 & 0.03 & -0.21 & -0.08 \\
\hline Forbs & -0.04 & 1 & -0.05 & -0.2 & 0.09 & -0.4 & -0.26 & -0.24 & 0.44 & 0 \\
\hline Grams & 0.33 & -0.05 & 1 & -0.35 & 0.05 & -0.31 & -0.39 & -0.51 & 0.13 & 0.18 \\
\hline Litter & -0.16 & -0.2 & -0.35 & 1 & -0.58 & 0.28 & 0.33 & 0.41 & -0.23 & 0.21 \\
\hline Rock & -0.68 & 0.09 & 0.05 & -0.58 & 1 & -0.18 & -0.23 & -0.31 & 0.35 & -0.09 \\
\hline Shrubs & 0.02 & -0.4 & -0.31 & 0.28 & -0.18 & 1 & -0.21 & 0.45 & -0.51 & -0.09 \\
\hline Trees & -0.04 & -0.26 & -0.39 & 0.33 & -0.23 & -0.21 & 1 & 0.23 & -0.14 & 0.13 \\
\hline Woodrat & 0.03 & -0.24 & -0.51 & 0.41 & -0.31 & 0.45 & 0.23 & 1 & -0.65 & 0.09 \\
\hline Elevation & -0.21 & 0.44 & 0.13 & -0.23 & 0.35 & -0.51 & -0.14 & -0.65 & 1 & -0.2 \\
\hline Depth & -0.08 & 0 & 0.18 & 0.21 & -0.09 & -0.09 & 0.13 & 0.09 & -0.2 & 1 \\
\hline
\end{tabular}

Table 4. Correlation matrix of microclimate variables, using Pearson product-moment correlation coefficient $(r)$. Values of $r \geq 10.70 \mid$ are in bold, and these are all significant $(P$ $\leq 0.0002)$.

\begin{tabular}{|c|c|c|c|c|c|c|c|c|c|c|c|c|}
\hline Predictor & Elevation & Summer & Hottest 10 & Mean & Mode & Min & Max & Days $<0$ & Days $<-5$ & Coldest 10 & Days $>20$ & $\mathrm{CV}$ \\
\hline Elevation & 1 & -0.73 & -0.59 & -0.7 & 0.04 & 0.24 & -0.38 & 0.15 & -0.04 & 0.21 & -0.4 & 0.61 \\
\hline Summer & -0.73 & 1 & 0.95 & 0.97 & 0.04 & -0.26 & 0.81 & -0.2 & 0.03 & -0.17 & 0.82 & -0.64 \\
\hline Hottest 10 & -0.59 & 0.95 & 1 & 0.94 & 0 & -0.23 & 0.89 & -0.14 & 0 & -0.12 & 0.87 & -0.55 \\
\hline Mean & -0.7 & 0.97 & 0.94 & 1 & 0.16 & -0.16 & 0.78 & -0.31 & -0.07 & -0.04 & 0.85 & -0.71 \\
\hline Mode & 0.04 & 0.04 & 0 & 0.16 & 1 & 0.48 & -0.07 & -0.59 & -0.4 & 0.53 & 0 & -0.55 \\
\hline Min & 0.24 & -0.26 & -0.23 & -0.16 & 0.48 & 1 & -0.11 & -0.58 & -0.87 & 0.91 & -0.31 & -0.29 \\
\hline Max & -0.38 & 0.81 & 0.89 & 0.78 & -0.07 & -0.11 & 1 & -0.16 & -0.03 & -0.01 & 0.77 & -0.41 \\
\hline Days $<0$ & 0.15 & -0.2 & -0.14 & -0.31 & -0.59 & -0.58 & -0.16 & 1 & 0.61 & -0.7 & -0.14 & 0.69 \\
\hline Days $<-5$ & -0.04 & 0.03 & 0 & -0.07 & -0.4 & -0.87 & -0.03 & 0.61 & 1 & -0.86 & 0.12 & 0.42 \\
\hline Coldest 10 & 0.21 & -0.17 & -0.12 & -0.04 & 0.53 & 0.91 & -0.01 & -0.7 & -0.86 & 1 & -0.15 & -0.37 \\
\hline Days $>20$ & -0.4 & 0.82 & 0.87 & 0.85 & 0 & -0.31 & 0.77 & -0.14 & 0.12 & -0.15 & 1 & -0.41 \\
\hline $\mathrm{CV}$ & 0.61 & -0.64 & -0.55 & -0.71 & -0.55 & -0.29 & -0.41 & 0.69 & 0.42 & -0.37 & -0.41 & 1 \\
\hline
\end{tabular}


Table 5. Sites surveyed in 2009 and 2010. Some sites were only surveyed in 2009, so there are no occupancy results listed in 2010 (NA). "Final Occupancy" denotes the final status used in analyses.

\begin{tabular}{|c|c|c|c|c|c|c|c|}
\hline Site & Region & $\begin{array}{l}\text { Elevation } \\
\text { (m) }\end{array}$ & Historic & $\begin{array}{l}\text { Data- } \\
\text { logger }\end{array}$ & $\begin{array}{l}\text { Occupancy } \\
2009\end{array}$ & $\begin{array}{l}\text { Occupancy } \\
2010\end{array}$ & $\begin{array}{l}\text { Final } \\
\text { Occupancy }\end{array}$ \\
\hline $\begin{array}{l}1 \text { mi N Glassburner } \\
\text { Meadows }\end{array}$ & Mineral & 2134 & $\mathrm{Y}$ & 1 & Abandoned & Abandoned & Abandoned \\
\hline Bluff Falls & Mineral & 2012 & $\mathrm{Y}$ & 1 & Abandoned & Abandoned & Abandoned \\
\hline $\begin{array}{l}\text { Morgan Mountain, W } \\
\text { slope }\end{array}$ & Mineral & 1905 & $\mathrm{~N}$ & 1 & Abandoned & Abandoned & Abandoned \\
\hline $\begin{array}{l}\text { Morgan Mountain, } \\
\text { crest }\end{array}$ & Mineral & 2073 & $\mathrm{~N}$ & 1 & Abandoned & Abandoned & Abandoned \\
\hline $\begin{array}{l}\text { Morgan Mountain, } \\
\text { NE slope }\end{array}$ & Mineral & 2012 & $\mathrm{~N}$ & 1 & Abandoned & Abandoned & Abandoned \\
\hline $\begin{array}{l}\text { Turner Mountain } \\
\text { Lookout }\end{array}$ & Mineral & 2042 & $\mathrm{~N}$ & 2 & Abandoned & Abandoned & Abandoned \\
\hline $1 \mathrm{mi} \mathrm{S}$ Bluff Falls & Mineral & 1966 & $\mathrm{~N}$ & 1 & Abandoned & Abandoned & Abandoned \\
\hline $\begin{array}{l}\text { Morgan Mountain, E } \\
\text { slope } 1\end{array}$ & Mineral & 1951 & $\mathrm{~N}$ & 0 & Abandoned & NA & Abandoned \\
\hline $\begin{array}{l}\text { Morgan Mountain, S } \\
\text { slope }\end{array}$ & Mineral & 2042 & $\mathrm{~N}$ & 0 & Abandoned & NA & Abandoned \\
\hline $\begin{array}{l}\text { Morgan Mountain, E } \\
\text { slope } 2\end{array}$ & Mineral & 1905 & $\mathrm{~N}$ & 0 & No sign & NA & Unknown \\
\hline $\begin{array}{l}\text { Head of Kings Creek } \\
\text { Canyon }\end{array}$ & LVNP & 2423 & $\mathrm{Y}$ & 1 & Occupied & Occupied & Occupied \\
\hline Upper Kings Creek & LVNP & 2530 & $\mathrm{~N}$ & 1 & Occupied & Occupied & Occupied \\
\hline Emerald Lake & LVNP & 2484 & $\mathrm{Y}$ & 2 & Abandoned & Occupied & Occupied \\
\hline Kings Creek Falls & LVNP & 2164 & $\mathrm{Y}$ & 2 & Occupied & Occupied & Occupied \\
\hline Reading Peak & LVNP & 2438 & $\mathrm{Y}$ & 1 & Occupied & Occupied & Occupied \\
\hline $\begin{array}{l}\text { Shadow Lake, NNW } \\
\text { shore }\end{array}$ & LVNP & 2316 & $\mathrm{~N}$ & 0 & No sign & NA & Unknown \\
\hline Mosquito Pond & LVNP & 2256 & $\mathrm{~N}$ & 1 & Occupied & Occupied & Occupied \\
\hline Paradise Meadow & LVNP & 2271 & $\mathrm{~N}$ & 2 & Occupied & Occupied & Occupied \\
\hline $\begin{array}{l}\text { Lower Kings Creek } \\
\text { Meadow }\end{array}$ & LVNP & 2195 & $\mathrm{Y}$ & 0 & Abandoned & NA & Abandoned \\
\hline $\begin{array}{l}\text { Flatiron Ridge, NE } \\
\text { slope } 1\end{array}$ & LVNP & 1661 & $\mathrm{Y}$ & 1 & Occupied & Occupied & Occupied \\
\hline $\begin{array}{l}\text { Flatiron Ridge, NE } \\
\text { slope } 2\end{array}$ & LVNP & 1676 & $\mathrm{~N}$ & 2 & Occupied & Occupied & Occupied \\
\hline Butte Lake, N shore & LVNP & 1844 & $\mathrm{Y}$ & 2 & Occupied & Occupied & Occupied \\
\hline Fantastic Lava Beds & LVNP & 1844 & $\mathrm{Y}$ & 1 & Occupied & Occupied & Occupied \\
\hline $\begin{array}{l}\text { Prospect Peak, SE } \\
\text { face }\end{array}$ & LVNP & 2164 & $\mathrm{Y}$ & 2 & Occupied & Occupied & Occupied \\
\hline $\begin{array}{l}\text { Lassen Peak } \\
\text { Trailhead }\end{array}$ & LVNP & 2637 & $\mathrm{~N}$ & 1 & Occupied & Abandoned & Occupied \\
\hline Ski Heil & LVNP & 2637 & $\mathrm{~N}$ & 1 & Occupied & Occupied & Occupied \\
\hline Eagle Peak, crest & LVNP & 2789 & $\mathrm{~N}$ & 1 & Occupied & Abandoned & Occupied \\
\hline Lassen Peak trail & LVNP & 2789 & $\mathrm{Y}$ & 0 & Occupied & NA & Occupied \\
\hline $\begin{array}{l}\text { Kings Creek Falls } \\
\text { Trailhead }\end{array}$ & LVNP & 2225 & $\mathrm{~N}$ & 1 & Abandoned & Abandoned & Abandoned \\
\hline $\begin{array}{l}\text { Eagle Lake Field } \\
\text { Station }\end{array}$ & Eagle Lake & 1615 & $\mathrm{Y}$ & 1 & Abandoned & Abandoned & Abandoned \\
\hline
\end{tabular}




\begin{tabular}{|l|l|r|l|r|l|l|l|} 
Papoose Meadow & Eagle Lake & 1646 & $\mathrm{Y}$ & 2 & Occupied & Occupied & Occupied \\
\hline $\begin{array}{l}\text { Mahogany Peak, W } \\
\text { base }\end{array}$ & Eagle Lake & 1676 & $\mathrm{~N}$ & 1 & Occupied & Occupied & Occupied \\
\hline $\begin{array}{l}\text { Gallatin Peak, W } \\
\text { base }\end{array}$ & Eagle Lake & 1631 & $\mathrm{Y}$ & 0 & Occupied & Occupied & Occupied \\
\hline $\begin{array}{l}\text { Black Mountain, SW } \\
\text { crest }\end{array}$ & Eagle Lake & 1859 & $\mathrm{~N}$ & 1 & Abandoned & Abandoned & Abandoned \\
\hline 3 mi NE Horse Lake & $\begin{array}{l}\text { Madeline } \\
\text { Plains }\end{array}$ & 1996 & $\mathrm{Y}$ & 1 & No sign & No sign & Abandoned \\
\hline $\begin{array}{l}\text { McDonald Peak, SE } \\
\text { slope }\end{array}$ & $\begin{array}{l}\text { Madeline } \\
\text { Plains }\end{array}$ & 1768 & $\mathrm{Y}$ & 1 & Abandoned & Abandoned & Abandoned \\
\hline
\end{tabular}

Table 6. Number of historic and additional (opportunistic) sites surveyed for pikas, including those with dataloggers and those without, showing pika occupation status. The 29 datalogger sites are included in the total sites surveyed.

\begin{tabular}{|l|r|r|r|}
\hline & Occupied & Abandoned & \multicolumn{1}{|c|}{ Total } \\
\hline Sites Surveyed & 11 & 6 & 37 \\
\hline Historic & 6 & 11 & 17 \\
\hline Additional & 9 & & 29 \\
\hline Datalogger Sites & 8 & 5 & 14 \\
\hline Historic & 7 & 7 & 15 \\
\hline Additional
\end{tabular}

Table 7. Detection of pika sign in surveys. "First sign" is the first sign discovered during surveys. "All surveys" pools the types of sign found in all occupancy-known surveys (both occupied and abandoned). The last two columns (all surveys and occupied surveys) refer to the percentage of surveys that particular sign was detected throughout each complete survey. The sample sizes (n) are not all the same because first sign was not recorded in every survey conducted, and the detection of all types of sign was not recorded for all surveys.

\begin{tabular}{|l|r|r|r|r|}
\hline Sign Type & $\begin{array}{c}\text { First Sign All } \\
\text { Surveys (\%) } \\
(\mathrm{n}=71)\end{array}$ & $\begin{array}{c}\text { First Sign Occupied } \\
\text { Surveys (\%) } \\
(\mathrm{n}=40)\end{array}$ & $\begin{array}{c}\text { In All Surveys } \\
(\%) \\
(\mathrm{n}=77)\end{array}$ & $\begin{array}{c}\text { In Occupied } \\
\text { Surveys (\%) } \\
(\mathrm{n}=38)\end{array}$ \\
\hline Pellets & 94.4 & 90 & 92.2 & 100 \\
\hline Haypiles & 1.4 & 2.5 & 57.1 & 60.5 \\
\hline Calls & 2.8 & 5 & 28.6 & 57.9 \\
\hline Sightings & 1.4 & 2.5 & 7.8 & 14.8 \\
\hline
\end{tabular}


Table 8. Average habitat variables in occupied $(n=17)$ and abandoned sites $(n=12)$. Relative covers of vegetation, proportions of substrates, and talus depth $(\mathrm{cm})$ are averaged for each site, and standard errors (SE) are given. The test statistic and $P$ values for each Welch's two-sample $t$-test, comparing occupied to abandoned sites, is shown. "Woodrat" gives the total number of sites with woodrat sign, not an average.

\begin{tabular}{|c|c|c|c|c|c|c|}
\hline \multirow[t]{2}{*}{$\begin{array}{c}\text { Habitat } \\
\text { Variable } \\
\end{array}$} & \multicolumn{2}{|c|}{ Abandoned $(\mathrm{n}=12)$} & \multicolumn{2}{|c|}{ Occupied $(n=17)$} & \multicolumn{2}{|c|}{$t$-test } \\
\hline & Mean & SE & Mean & SE & $t$-stat & $P$ value \\
\hline Shrubs* & 0.53 & 0.08 & 0.24 & 0.05 & 3.08 & 0.01 \\
\hline Litter* & 0.13 & 0.04 & 0.03 & 0.01 & 2.69 & 0.02 \\
\hline Graminoids* & 0.08 & 0.03 & 0.26 & 0.08 & -2.15 & 0.04 \\
\hline Forbs* & 0.08 & 0.05 & 0.26 & 0.07 & -2.06 & 0.05 \\
\hline Elevation(m) & 1968 & 47.48 & 2178 & 94.92 & -1.98 & 0.06 \\
\hline Rock* & 0.78 & 0.04 & 0.85 & 0.03 & -1.45 & 0.17 \\
\hline Depth $(\mathrm{cm})$ & 86.4 & 9.62 & 98.6 & 6.34 & -1.3 & 0.21 \\
\hline Trees $^{+}$ & 0.22 & 0.07 & 0.2 & 0.08 & 0.3 & 0.77 \\
\hline Dirt* & 0.09 & 0.04 & 0.09 & 0.02 & -0.18 & 0.86 \\
\hline Woodrat & 12 & NA & 8 & NA & 4.24 & 0.00 \\
\hline
\end{tabular}

* Variables were log- transformed before analysis

${ }^{+}$Variables were transformed by square root before analysis 
Table 9. Averages of microclimate variables in occupied and abandoned sites (temperatures are in Celsius) with standard error (SE). Temperatures from sites with multiple dataloggers were averaged. The test statistic and $P$ values for each Welch's two-sample $t$-test, comparing occupied to abandoned sites, is shown. The number of days below $-5^{\circ} \mathrm{C}$ and above $20^{\circ} \mathrm{C}$ were log-transformed before $t$-tests.

\begin{tabular}{|l|r|r|r|r|r|r|}
\hline Climate Variable & \multicolumn{2}{|c|}{$\begin{array}{c}\text { Abandoned } \\
(\mathbf{n}=12)\end{array}$} & \multicolumn{2}{c|}{$\begin{array}{c}\text { Occupied } \\
(\mathbf{n}=\mathbf{1 7})\end{array}$} & \multicolumn{2}{c|}{$t$-test } \\
\hline & Mean & \multicolumn{1}{c|}{ SE } & \multicolumn{1}{c|}{ Mean } & \multicolumn{1}{c|}{ SE } & \multicolumn{1}{c|}{-stat } & $\boldsymbol{P}$ value \\
\hline Summer & 14.19 & 1.13 & 10.18 & 1.04 & 2.61 & 0.02 \\
\hline Mean & 6.24 & 0.61 & 4.18 & 0.53 & 2.55 & 0.02 \\
\hline CV & 1.13 & 0.05 & 1.56 & 0.17 & -2.36 & 0.03 \\
\hline Hottest 10 days & 18 & 1.22 & 14.64 & 0.85 & 2.26 & 0.03 \\
\hline Days $<0^{\circ} \mathrm{C}$ & 58 & 9.83 & 98 & 16.50 & -2.08 & 0.05 \\
\hline Days $>20^{\circ} \mathrm{C}^{*}$ & 41.38 & 13.87 & 10.82 & 5.22 & 1.96 & 0.07 \\
\hline Maximum & 21.52 & 1.44 & 18.51 & 1.37 & 1.51 & 0.14 \\
\hline Mode & 0.64 & 0.16 & 0.30 & 0.22 & 1.25 & 0.22 \\
\hline Minimum & -7.85 & 1.01 & -6.58 & 0.95 & -0.91 & 0.37 \\
\hline Days $<-5^{\circ} \mathrm{C}^{*}$ & 5.50 & 1.70 & 4.82 & 1.23 & 0.28 & 0.78 \\
\hline Coldest 10 days & -3.44 & 0.69 & -3.38 & 0.58 & -0.07 & 0.95 \\
\hline
\end{tabular}

* Variables were log-transformed before analysis

Table 10. Results of univariate logistic regressions for habitat variables, predicting pika occupancy.

\begin{tabular}{|c|c|c|c|}
\hline Predictor & $\begin{array}{c}\text { Effect } \\
\text { sign }\end{array}$ & $-2 \log (L)$ & $P$ value \\
\hline Shrubs* & - & 30.682 & 0.013 \\
\hline Litter* & - & 29.599 & 0.028 \\
\hline Forbs* & + & 35.297 & 0.087 \\
\hline Graminoids* & + & 35.514 & 0.095 \\
\hline Elevation & + & 36.204 & 0.100 \\
\hline Rock* & + & 36.973 & 0.145 \\
\hline Depth & + & 37.428 & 0.184 \\
\hline Trees $^{+}$ & - & 39.246 & 0.764 \\
\hline Dirt* & + & 39.297 & 0.844 \\
\hline Woodrat & - & 26.920 & 0.993 \\
\hline
\end{tabular}

* Variables were log-transformed before analysis

${ }^{+}$Variables were transformed by square root before analysis 
Table 11. Candidate habitat models used to predict pika occupancy in additive logistic regressions. $\mathrm{K}$ is the number of fitted parameters, and $\Delta \mathrm{AIC}_{\mathrm{c}}$ is the difference between the indicated model and the best-supported model.

\begin{tabular}{|c|c|c|c|c|c|c|}
\hline Categories & Predictors (sign of effect) & $\mathbf{K}$ & $-2 \log L$ & $\mathrm{AIC}_{\mathrm{C}}$ & $\Delta \mathbf{A I C}_{\mathbf{C}}$ & $\begin{array}{l}\text { Evidence } \\
\text { ratio }^{-1}\end{array}$ \\
\hline $\begin{array}{l}\text { Vegetation + } \\
\text { Substrate }\end{array}$ & $\begin{array}{l}\text { Forbs*(+), Graminoids*(+), } \\
\text { Shrubs*(-), Trees }{ }^{+}(+), \operatorname{Dirt}^{*}(-), \\
\operatorname{Rock}^{*}(-), \operatorname{Litter}^{*}(-)\end{array}$ & 8 & 13.766 & 36.966 & 0 & 1.000 \\
\hline Substrate & $\operatorname{Dirt}^{*}(-), \operatorname{Rock}^{*}(-)$, Litter*(-) & 4 & 27.363 & 37.030 & 0.064 & 0.969 \\
\hline $\begin{array}{l}\text { Substrate + } \\
\text { Elevation }\end{array}$ & $\begin{array}{l}\text { Dirt*(-), Rock*(-), Litter*(-), } \\
\text { Elevation }(+)\end{array}$ & 5 & 25.693 & 38.302 & 1.336 & 0.513 \\
\hline Vegetation & $\begin{array}{l}\text { Forbs*(+), Graminoids*(+), } \\
\text { Shrubs*(-), Trees }{ }^{+}(+)\end{array}$ & 5 & 27.346 & 39.955 & 2.989 & 0.224 \\
\hline Elevation & Elevation $(+)$ & 2 & 36.204 & 40.666 & 3.700 & 0.157 \\
\hline $\begin{array}{l}\text { Vegetation + } \\
\text { Substrate + } \\
\text { Elevation }\end{array}$ & $\begin{array}{l}\text { Forbs*(+), Graminoids*(+), } \\
\text { Shrubs*(-), Trees }{ }^{+}(+), \text {Dirt*(-), } \\
\left.\text { Rock*(-), Litter*(-), Elevation }^{*}+\right)\end{array}$ & 9 & 13.717 & 41.191 & 4.225 & 0.121 \\
\hline Null & Intercept only & 1 & 39.336 & 41.484 & 4.518 & 0.104 \\
\hline $\begin{array}{l}\text { Vegetation }+ \\
\text { Elevation }\end{array}$ & $\begin{array}{l}\text { Forbs*(+), Graminoids*(+), } \\
\text { Shrubs*(-), Trees }{ }^{+}(+), \text {Elevation } \\
(+)\end{array}$ & 6 & 27.343 & 43.161 & 6.195 & 0.045 \\
\hline
\end{tabular}

* Variables were log-transformed before analysis

${ }^{+}$Variables were transformed by square root before analysis

Table 12. Results of univariate logistic regressions for microclimate variables, predicting pika occupancy.

\begin{tabular}{|l|c|r|r|}
\hline \multicolumn{1}{|c|}{ Predictor } & $\begin{array}{c}\text { Effect } \\
\text { sign }\end{array}$ & $-\mathbf{- 2} \log (\mathbf{L})$ & $\boldsymbol{P}$ value \\
\hline Summer & - & 35.151 & 0.028 \\
\hline Mean & - & 35.379 & 0.028 \\
\hline Hottest 10 & - & 36.177 & 0.037 \\
\hline Days $>20^{*}$ & - & 37.425 & 0.058 \\
\hline CV & + & 35.191 & 0.068 \\
\hline Days $<0$ & + & 37.644 & 0.089 \\
\hline Max & - & 39.119 & 0.159 \\
\hline Mode & - & 40.008 & 0.269 \\
\hline Min & + & 40.501 & 0.368 \\
\hline Days <-5* & - & 41.253 & 0.774 \\
\hline Coldest 10 & + & 41.329 & 0.935 \\
\hline
\end{tabular}

* Variables were log-transformed before analysis 
Table 13. Candidate microclimate models used to predict pika occupancy in logistic regression. $\mathrm{K}$ is the number of fitted parameters, and $\Delta \mathrm{AIC}_{\mathrm{c}}$ is the difference between the indicated model and the best-supported model.

\begin{tabular}{|c|c|c|c|c|c|c|}
\hline Categories & Predictors (sign of effect) & $\mathbf{K}$ & $-2 \log L$ & $\mathbf{A I C}_{\mathbf{C}}$ & $\Delta \mathbf{A I C} \mathbf{C}_{\mathbf{C}}$ & $\begin{array}{c}\text { Evidence } \\
\text { ratio }^{-1}\end{array}$ \\
\hline Acute Cold & Min (+), Days $<0(+)$ & 3 & 26.996 & 33.956 & 0 & 1.000 \\
\hline $\begin{array}{l}\text { Acute Cold + Chronic } \\
\text { Heat }\end{array}$ & $\begin{array}{l}\text { Min }(+), \text { Days }<0(+), \text { Summer } \\
(-)\end{array}$ & 4 & 25.594 & 35.261 & 1.305 & 0.521 \\
\hline $\begin{array}{l}\text { Acute Cold }+ \text { Acute } \\
\text { Heat }\end{array}$ & $\begin{array}{l}\text { Min (+), Days }<0(+), \text { Days }> \\
20^{*}(-)\end{array}$ & 4 & 26.453 & 36.120 & 2.164 & 0.339 \\
\hline Chronic Heat & Summer (-) & 2 & 33.151 & 37.613 & 3.657 & 0.161 \\
\hline Acute Heat & Days $>20 *(-)$ & 2 & 35.425 & 39.887 & 5.931 & 0.052 \\
\hline $\begin{array}{l}\text { Chronic Heat + } \\
\text { Chronic Cold }\end{array}$ & $\begin{array}{l}\text { Summer (-), Coldest } 10(+) \text {, } \\
\text { Mode }(-)\end{array}$ & 4 & 31.363 & 41.030 & 7.074 & 0.029 \\
\hline Null & Intercept only & 1 & 39.336 & 41.484 & 7.528 & 0.023 \\
\hline Chronic Cold & Coldest $10(+)$, Mode (-) & 3 & 37.326 & 44.286 & 10.330 & 0.006 \\
\hline
\end{tabular}

* Variables were log-transformed before analysis 
Table 14. Final models used to predict pika occupancy in logistic regression, combining microclimate and habitat characteristics. $\mathrm{K}$ is the number of fitted parameters, and $\Delta \mathrm{AIC}_{\mathrm{c}}$ is the difference between the indicated model and the best-supported model.

\begin{tabular}{|c|c|c|c|c|c|c|}
\hline Categories & Predictors (sign of effect) & $\mathbf{K}$ & $-2 \log \mathrm{L}$ & $\mathrm{AIC}_{\mathrm{C}}$ & $\Delta \mathrm{AIC}_{\mathbf{C}}$ & $\begin{array}{c}\text { Evidence } \\
\text { ratio }^{-1}\end{array}$ \\
\hline Substrate + Acute Cold & $\begin{array}{l}\text { Dirt*(-), Rock*(-), Litter*(-), } \\
\operatorname{Min}(+), \text { Days }<0(+)\end{array}$ & 6 & 15.579 & 31.397 & 0 & 1.000 \\
\hline $\begin{array}{l}\text { Vegetation + Acute } \\
\text { Cold }\end{array}$ & $\begin{array}{l}\text { Forbs*(+), Graminoids*(+), } \\
\text { Shrubs*(-), Trees }{ }^{+}(+), \operatorname{Min}(+), \\
\text { Days }<0(+)\end{array}$ & 7 & 13.668 & 33.001 & 1.604 & 0.448 \\
\hline $\begin{array}{l}\text { Substrate + Elevation } \\
+ \text { Acute Cold }\end{array}$ & $\begin{array}{l}\text { Dirt*(-), Rock*(-), Litter*(-), } \\
\text { Elevation }(+), \text { Min }(+), \text { Days }<0 \\
(+)\end{array}$ & 7 & 15.263 & 34.596 & 3.199 & 0.202 \\
\hline $\begin{array}{l}\text { Substrate + Acute Cold } \\
+ \text { Chronic Heat }\end{array}$ & $\begin{array}{l}\text { Dirt*(-), Rock*(-), Litter*(-), } \\
\text { Min (+), Days < } 0(+), \text { Summer } \\
(+)\end{array}$ & 7 & 15.565 & 34.898 & 3.501 & 0.174 \\
\hline $\begin{array}{l}\text { Vegetation }+ \text { Acute } \\
\text { Cold }+ \text { Chronic Heat }\end{array}$ & $\begin{array}{l}\text { Forbs*(+), Graminoids*(+), } \\
\text { Shrubs*(-), Trees }{ }^{+}(+), \text {Min }(+), \\
\text { Days }<0(+), \text { Summer }(-)\end{array}$ & 8 & 12.935 & 36.135 & 4.738 & 0.094 \\
\hline $\begin{array}{l}\text { Substrate + Elevation } \\
+ \text { Acute Cold + Acute } \\
\text { Heat }\end{array}$ & $\begin{array}{l}\text { Dirt*(-), Rock*(-), Litter*(-), } \\
\text { Elevation }(+), \text { Min }(+), \text { Days }<0 \\
(+), \text { Days }>20^{*}(+)\end{array}$ & 8 & 15.198 & 38.398 & 7.001 & 0.030 \\
\hline $\begin{array}{l}\text { Vegetation }+ \text { Substrate } \\
+ \text { Chronic Heat }\end{array}$ & $\begin{array}{l}\text { Forbs*(+), Graminoids*(+), } \\
\text { Shrubs*(-), Trees }{ }^{+}(+), \operatorname{Dirt} *(-), \\
\operatorname{Rock}^{*}(-), \operatorname{Litter}^{*}(-), \operatorname{Summer}(+)\end{array}$ & 9 & 13.764 & 41.238 & 9.841 & 0.007 \\
\hline Null & intercept only & 1 & 39.336 & 41.484 & 10.087 & 0.006 \\
\hline $\begin{array}{l}\text { Vegetation + Chronic } \\
\text { Heat }\end{array}$ & $\begin{array}{l}\text { Forbs*(+), Graminoids*(+), } \\
\text { Shrubs*(-), Trees }{ }^{+}(+), \text {Summer } \\
(-)\end{array}$ & 6 & 25.969 & 41.787 & 10.390 & 0.006 \\
\hline
\end{tabular}

* Variables were log-transformed before analysis

${ }^{+}$Variables were transformed by square root before analysis 


\section{CHAPTER 2. FIGURES}

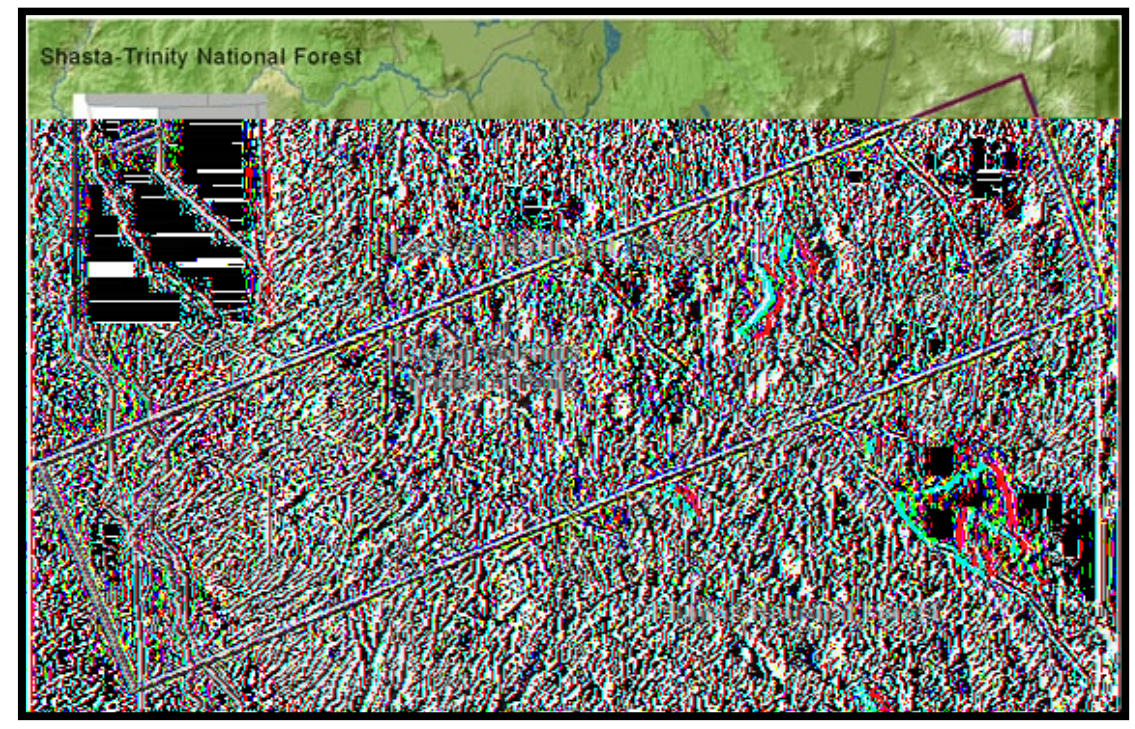

Figure 1. Lassen Transect. The rectangle marks the historic survey transect of Joseph Grinnell in the early 1900s. Pika sites are located primarily from just west of Lassen Volcanic National Park to east of Eagle Lake (large lake within transect). Map from Museum of Vertebrate Zoology, U.C. Berkeley. 


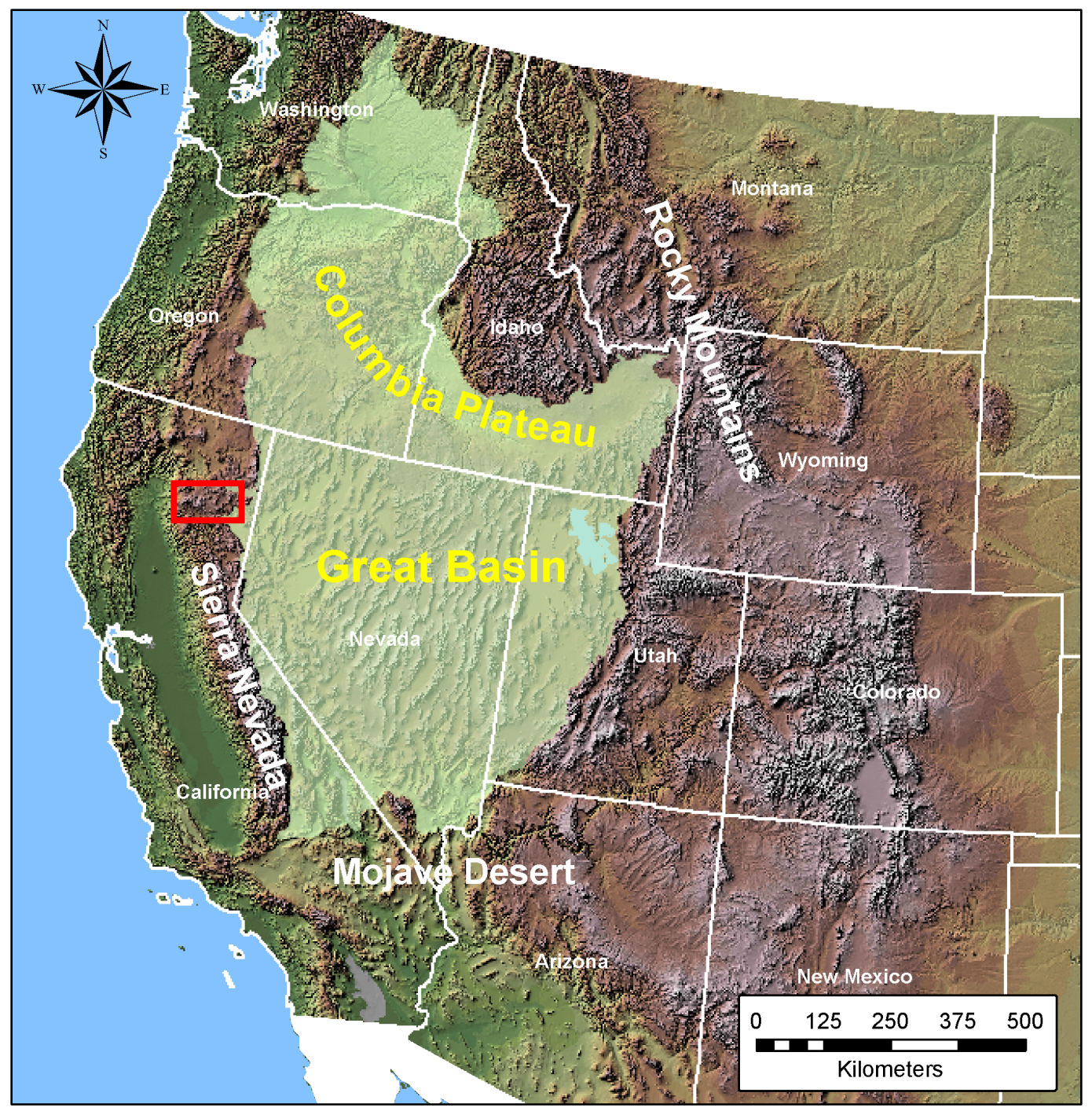

Figure 2. Map of Great Basin. Red square shows the Lassen Peak region study area, west of the Great Basin, and at the southern end of the Cascade Range (not labeled). Map has been adapted from NBII Great Basin. 


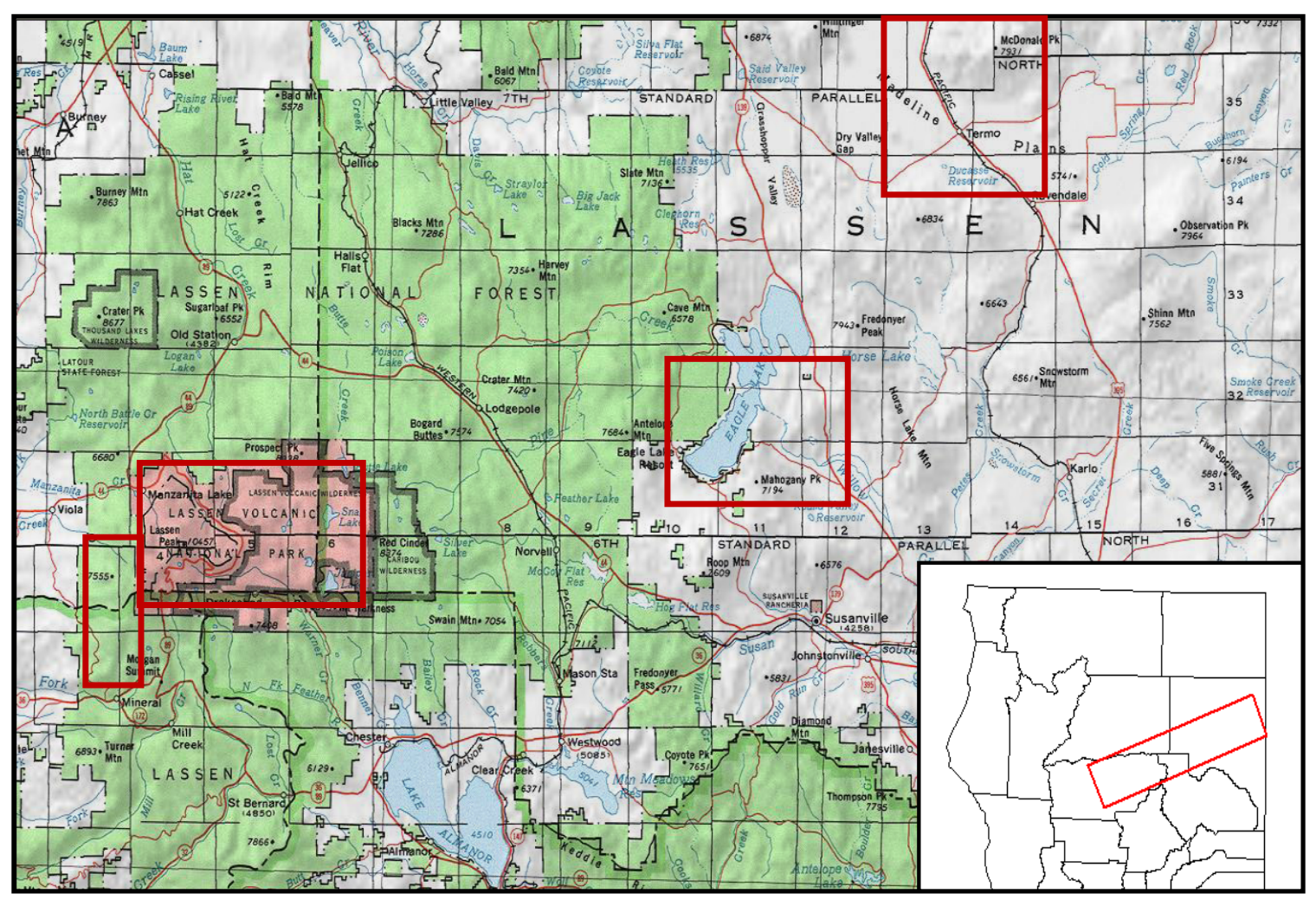

Figure 3. The four regions investigated in the Lassen Peak study area are outlined in red. From west to east: Mineral, Lassen Volcanic National Park (LVNP), Eagle Lake, and Madeline Plains. 


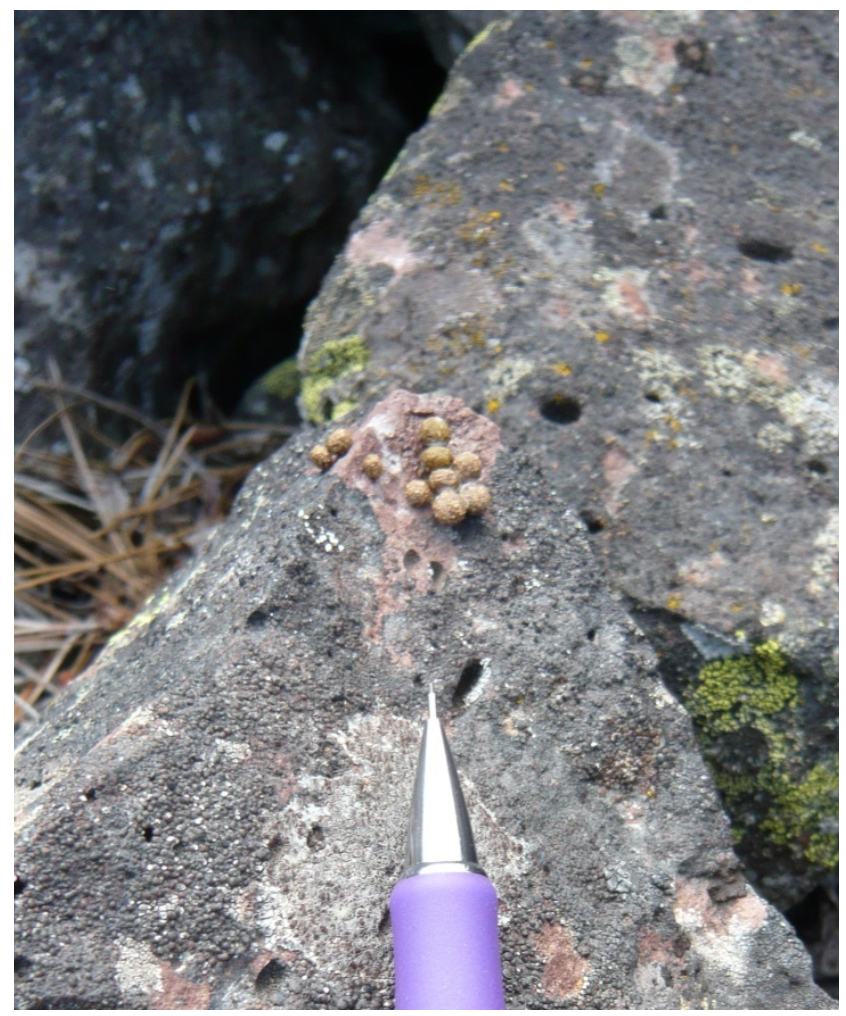

Figure 4. Perched pellets. These pellets are considered fresh, and were found in a location known to be occupied by pikas. Pikas on this slope in the Eagle Lake region were detected both directly (by sight and sound) and indirectly (through fresh pellets). Note the pellets are a light shade of brown and slightly green in color. Additionally, the pellets are "perched" almost vertically against the rock face. When crushed, these fresh pellets are fibrous inside, and green in color. 


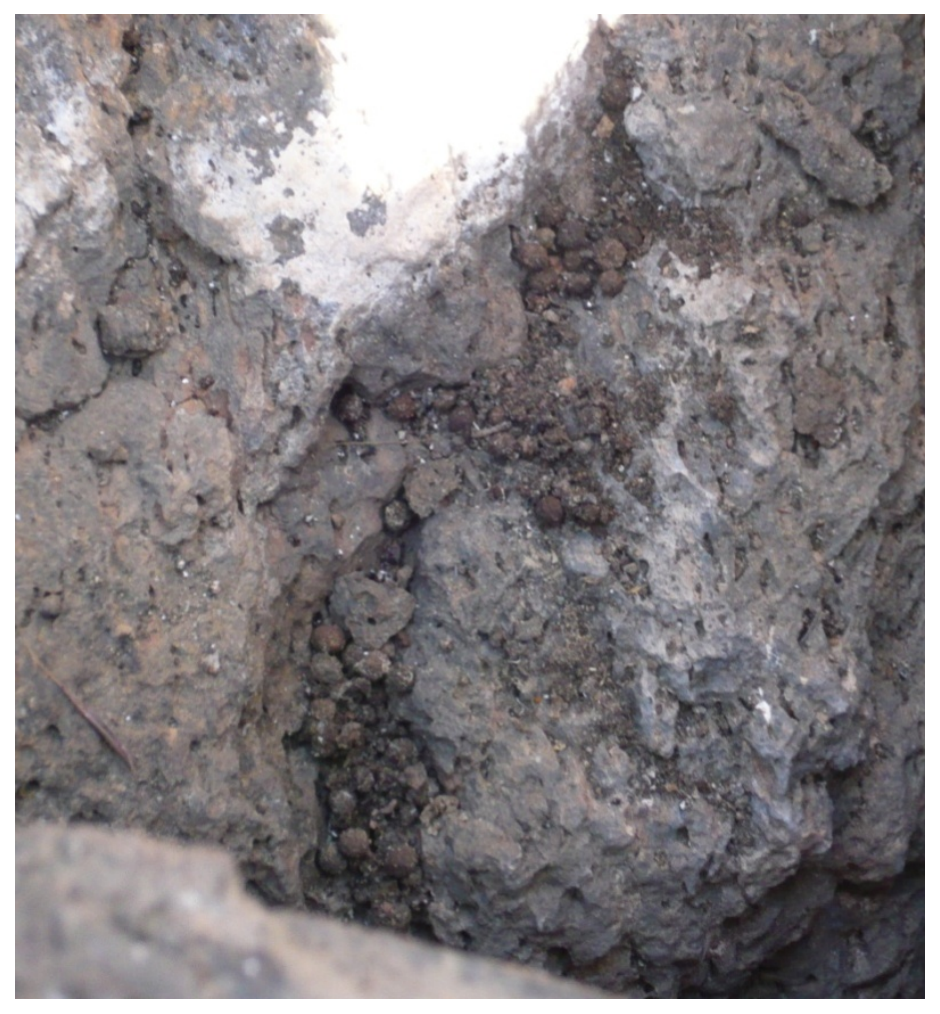

Figure 5. Settled pellets. The age of these pellets is unknown, but they are believed to be older than one season. They are brown to gray in color, and settled deep within the talus. Additionally, their consistency is not fibrous in texture; they turn to dust when crushed and are the same color inside as out.

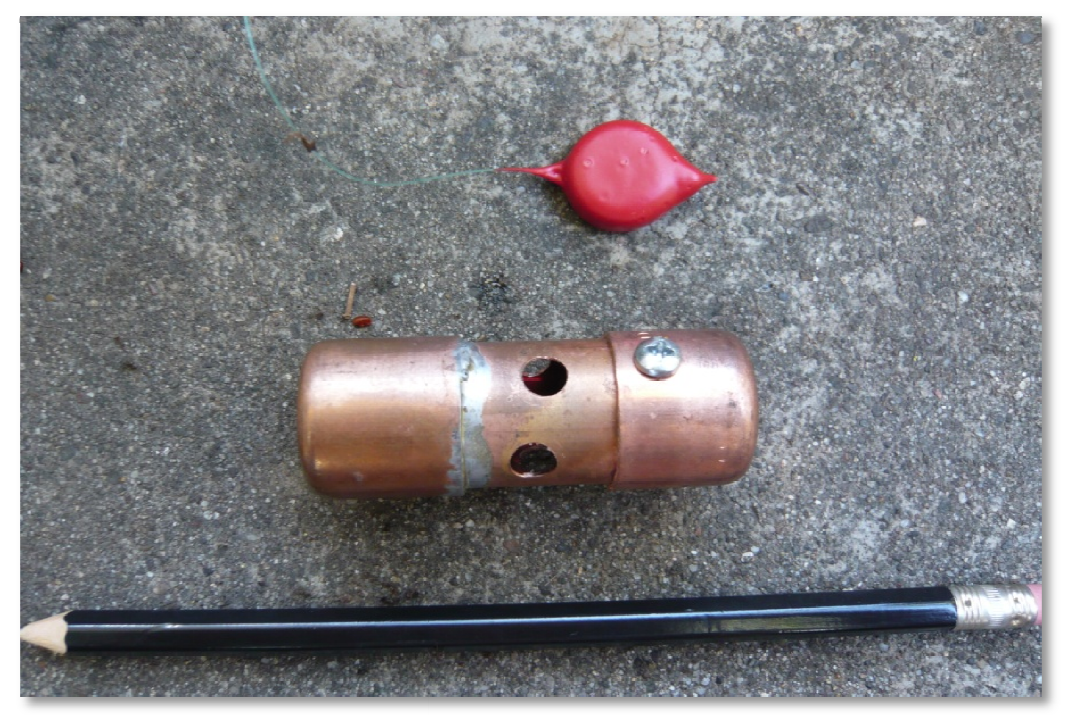

Figure 6. Copper capsule used to protect iButton thermal datalogger. The datalogger shown is coated in red Plasti Dip for waterproofing. 


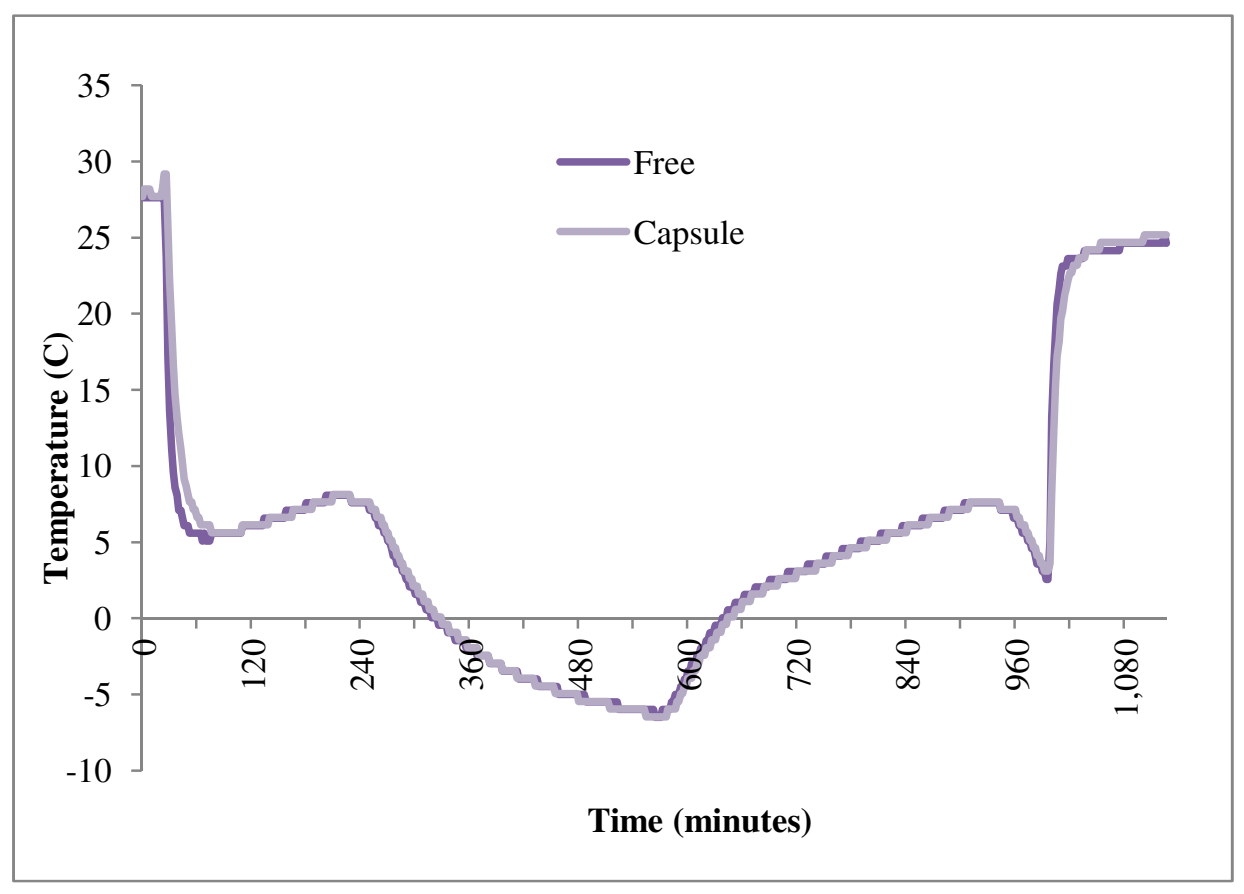

Figure 7. Datalogger trials between a datalogger in a copper capsule (Figure 6) and a datalogger with no capsule. The dataloggers were placed in an incubator, then a refrigerator, then a freezer, and finally placed back in the incubator.

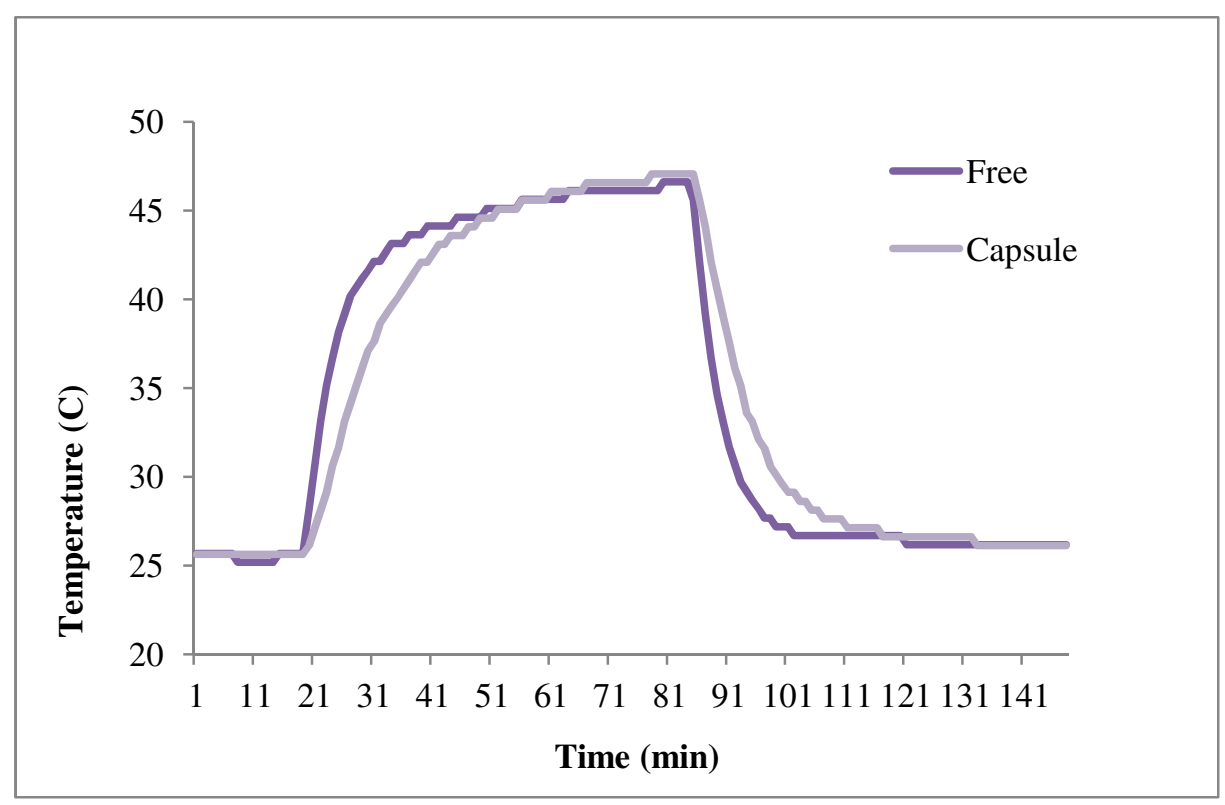

Figure 8. Datalogger trials between a datalogger in a copper capsule (Figure 6) and a datalogger with no capsule. The dataloggers were activated at room temperature, then moved to a $47^{\circ} \mathrm{C}$ incubator, and then returned to room temperature. 


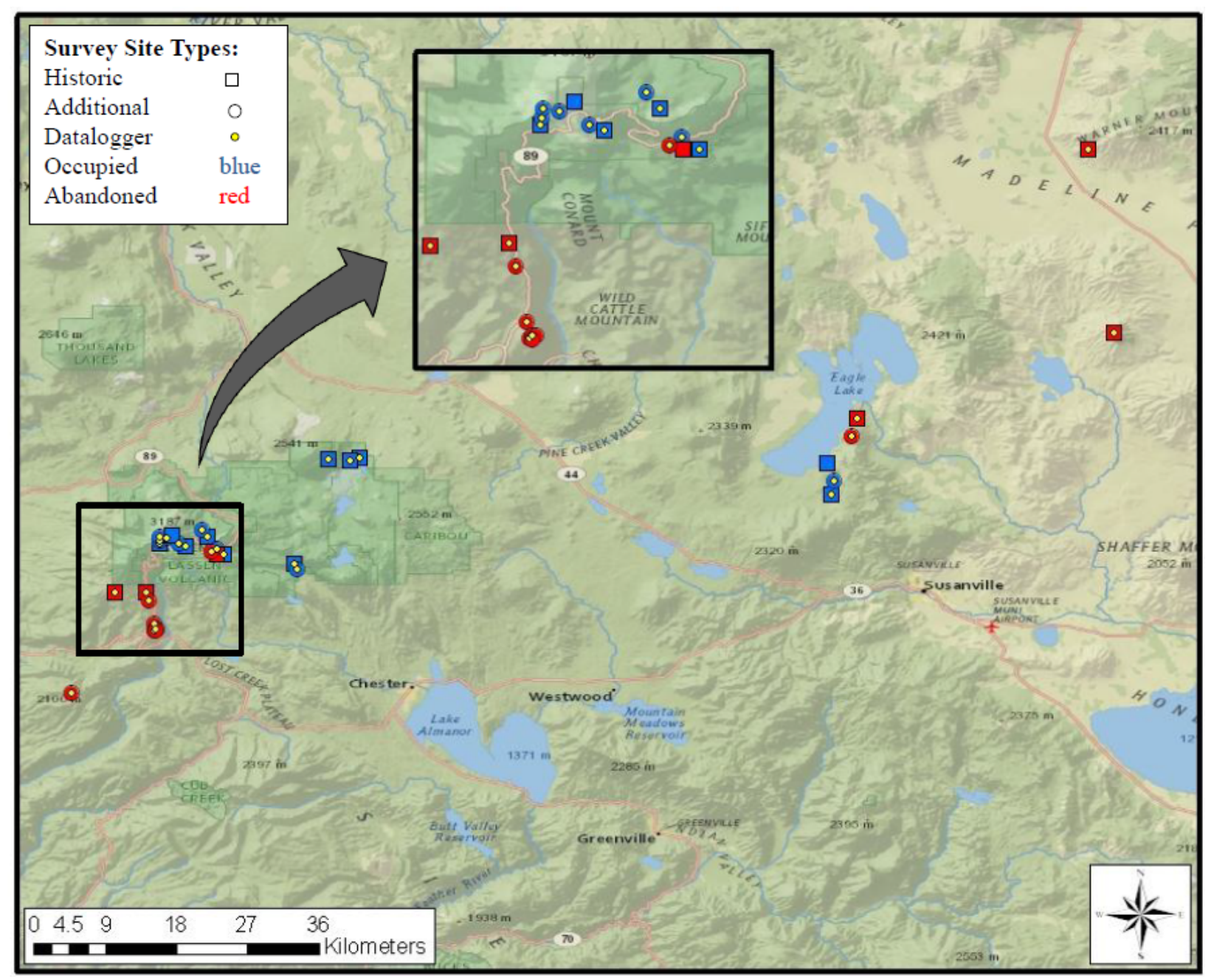

Figure 9. Survey sites in the Lassen Peak region, showing both occupied (blue) and abandoned (red) pika sites. Historic sites are denoted as squares; additional sites are circles, and the 29 sites with dataloggers are represented by yellow dots. Map inset shows part of the Mineral and LVNP regions at closer magnification. Squares and circles are not drawn to scale. 


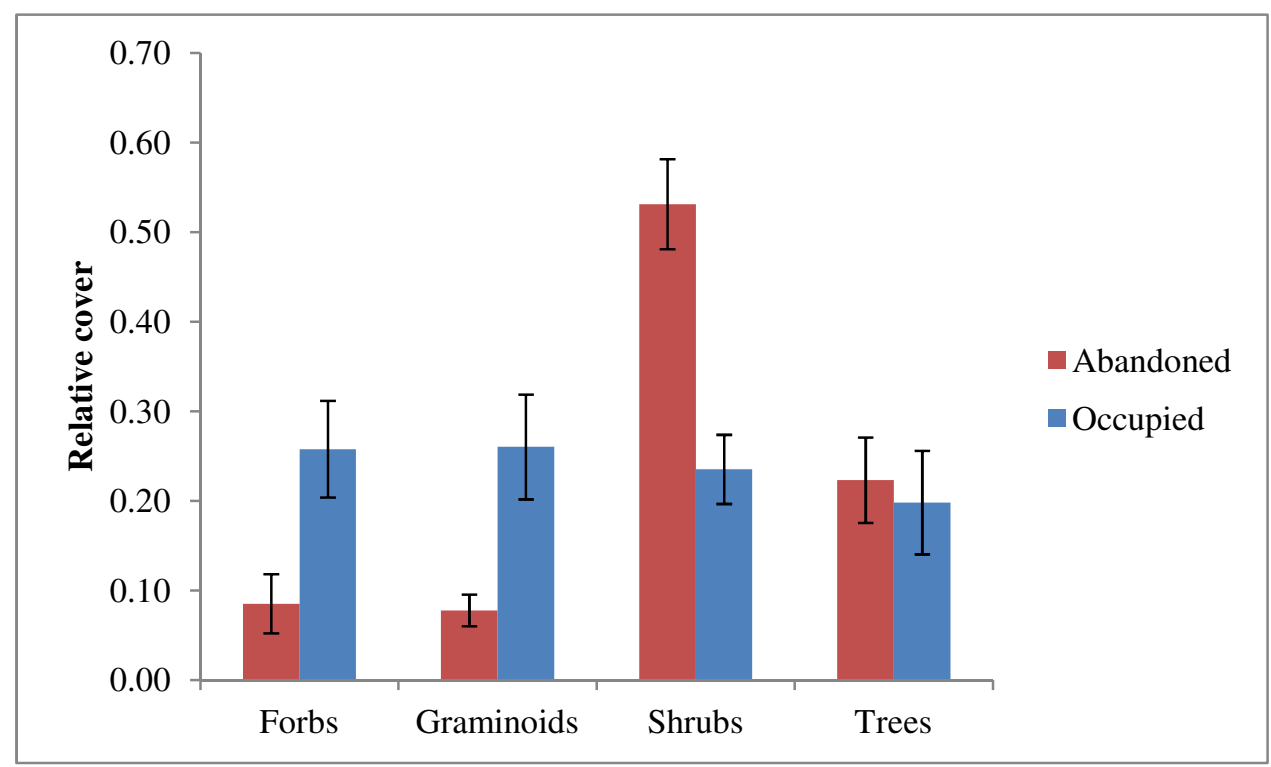

Figure 10. Average $( \pm \mathrm{SE})$ relative cover of vegetation classes in abandoned and occupied pika sites.

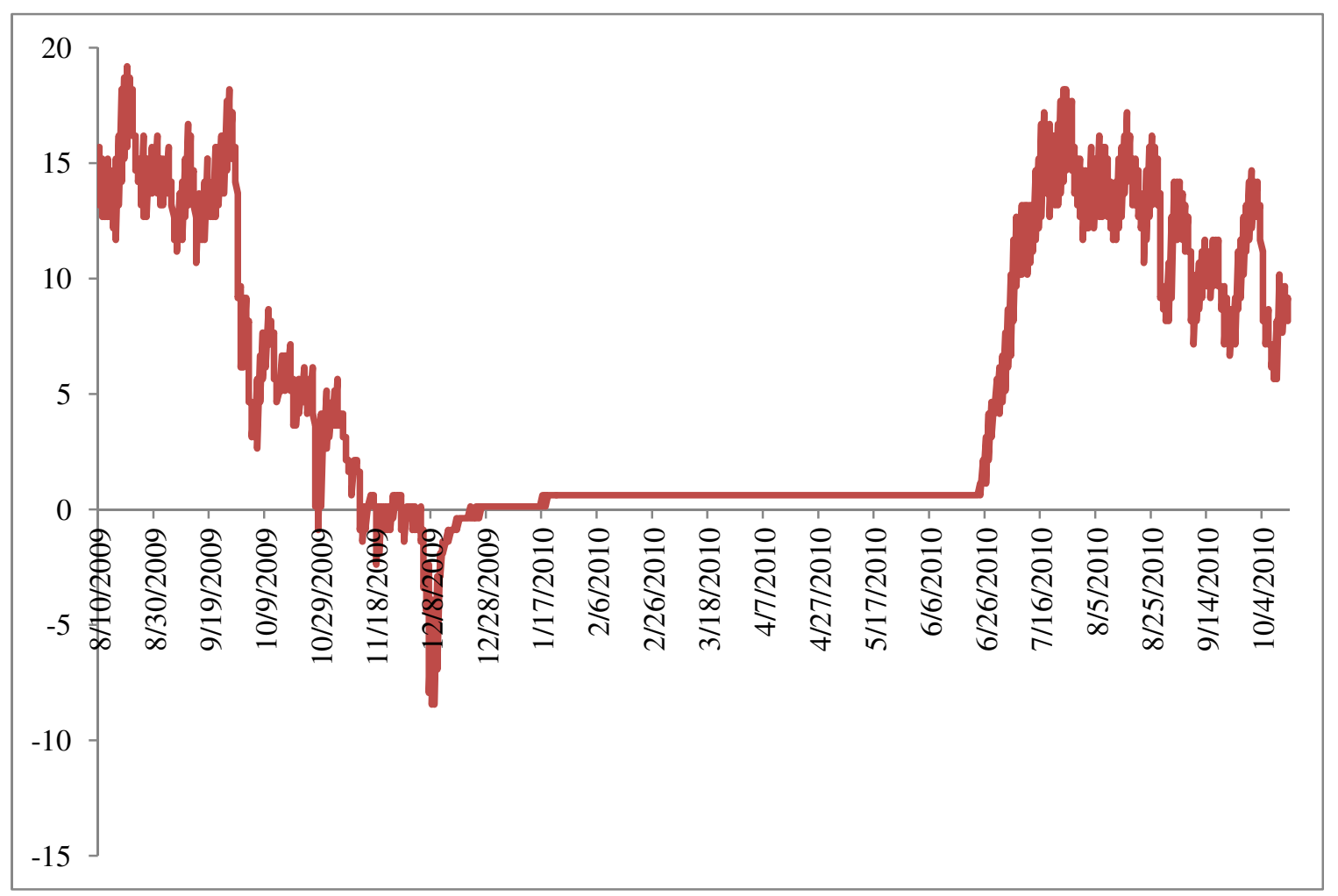

Figure 11. Yearly temperature profile of Bluff Falls, near Mineral, an abandoned site (elevation $2021 \mathrm{~m}$ ). 


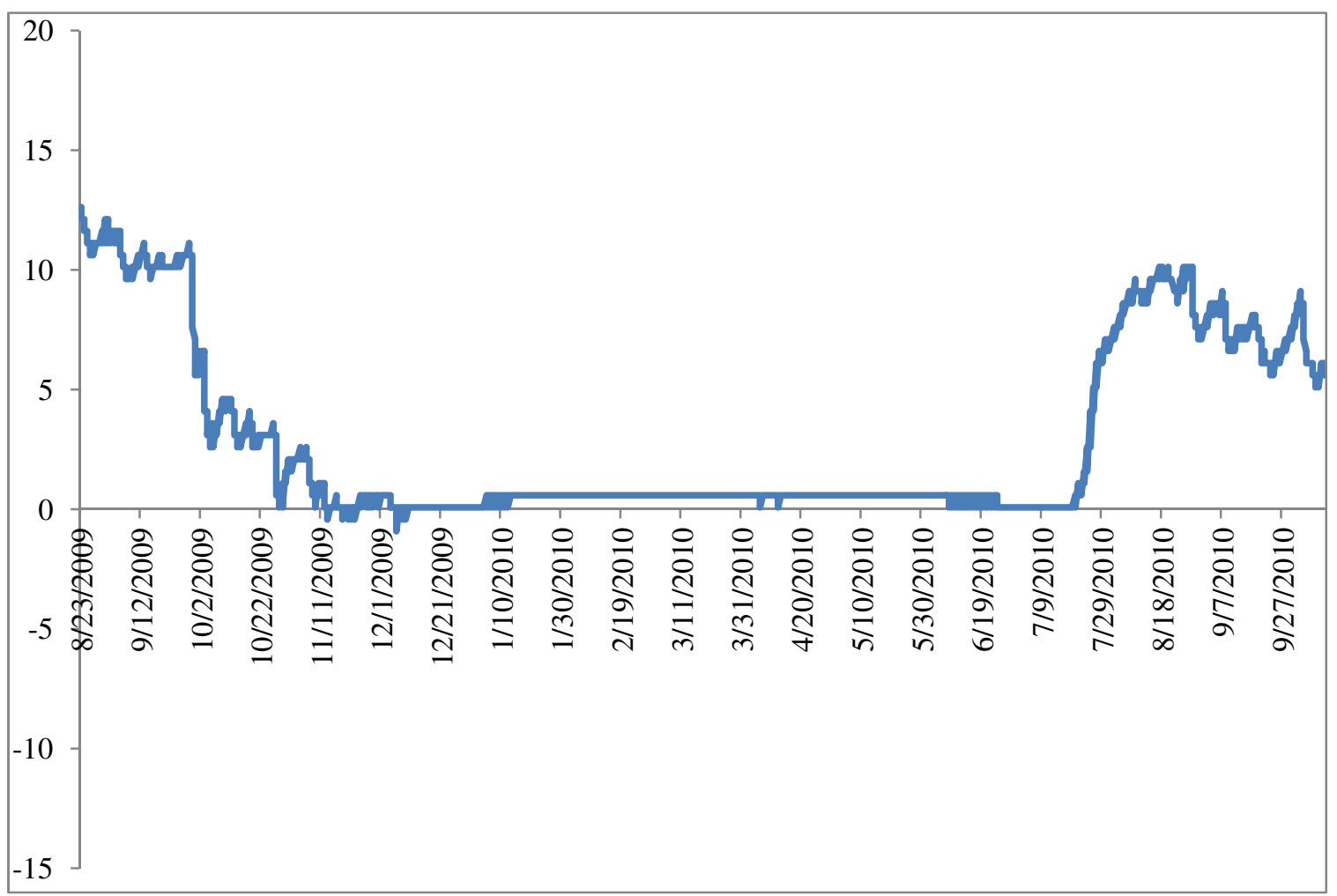

Figure 12. Yearly temperature profile of Paradise Meadow, LVNP, an occupied site (elevation $2253 \mathrm{~m}$ ). 


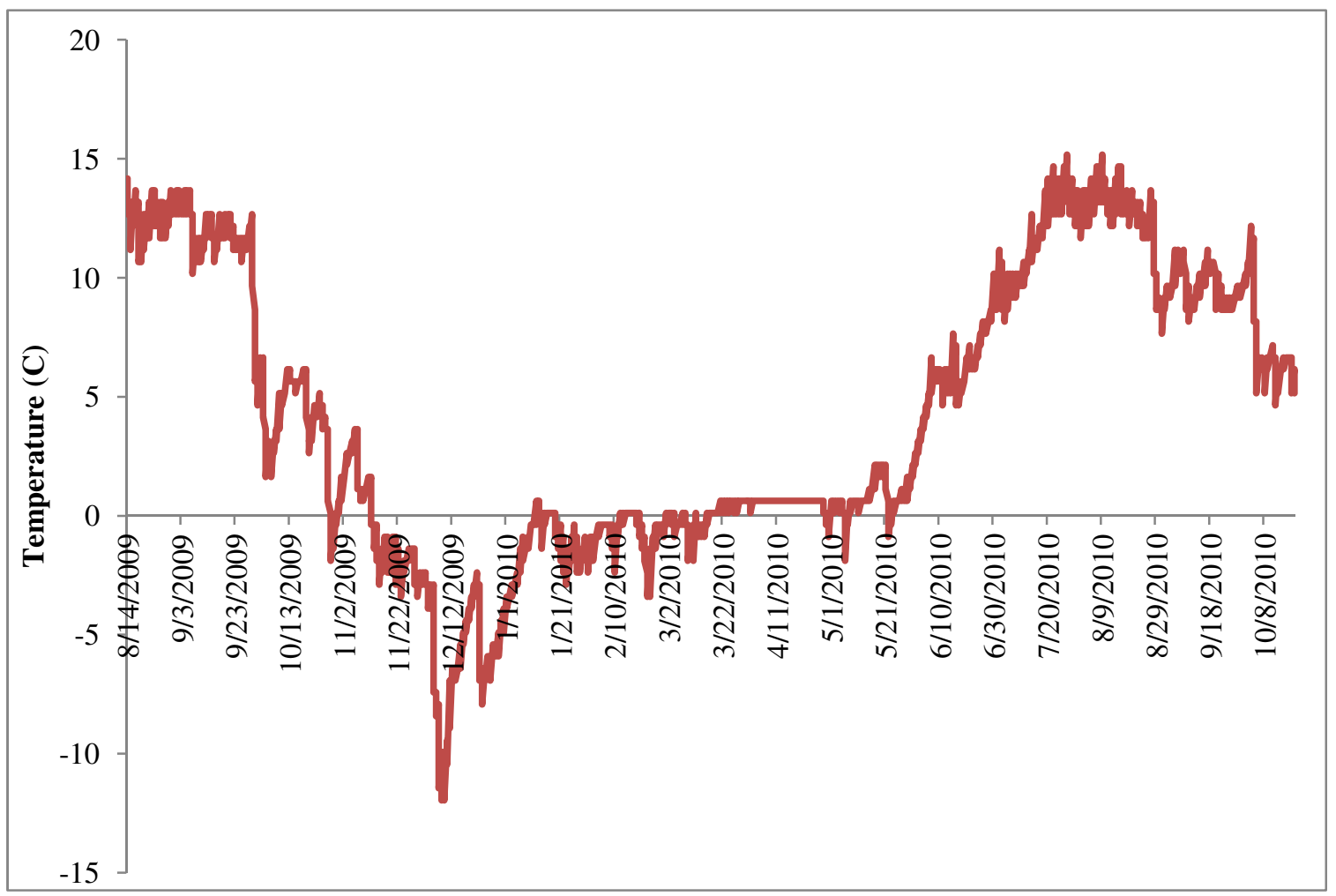

Figure 13. Yearly temperature profile of Black Mountain, near Eagle Lake, an abandoned site (elevation $1865 \mathrm{~m}$ ). 


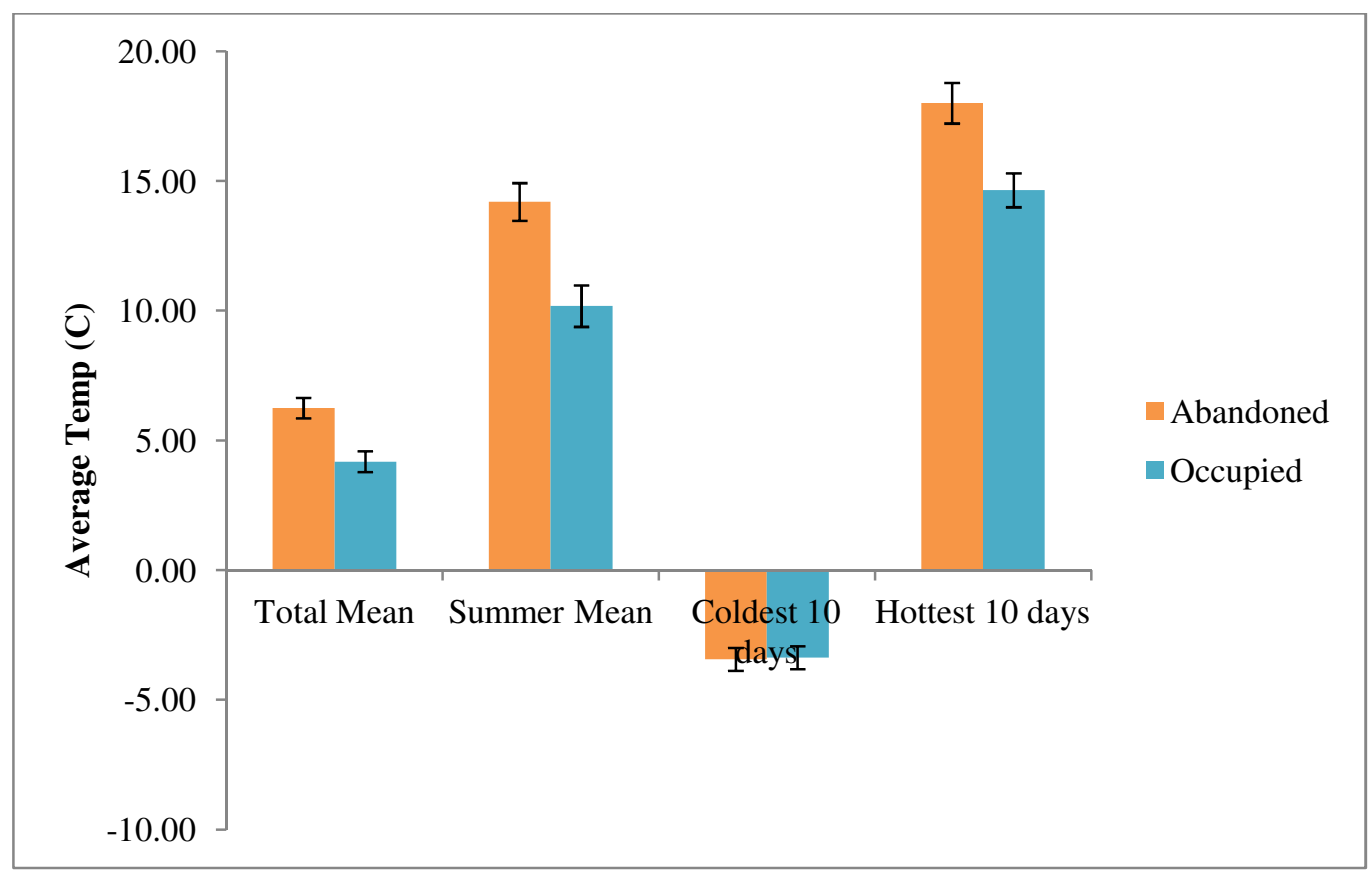

Figure 14. Average temperatures $( \pm \mathrm{SE})$ in abandoned and occupied sites, measured by thermal dataloggers approximately $1 \mathrm{~m}$ in talus matrix in 2009 and 2010 . The coldest 10 and hottest 10 days are the coldest and warmest 10 consecutive days from the study. Summer mean is the average temperature over the calendar days of summer during dates of datalogger deployment. These four microclimate variables represent possible measures of chronic thermal stress in pikas. 


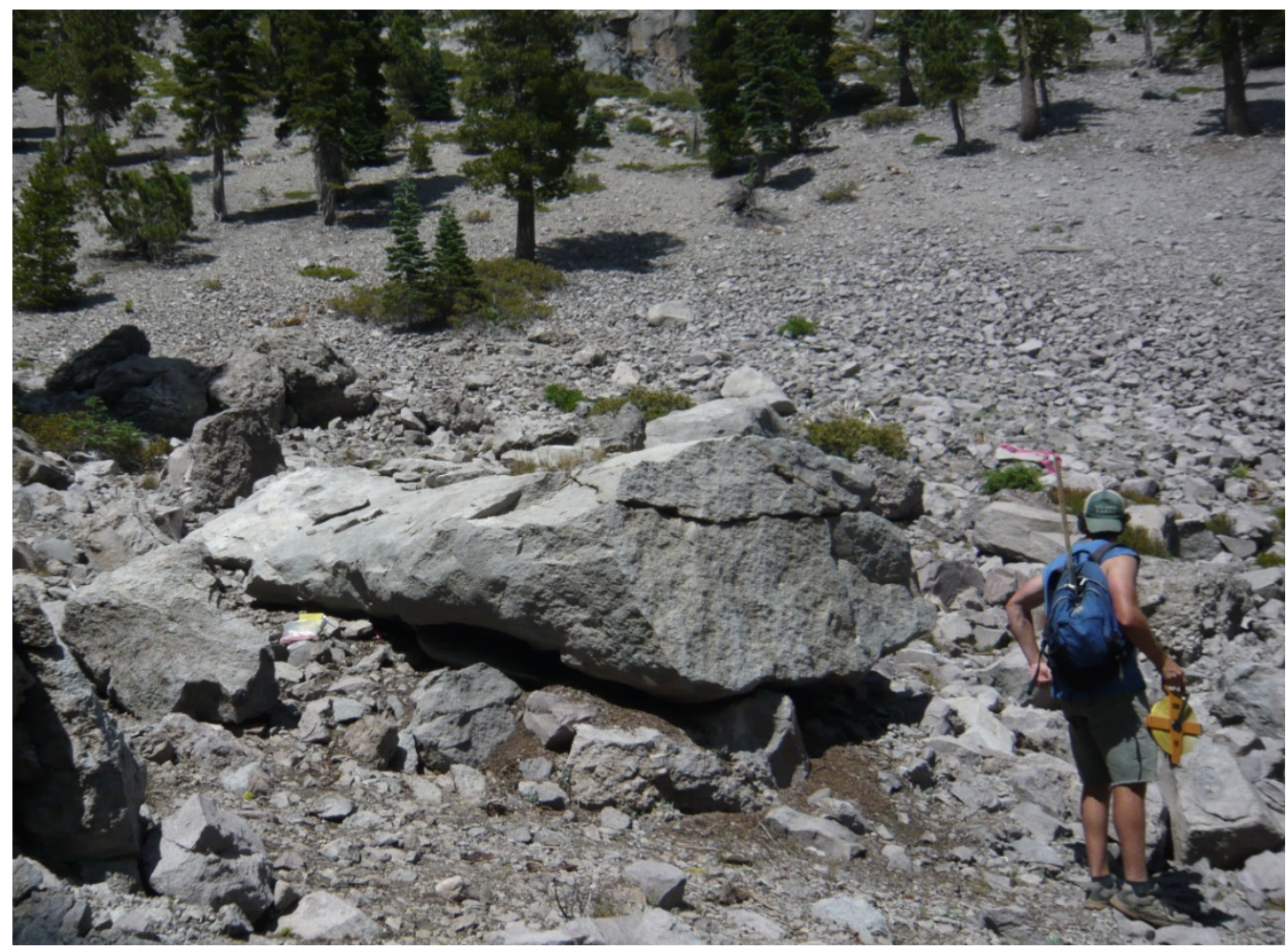

Figure 15. Mosquito pond datalogger site. The datalogger was deployed under the large boulder in the foreground, directly in a haypile. This deployment site is atypical, being more nearly on the surface than within talus infrastructure, and it may explain why this datalogger recorded such high temperatures. The brown material spilling out from under the boulder consists primarily of pika pellets. 


\section{CHAPTER 2. LIST OF REFERENCES}

Beever, E.A. 2002. Persistence of pikas in two low-elevation national monuments in the western United States. Park Science 21:23-29.

Beever, E.A., P.F. Brussard, and J. Berger. 2003. Patterns of extirpation among isolated populations of pikas (Ochotona princeps) in the Great Basin. Journal of Mammalogy 84:37-54.

Beever, E.A., J.L. Wilkening, D.E. McIvor, S.S. Weber, and P.F. Brussard. 2008. American pikas (Ochotona princeps) in northwestern Nevada: a newly discovered population at a low-elevation site. Western North American Naturalist 68:8-14.

Beever, E.A., C. Ray, P.W. Mote, J.L. Wilkening. 2010. Testing alternative methods of climate-mediated extirpations. Ecological Society of America 20:164-178.

Beniston, M. 2003. Climatic change in mountain regions: a review of possible impacts. Climatic Change 59:5-31.

Dearing, M.D. 1995. Factors governing diet selection in a herbivorous mammal, the North American Pika, Ochotona princeps, Dissertation. University of Utah, Salt Lake City, Utah, USA.

Dearing, M.D. 1997a. The manipulation of plant toxins by a food-hoarding herbivore, Ochotona princeps. Ecology 78:774-781.

Dearing, M.D. 1997b. The function of haypiles of pikas (Ochotona princeps). Journal of Mammalogy 78:1156-1163.

Erb, L.P., C. Ray and R. Guralnik. 2011. On the generality of a climate-mediated shift in the distribution of the American pika (Ochotona princeps). Ecology 92:1730-1735.

Franken, R.J. and D.S. Hik. 2004. Influence of habitat quality, patch size and connectivity on colonization and extinction dynamics of collared pikas Ochotona collaris. Journal of Animal Ecology 73:889-896.

Frase, B.A. and W.E. Sera. 1993. Comparison between plant species in bushy-tailed woodrat middens and in the habitat. Great Basin Naturalist 53:373-378.

Grinnell, J., J. Dixon, and J.M. Linsdale. 1930. Vertebrate natural history of a section of northern California through the Lassen Peak Region. University of California Press. Berkeley, California. 
Gustafson, J.R. and R.J. Logsdon. 2007 (Dec 21) Evaluation of petition from Center of Biological Diversity to list American Pika (Ochotona princeps) as threatened California Department of Fish and Game, Wildlife Branch, Sacramento, CA. Nongame Wildlife Program Report 2007-05, 19 pp.

http://www.dfg.ca.gov/wildlife/species/publications/docs/PikaEvaluationReportDec2107. pdf

Hafner, D.J. 1993. North American pika (Ochotona princeps) as a late Quaternary biogeographic indicator species. Quaternary Research 39:373-380.

Hanski, I. 1994. A practical model of metapopulation dynamics. Journal of Animal Ecology 63:151-162.

Hosmer, D.W. and S. Lemeshow. 2000. Applied logistic regression, $2^{\text {nd }}$ eds. New York, NY: John Wiley and Sons, Inc.

Huntly, N.J. 1987. Influences of refuging consumers (pikas: Ochotona princeps) on subalpine meadow vegetation. Ecology 28: 274-283.

Huntly, N.J., A.T. Smith, and B.L. Ivins. 1986. Foraging behavior of the pika (Ochotona princeps) with comparisons of grazing versus haying. Journal of Mammalogy 67:139148.

Intergovernmental Panel on Climate Change (IPCC), 2007. Climate change impacts, adaptation, and vulnerability. In: Contribution of Working Group II to the Fourth Assessment Report of the IPCC. Cambridge University Press, Cambridge, UK.

Inouye, D.W. and A.D. McGuire. 1991. Effects of snowpack on timing and abundance of flowering in Delphinium nelsonnii (Ranunculaceae): implications for climate change. American Journal of Botany 70:997-1001.

Krear, H.R. 1965. An ecological and ethological study of the pika (Ochotona princeps saxatilis bangs) in the front range of Colorado. Ph.D. Thesis. University of Colorado, Boulder.

Lenoir, J., J.C. Gégout, P.A. Marquet, P. de Ruffray, and H. Brisse. 2008. A significant upward shift in plant species optimum elevation during the $20^{\text {th }}$ century. Science 320:1768-1770.

MacArthur, R.A. and L.C.H. Wang. 1973. Physiology of thermoregulation in the pika, Ochotona princeps. Canadian Journal of Zoology 51:11-16.

MacArthur, R.A. and L.C.H. Wang. 1974. Behavioral thermoregulation in the pika, Ochotona princeps: a field study using radiotelemetry. Canadian Journal of Zoology 52:353-358. 
McLaughlin, J.F., J.J. Hellmann, C.L.Boggs, and P.R. Ehrlich. 2002. Climate change hastens population extinctions. Proceedings of the National Academy of Sciences (USA) 99:6070-6074.

Millar, C. USDA Forest Service. Pika Survey Instruction form (online). Accessed Jan. 3, 2011 at http://www.fs.fed.us/psw/publications/millar/PikaSurvey_Instructions_Form.pdf

Millar, C.I. and R.D. Westfall. 2010a. Distribution and climatic relationships of the American pika (Ochotona princeps) in the Sierra Nevada and western Great Basin, USA; periglacial landforms as refugia in warming climates. Arctic, Antarctic, and Alpine Research 42:76-88.

Millar, C.I. and R.D. Westfall. 2010b. Distribution and climatic relationships of the American pika (Ochotona princeps) in the Sierra Nevada and western Great Basin, USA; periglacial landforms as refugia in warming climates. Reply. Arctic, Antarctic, and Alpine Research 42:493-496.

Millar, J.S. and F.C. Zwickel. 1972. Characteristics and ecological significance of hay piles of pikas. Mammalia 36: 657-667.

Moilanen, A., A.T. Smith and I. Hanski. 1998. Long-term dynamics in a metapopulation of the American pika. The American Naturalist 152:530-542.

Moritz, C. 2007. Final Report: A re-survey of the historic Grinnell-Storer vertebrate transect in Yosemite National Park, California. Sierra Nevada Network Inventory \& Monitoring Program, Sequoia \& Kings Canyon National Parks, 47050 Generals Highway, Three Rivers, CA 93271. http://mvz.berkeley.edu/Grinnell/pdf/2007_Yosemite_report.pdf

Museum of Vertebrate Zoology, U.C. Berkeley. Grinnell Resurvey Project (online). Accessed May 9, 2009 at http://mvz.berkeley.edu/Grinnell/index.html

Naftz, D.L., D.D. Susong, P.F. Schuster, L.D. Cecil, M.D. Dettinger, R.L. Michel, and C. Kendall. 2002. Ice core evidence of rapid air temperature increases since 1960 in alpine areas of the Wind River Range, Wyoming, United States. Journal of Geophysical Research 107:10-29.

National Biological Information Infrastructure (NBII). NBII Great Basin. Accessed on August 26, 2011 at http://www.nbii.gov/portal/server.pt/community/great_basin/561.

Nichols, L.B. 2010. Fecal pellets in American pikas (Ochotona princeps) provide a crude chronometer for dating patch occupancy. Western North American Naturalist 70:500507. 
Parmesan, C. and G. Yohe. 2003. A globally coherent fingerprint of climate impacts across natural systems. Nature 421:37-42.

Peacock, M.M. 1997. Determining natal dispersal patterns in a population of North American pikas (Ochotona princeps) using direct mark-resight and indirect genetic methods. Behavioral Ecology 3:340-350.

Quanstrom, W.R. 1971. Behaviour of Richardson's ground squirrel Spermophilis richardsonii richardsonii. Animal Behaviour 19:646-652.

Ray, C. 2006. 2006 Project Report: Testing hypothesized links between climate change and the decline of the American pika. MFWP Scientific Collector's Permit: \#1507. http://www.bristlecone.org/PDFs/pikaproject.pdf

Ray, C., Beever, E., 2007. Distribution and abundance of the American Pika (Ochotona princeps) within Lava Beds National Monument. National Park Service Report, 1-57.

Roach, W.J., N. Huntly, and R. Inouye. 2001. Talus fragmentation mitigates the effects of pikas, Ochotona princeps, on high alpine meadows. Oikos 92:315-324.

Rodhouse, T.J., E.A. Beever, L.K Garrett, K.M. Irvine, M.R. Jeffress, M. Munts, and C. Ray. 2010. Distribution of American pikas in a low-elevation lava landscape: conservation implications from the range periphery. Journal of Mammalogy 91:12871299.

Schmid-Holmes, S., L.C. Drickamer, A. Sessions Robinson. L.L. Gillie. 2001. Burrows and burrow-cleaning behavior of house mice (Mus musculus domesticus). American Midland Naturalist 146:53-62.

Severaid, J.H. 1955. Natural history of the pikas (Mammalian genus Ochotona). Ph.D. Thesis. University of California, Berkeley.

Shardlow, M. R., J. Apel, L.K. Garrett, G. Holm, D. Larson, N. Nordensten, and T. J. Rodhouse. 2009. Upper Columbia Basin Network American pika monitoring protocol: Narrative Version 1.0. Natural Resource Report NPS/UCBN/NRR—2009/XXX. National Park Service, Fort Collins, Colorado.

Shipley, L.A., T.B. Davila, N.J. Thines, and B.A. Elias. 2006. Nutritional requirements and diet choices of the pygmy rabbit (Brachylagus idahoensis): a sagebrush specialist. Journal of Chemical Ecology 32:2455-2474.

Smith, A.T. 1974a. The distribution and dispersal of pikas: consequences of insular population structure. Ecology 55:1112-1119.

Smith, A.T. 1974b. The distribution and dispersal of pikas: influences of behavior and climate. Ecology 55:1368-1376. 
Smith, A.T. and M.L. Weston. 1990. Mammalian Species, Ochotona princeps. Journal of Mammalogy 352:1-8.

United States Fish and Wildlife Service. Department of the Interior Federal Register Notice. May 15, 2008. Determination of threatened status for the polar bear (Ursus maritimus) throughout its range; Final Rule. http://alaska.fws.gov/fisheries/mmm/polarbear/pdf/Polar_Bear_Final_Rule.pdf

United States Fish and Wildlife Service. Department of the Interior Federal Register Notice. February 25, 2010. 12-month finding on a petition to list the American pika as threatened or endangered. http://www.fws.gov/mountain-prairie/species/mammals /americanpika/

Van Vuren, D. and K.B. Armitage. 1991. Duration of snow cover and its influence on life-history variation in yellow-bellied marmots. Canadian Journal of Zoology 69:17551758.

Walther, G.-R., E. Post, P. Convey, A. Menzel, C. Parmesan, T.J.C. Beebee, J.-M. Fromentin, O. Hoegh-Guldberg, and F. Bairlein. 2002. Ecological responses to recent climate change. Nature 416:389-395.

Walther, G.-R., S. Beissner, and C.A. Burga. 2005. Trends in the upward shift of alpine plants. Journal of Vegetation Science 16:541-548.

Western Regional Climate Center. Northern California Climate Summaries (online). Accessed January, 212012 at http://www.wrcc.dri.edu/summary/climsmnca. htmlhttp://www.wrcc.dri.edu/summary/climsmnca.html

Wilkening, J.L., C. Ray, E.A. Beever, and P.F. Brussard. 2011. Modeling contemporary range retraction in Great Basin pikas (Ochotona princeps) using data on microclimate and microhabitat. Quaternary International 235:77-88.

Wilson, R.J., D. Gutiérrez, J. Gutiérrez, D. Martinez, R. Agudo, and V.J. Monserrat. 2005. Changes to the elevational limits and extent of species ranges associated with climate change. Ecology letters 8:1138-1146.

Wolf, S. 2010. Distribution and climatic relationships of the American pika (Ochotona princeps) in the Sierra Nevada and western Great Basin, USA; periglacial landforms as refugia in warming climates. Comment. Arctic, Antarctic, and Alpine Research 42:490492.

Wolf, S., B. Nowicki, and K. Siegel. 2007a. Before the California Fish and Game Commission: Petition to list the American pika (Ochotona princeps) as threatened under the California Endangered Species Act. Center for Biological Diversity. http://www.biologicaldiversity.org/species/mammals/American_pika/pdfs/petition_ca2007.pdf 
Wolf, S., B. Nowicki, and K. Siegel. 2007b. Before the Secretary of the Interior: Petition to list the American pika (Ochotona princeps) as threatened or endangered under the United States Endangered Species Act. Center for Biological Diversity.

http://www.biologicaldiversity.org/species/mammals/American_pika/pdfs/Americanpika-federal-petition-10-01-2007.pdf 


\section{CHAPTER 3.}

\section{$\underline{\text { Activity Profiles of High and Low Elevation }}$}

\section{$\underline{\text { Pikas in the Sierra Nevada }}$}

\section{INTRODUCTION}

The earth is expected to warm $1-8^{\circ} \mathrm{C}$ in the next century (A1B:SRES scenario, IPCC 2007). This is likely to have profound effects on organisms around the world. In the last 100 years the earth has warmed $0.6^{\circ} \mathrm{C}$, and this is already having a measurable effect on many organisms (Walther et al. 2002). Increasing amounts of evidence suggest the phenology of animal and plant activity is being influenced by climate change (IPCC 2007, Parmesan and Yohe 2003). Spring activities, such as the breeding of birds and amphibians and the flowering of plants, have occurred earlier and earlier over the last 50 years (IPCC 2007, Parmesan and Yohe 2003). As temperatures increase, some animals are moving northward (in the northern hemisphere) and montane animals are moving upward in elevation (IPCC 2007, Parmesan and Yohe 2003). Montane organisms are at particular risk, not only because optimal habitat is found at increasingly higher altitude, but also because temperatures are predicted to rise more rapidly at higher elevations (Beniston 2003, Naftz et al. 2002).

The American pika, Ochotona princeps, is a small (121 - $176 \mathrm{~g})$ lagomorph that lives in talus slopes or other types of broken rock in mountainous areas in western North America (Smith and Weston 1990). In the southern part of their range American pikas usually occur at elevations over $2500 \mathrm{~m}$ (Smith and Weston 1990). Pikas are 
herbivorous, eating a variety of grasses, sedges, and leafy vegetation (Smith and Weston 1990). They do not hibernate during the long montane winter; instead they survive on stores of vegetation, or haypiles, stockpiled during the summer and autumn (Smith and Weston 1990). Because areas of talus tend to be patchily distributed over a landscape, they effectively act as islands, separating pika demes from each other (Moilanen et al. 1998). In fact, pikas are considered to have a classic metapopulation structure (Moilanen et al. 1998). They are highly reliant on patches of talus, which are separated by meadows, and they rarely move more than six meters from the edge of the talus to forage (Franken and Hik 2004, Huntly et al. 1986, Roach et al. 2001). There is only limited dispersal among patches: about $25 \%$ of juveniles attempt to disperse from their place of birth (Smith and Weston 1990). Juveniles can apparently disperse only very short distances, likely because they are more susceptible to predation the longer they are away from talus. In fact, distances of over $300 \mathrm{~m}$ have been found to act as barriers for dispersing juveniles (Smith 1974a). However, there is genetic evidence that pikas may be able to disperse as far as $2 \mathrm{~km}$ in some areas (Peacock 1997). Even so, the further apart these patches of talus, the less chance there is of dispersal between patches (Franken and Hik 2004, Smith 1974a). This could have serious implications in regard to climate change because, if habitat conditions deteriorate, pikas would be unable to relocate long distances to find more suitable conditions.

The American pika has gained recent attention as a possible indicator species of climate change (Jeffress et al. 2010), because of both their alpine obligate status and their low thermal tolerance. They rely on talus not only for protection from predators, but also for thermal cover. Studies (MacArthur and Wang 1973, Smith 1974b) show that pikas 
will die if confined aboveground at temperatures as low as $25.5^{\circ}-28^{\circ} \mathrm{C}$. This inability to regulate body temperature during heat is perhaps the trait that makes pikas most vulnerable to climate change. It puts lower elevation pikas, particularly those in the southern portion of their range, at elevated risk of overheating. If atmospheric temperatures increase greatly, it is possible that the shade temperatures of the talus could go above the lethal limit for pikas. In addition, higher temperatures could decrease the amount of time pikas can spend foraging, preventing them from gaining sufficient body mass and from collecting enough hay for overwintering (Beever et al. 2003, Wilkening et al. 2011). Due to the pika's thermal sensitivity and the current climate predictions, American pikas have been proposed for listing under the Endangered Species Act, both in California (Wolf et al. 2007a) and federally (Wolf et al. 2007b).

There is some evidence to suggest that pikas may be more adaptable to climate change than people have suspected. American pikas apparently seek out thermal refugia, such as rock-ice features (Millar and Westfall 2010) and lava formations (Beever 2002, Rodhouse et al. 2010), in which colder air and even year-round ice is trapped in the talus infrastructure. Populations of American pikas in different temperature regimes may also exhibit behavioral plasticity, or have genetic differences, such that these populations do not have the same activity schedules. Pikas cannot regulate their body temperature to warm temperatures physiologically, so they must regulate behaviorally (MacArthur and Wang 1974, Smith 1974b). They tend to remain under talus and are less active on warmer days (MacArthur and Wang 1974, Smith 1974b). In lower elevation sites they may come out of their rock burrows primarily during the morning and evening hours, whereas in higher elevation sites they may remain active throughout the day (Smith 
1974b). This general tendency has been observed and noted by many researchers, but has only been field tested in one study involving two sites, one low $(2550 \mathrm{~m})$ and one high (3400 m) elevation (Smith 1974b). In the current study I investigated whether this pattern occurs in other sites in the Sierra Nevada, by observing and comparing activity rates of pikas at one low and one high elevation site of similar elevations to Smith (1974b). However, I selected a more natural low elevation population. The low elevation pikas that Smith (1974b) studied inhabited mine tailings, man-made rock piles of artificial habitat, in the former mining towns of Bodie, Masonic, and Aurora, California.

Because lower elevation pikas are likely to experience warmer temperatures, they may be constrained in the hours of the day they can be active, and they may be more active at dawn and dusk than higher elevation pikas. Smith (1974b) found that pikas in his low elevation site exhibited a more crepuscular activity schedule than high elevation pikas. Crepuscular behavior, particularly if found to a greater extent at the low elevation site, may indicate that pikas are demonstrating behavioral plasticity between sites, and may have at least limited ability to adapt to rising global temperatures. Whether or not this plasticity could result in evolution, or if it could be the result of genetic differences between the populations, was beyond the scope of this project, but these preliminary investigations could help set the stage for future research. Additionally, if low elevation pikas are becoming less diurnal, the next logical question is, could they be nocturnally active? If pikas are active at night, they would not be so limited in the amount of time they could forage and collect hay. Currently, almost nothing is known about the nighttime behavior of the American pika, but laboratory studies suggest that another 
species of pika, the Afghan pika (O. rufescens rufescens) is nocturnal (Kosaka et al. 1988). American pikas have long been considered diurnal, yet their nighttime behavior is significantly understudied. Many researchers report hearing pikas call at night (Krear 1965, Smith 1974b, C. Ray pers. comm., pers. obs.), but the significance behind nocturnal alarm calling has never been investigated. As a preliminary study on nocturnal behavior in American pikas, I experimented with the use of infra-red remote cameras, to determine if this is a feasible method for investigating nighttime activity in pikas.

I sampled in August and September, and compared diurnal pika activity rates to determine if there were seasonal and time-of-day differences between the two sites, and if these differences were related to temperature. I had several predictions regarding pika activity levels among the sites. (1) I expected lower activity rates to correspond with higher temperatures, and vice versa. (2) I expected the low elevation population to exhibit a more crepuscular activity schedule than the high elevation population. (3) Aside from the effects of temperature on pika behavior, I expected to find seasonal differences in activity rates between the sites. Specifically, I expected to see less haying behavior in the low elevation site in September. Smith (1974b) recorded that haying stopped six weeks earlier in his low elevation site, while pikas in the high elevation site went on haying through September. I expected foraging activity rates to stay the same throughout the study as pikas met their daily energy requirements. 


\section{METHODS}

\section{Study Area}

This study was conducted in the Sierra Nevada Mountains of California in August and September 2010, when pikas actively make their haypiles (Kawamichi 1976). One low and one high elevation site were selected from the Bishop Creek drainage system in central California, on the eastern side of the Sierra, approximately 20 miles west of the town of Bishop (Figure 1). The two sites were $4.9 \mathrm{~km}$ apart, which is beyond the reported upper dispersal limit of pikas (2 km, Peacock 1997). Based on the distance apart, pikas in the two sites were assumed to be distinct populations.

Low elevation plots ranged from $2493-2835 \mathrm{~m}$ in elevation (mean $\pm \mathrm{SE}=2616 \pm$ $33.68 \mathrm{~m}$ ) and were located near the community of Aspendell, California in the Inyo National Forest $\left(37^{\circ} 13-15^{\prime} \mathrm{N}, 118^{\circ} 34-35^{\prime} \mathrm{W}\right)$. The slopes in the low site were eastnortheast and west-northwest facing (aspects of $59-61^{\circ}$ and $290-323^{\circ}$ ). The dominant vegetation surrounding talus consisted of aspen (Populus tremuloides) and a variety of shrubs, including sagebrush (Artemisia tridentata) and fernbush (Chamaebatiaria millefolium). A low percentage of forbs and graminoids appeared to be growing in close vicinity to the talus. The low elevation site is an atypical representation of pika habitat, and could be considered low quality, based on its lack of forbs (Rodhouse et al. 2010, Wilkening et al. 2011), and higher percentage of shrubs (Lassen Chapter).

The high elevation plots were at 3291-3389 $\mathrm{m}$ in elevation (mean $\pm \mathrm{SE}=3321 \pm$ $10.05 \mathrm{~m})$, on slopes with north and northeast aspects $\left(355-358^{\circ}\right.$ and $\left.26-59^{\circ}\right)$. This site was located near Loch Leven and Piute Lakes in the Inyo National Forest (37 $13-14^{\prime} N$, 
$\left.118^{\circ} 39-40^{\prime} W\right)$. Dominant vegetation consisted of typical Sierra Nevada alpine meadow vegetation, including a variety of sedges (Carex spp.), and columbine (Aquilegia spp.). Forbs and graminoids were widely available and utilized by pikas, as determined by analysis of haypile composition as well as behavior observations over the course of the study. The high elevation site was chosen for its "typical" pika habitat: talus slopes next to alpine meadows (Smith and Weston 1990). I selected more northerly aspects in this site to increase the temperature contrast between the high and low elevation sites.

\section{Sampling Design}

Each site contained five plots (low site numbered 1-5, high site numbered 6-10) (Figure 1). Neighboring plots within each site were 240-570 m apart, with the exception of a pair of neighboring plots that were separated by $140 \mathrm{~m}$ (plots 1 and 3); these plots were divided by a main highway, which pikas were never seen to cross. Each plot contained three subplots (labeled a-c), resulting in a total of 15 subplots in each site, or 30 subplots total, although not all subplots were used in the final analysis (see below). Each subplot used in the analysis contained at least one unique pika, whose territory was believed to center in the subplot (Figure 2). I selected plots and subplots based on the territory of each pika as well as the range of view by the observer, so the sizes and shapes of plots and subplots varied.

Subplots contained 1-3 pikas, and were approximately $150-600 \mathrm{~m}^{2}$, falling within or under the recorded territory size of one pika (400-700 $\mathrm{m}^{2}$, Smith and Weston 1990). Subplots that were under $400 \mathrm{~m}^{2}$ were constrained by vegetation or lesser quality talus, and appeared to envelope the majority of one pika's territory. Pika territory size and 
density (or degree of overlap) appear to be more variable than has been recorded and may depend upon habitat (C. Millar pers. comm., pers. obs.).

I selected observation platforms that gave the best view of subplots and were at least $5 \mathrm{~m}$ away from the focal pika(s) to limit disturbance, but no more than $40 \mathrm{~m}$ to ensure visibility. Most observation platforms allowed the observer to view two to three subplots from the same location, by only a slight shifting of position (e.g. turning around) (Figure 2). This was done to limit disturbance, and to reduce time between samples. Pikas were not always predictable in their movements, and sometimes came within $5 \mathrm{~m}$ of the viewing platform, or ranged far outside the boundary of the subplot. Movements outside of the subplot borders were recorded only anecdotally. If a pika was too close to the viewing platform (within $5 \mathrm{~m}$ ), this was noted on the datasheet, and this pika was not used in analysis during the time it was close to the observer, to eliminate human-biased activities.

Temperatures were recorded automatically every two minutes, using Thermochron iButton temperature recorders (model DS1922L, Embedded Data Systems, Lawrenceburg, KY). The iButtons were protected within ventilated copper capsules and set on the talus surface in the shade immediately upon arrival at a plot. They were left to record for the entire observation period, and were collected upon departure.

\section{Activity Profiles}

The observations were conducted in four two-hour blocks each day (time blocks 1-4). The morning block (time block 1) began half an hour before sunrise, and the evening block (time block 4) ended ten minutes before sunset. These times were selected 
to ensure visibility of pikas. The midday blocks were split evenly in the middle of the day, in an attempt to capture the warmest hours. The time blocks changed between August and September because of diminishing daylight hours. In August, the time blocks were: 6:00-8:00, 10:00-12:00, 14:00-16:00, and 18:00-20:00. In September, the time blocks were: $6: 30-8: 30,10: 00-12: 00,13: 30-15: 30$, and 17:10-19:10. Each subplot was observed once in every time block, both in August and September.

Subplots within each plot were observed in random order (randomized by rolling of a die), for ten to twenty minutes at a time. Most subplots could be observed from the same observation platform, so those that were observed first were always observed for 20 minutes, and those observed second and third were observed for 10 to 20 minutes, depending on the time available. Observations began upon arrival at an observation platform, as soon as the observer had located or attempted to locate pikas within the subplot. After a brief acclimation time pikas appear to become accustomed to human presence (Hayes and Huntly 2005, Holmes 1991, Huntly et al. 1986, Ivins and Smith 1983, Lutton 1975, Roach et al. 2001). I analyzed the data later to determine if an acclimation period was necessary, so that the acclimation time could be removed if it had a different activity rate.

Observations were scan samples performed every 30 seconds, timed using a stopwatch. Every time a pika was seen above ground, the observer recorded the behavior of the pika in the appropriate 30-second time slot (labeled on the data sheet). Observers recorded all behaviors seen throughout each 30-second time slot. Behaviors recorded were: haying, foraging, perching, grooming, visibly calling, and running (see Table 1 for definitions). Other behaviors were noted and described. Repeated occurrences of the 
same behavior were not recorded within the same 30-second time slot (for each individual pika). When no pikas were observed aboveground, the observer marked the "inactive" box to ensure that there was a record for every 30 -second time slot. If the pika was known to be active aboveground but could not be seen (e.g. was behind a bush), the column labeled "out of view" was marked. Observers used their best judgment, because when a pika was out of view, it was more often underground than aboveground. However, because of physical barriers in some plots, pikas could not always be seen when they were active, but occasionally their presence was revealed by the sound of foraging or a visibly shaking bush.

Pikas in each subplot were identified by physical characteristics, such as scars on and size of ears, pelage coloration, and molting lines. Other researchers have used similar physical characteristics to identify individual pikas (Barash 1973, Kawamichi 1976, Lutton 1975, Tyser 1980). Observers in this study made every effort to keep track of individuals and to identify them when possible, and all pikas seen in one subplot during an observation session were recorded and given unique IDs. In each observation session, observers recorded the total number of pikas observed, which helped to determine the total number of pikas in each subplot. Determining the total number of pikas per subplot was necessary for comparing the activity levels at each site (see Data Analysis). All final estimates of subplot occupancy were conservative (e.g. if one sample had four pikas, but the rest had three, the subplot was given an occupancy of three, with the assumption that the fourth animal had been an infrequent visitor. Activities of the "visitor" were then not included in analysis. Observers attempted to identify the visitor and residents based on physical characteristics). 


\section{Data Analysis}

\section{Temperature}

I averaged temperatures recorded by iButtons for each observation period. When observers changed locations, the iButton usually took a minute or two to equilibrate, so the first record was never used in the average. Occasionally, the iButton took longer to equilibrate, as evidenced by a much higher temperature in the beginning of the sample period. In all instances, only equilibrated temperatures (the temperature had reached a steady value) were used.

\section{Activity Rates}

I defined "aboveground activity" as all activities in which the pika was visible, i.e., all activities, except calling when the pika was not seen calling. To calculate aboveground activity rate (hereafter, activity rate), the activities from each observation session had to be condensed. When any aboveground activity occurred in one 30 -second time slot, a value of 1 was given. If one pika did multiple activities in a 30 -second time slot, the aboveground activity was still given a value of 1 , to give equal weight no matter the actions of the pika. For instance, a pika that perched, groomed, and visually called in one 30 -second time slot was considered just as active as a pika that ran in one 30 -second time slot. If two pikas were active aboveground in one time slot, the aboveground activity was given a value of 2 , and so on for multiple pikas. Aboveground activity was summed for the entire observation session, and this value was normalized by the total number of pikas in each subplot, so that subplots with more pikas did not appear to have higher activity rates. To calculate activity rate, this normalized activity value was divided by the number of 30 -second time slots of observation. Time slots that the pikas 
were active but out of view were removed from analysis. Therefore, the highest activity rate possible for a subplot containing only one pika would be $40 \div 1 \div 40$, or 1 , for a pika that was visible in every time slot for 20 minutes (= 40 time slots). I calculated the activity rates of specific activities (e.g., foraging) the same way as the aboveground activity rate, except that I summed only the occurrences of that specific activity.

Prior to all analyses involving specific and aboveground activity rates, all types of activity rates were $\operatorname{logit}$-transformed $(\log (y /[1-y])$, where $y=$ activity rate $)$ to reduce skew in the data. Because the dataset contained activity rates of both 0 and 1 , I added 0.01 (the largest non-zero activity rate) to both the numerator and denominator, as suggested by Warten and Hui (2011). All statistical analyses were conducted in R 2.12.0 (C2010 The R Foundation for Statistical Computing, http://www.r-project.org/foundation/).

\section{Acclimation Time}

Before all of the data could be used in the overall analysis, I had to determine whether a behavioral acclimation period was necessary for the pikas to adjust to human presence. Each sampling period that included an acclimation time was separated into three segments: first 5 minutes, second 5 minutes, and final 10 minutes. I then compared the activity rates in these time segments using a general linear mixed model (GLMM) (Crawley 2007) with a significance of alpha $=0.05$. I treated activity rate as the

dependent variable, and the acclimation time segments as fixed explanatory variables. To account for random differences between plots, I included plot as a random effect. If I determined that no acclimation was necessary (i.e., the time segments did not have significant differences in average activity rate), I pooled the time segments into one sample. If I determined that an acclimation period was necessary (average activity rates 
were significantly different among time segments), I removed those segments that differed significantly from the final segment and did not use them in the final analysis.

\section{Activity Analysis}

After adjusting for acclimation, I developed a GLMM to determine if activity rate was different at different temperatures and between sites, time blocks, and months, once again treating subplots and plots as random effects, and nesting subplots within plots. I used a significance of alpha $=0.1$, with activity rate as the dependent variable, and site, time block, and month as the fixed explanatory variables. I ran models using both additive and interaction effects, beginning with the most complicated model (testing an interaction of site, month, and time block, with the additive effect of temperature). I then simplified the model by removing interactions when interactions were not significant, but retained all variables to account for their fixed or random effects. To check for differences in temperature between sites, months, and time blocks I also ran a GLMM treating temperature as the dependent variable, and sites, time blocks, and months as the fixed explanatory variables, using a significance of alpha $=0.1$. Once again, I nested subplots within plots and treated these as random effects. I checked to see if daily temperature trends were different between the sites by testing the interaction of site and time block.

Plots were assumed to be independent because they were far enough apart to greatly diminish the chance of recording the same pika. It is unlikely that any individual pika moved $200 \mathrm{~m}$ or more to another plot during the sampling period. Observation sessions were typically carried out simultaneously by two people in neighboring plots, 
and pikas were never seen running from one plot to another, or traveling more than $60 \mathrm{~m}$ in any direction.

\section{Remote Camera Placement}

To document pika activity I installed four infrared digital game cameras (GameSpy I45, Moultrie Products, Alabaster, AL) in two plots in the low elevation site for two weeks in October, 2010. I attached each camera to a three foot mounting post that was stabilized in talus crevices by moving loose rocks. I used different camera settings, but most often I used the multi-shot option of three still pictures taken per detection, with a minimum delay between picture sets of one minute. The cameras were set to give the date, time, and temperature in degrees Celsius for each picture taken, and the infrared flash was set to "on". To prevent saturation from the flash, I used masking tape to cover various portions of the flashbulbs.

I experimented with placement of cameras and checked them every one to three days for results. I placed the cameras 10 to $60 \mathrm{~cm}$ above the talus, and 0.5 to $10 \mathrm{~m}$ from haypiles and known high use areas. Cameras were sometimes placed on opposite sides of the same haypile to check for accuracy in activity determination. To ensure that the cameras were pointed in the correct area, I purposely triggered them myself and checked the results until the placement was as desired. When I returned, I collected memory cards and viewed the pictures taken, counting the number of pika pictures obtained, and calculating each camera's success rate (percentage of pictures that were of pikas). 


\section{RESULTS}

\section{Activity Profiles}

\section{Observations}

In total, two assistants and I conducted 4049.5 minutes of observations (Table 2). Pikas were seen aboveground in $25 \%$ of the 30 -second time slots, but $64 \%$ of observation periods had visual observations at some point during the session. We observed a total of 49 pikas in August (low site, 22; high site, 27) and 41 in September (low site, 16; high

site, 25) (Table 2). In two subplots, one in September (5B) and one in both months (2A), no pikas were observed (or calls heard) during any observation period, so these subplots were removed from the analysis, during the time they contained no pikas.

Each subplot was observed once in every time block in both August and September. I removed two sampling periods from the analysis because of the presence of predators, a fisher (Martes pinnanti) and an American marten (M. americana), hunting in the talus. In each case, the pikas hid under the talus for the duration of the sampling period after the predator had been detected. Both of these events occurred in August, in the high elevation site, during the sunset time block.

\section{Temperature}

During observations, shade temperatures on the talus surface ranged from $2.79-$ $33.03^{\circ} \mathrm{C}$ in the low elevation site, and from $5.50-26.95^{\circ} \mathrm{C}$ in the high elevation site. The average temperatures of the sites in August and September are given in Table 3. Temperature was significantly higher in August than September $\left(\mathrm{F}_{1,198}=22.2141, P<\right.$ $0.0001)$ and it varied throughout the day $\left(\mathrm{F}_{3,196}=171.0739, P<0.0001\right)$ (Figure 3). Time 
block 3 was the warmest period of the day, and time block 1 the coolest (Figure 3 ). The low elevation site was warmer than the high elevation site $\left(\mathrm{F}_{1,8}=4.3265, P=0.0711\right)$. Temperature varied significantly between the sites at different times of day (interaction between sites and time block: $\left.\mathrm{F}_{3,193}=12.8757, P<0.0001\right)$.

\section{Acclimation Time}

I retained all minutes of observations because no acclimation was deemed necessary for pikas to adjust to human presence. There was no significant difference in activity rate between the first five minutes, the second five minutes, or the final ten minutes of observations $\left(\mathrm{F}_{2,456}=1.5577, P=0.2117\right)$ (Figure 4). Additionally, when I compared all samples that had an acclimation period to samples that did not, pika activity rates were not significantly different $\left(\mathrm{F}_{1,217}=0.0032, P=0.9551\right)$ (Figure 5).

\section{Activity Rates}

The average aboveground activity rates of pikas in both sites in August and September are given in Table 4. Pikas were more active early and late in the day (Figure 6). After accounting for the effects of temperature and month, activity rates were significantly different between the sites at different time blocks ( $\mathrm{DF}=3,191, P=$ $0.0123)$.

Other interactions were not significant in predicting activity rate (Table 5). Additionally, neither temperature or month was significant, once the interaction of site and time block was accounted for (Table 5). Although the interaction between site and month was not significant, the low site did have an increased activity rate in September, while the high site maintained approximately the same activity level between months (Figure 7). 


\section{Rates of Specific Activities}

The most common aboveground behavior observed in both sites was "perching", closely followed by "running" (Figure 8). Significantly more foraging occurred in the high elevation site than the low elevation site $(\mathrm{DF}=1,8, P=0.0104)$ (Table 6 , Figure 8$)$. We observed the highest rates of foraging in the morning, and foraging rates decreased throughout the day in both sites (Figure 9).

Haying was the only behavior with seasonal differences between high and low sites (interaction of site and month) $(\mathrm{DF}=1,193, P=0.0539)$ (Table 7). In the low site, pikas hayed more frequently in the month of August, and in the high site, pikas hayed more frequently during September (Figure 10).

Daily perching and running rates varied between the high and low elevation sites (interaction of site and time block) (Tables 8 and 9). We observed pikas running and perching more frequently in the morning and evening hours in both sites (Figures 11 and 12). In the high elevation sites, pikas ran and perched more frequently during the midmorning time block than they did in the low sites. Grooming rates apparently were not significantly affected by temperature, site, month, or time block (Table 10).

\section{Remote Cameras}

I tested cameras at varying distances from haypiles and activity zones and found that only cameras placed at an intermediate distance of 2 to $4.5 \mathrm{~m}$ were successful in capturing pika activity (Figure 13). The camera placed less than one meter from a haypile took ten pictures over the course of 24 hours that were too fuzzy to make out 
(Figure 14). At distances of $10 \mathrm{~m} \mathrm{I} \mathrm{did} \mathrm{not} \mathrm{get} \mathrm{any} \mathrm{pictures} \mathrm{of} \mathrm{pikas,} \mathrm{but} \mathrm{obtained} 0$ to 10 pictures of rocks in the course of 24 hours.

Cameras were successful at two plots, when the camera was 2 to $4.5 \mathrm{~m}$ from the haypile or activity zone and 10 to $30 \mathrm{~cm}$ high (Figures 13 and 15). In the course of 24 hours, the number of pika pictures varied by location. On average, in a 24 hour period, one camera produced 1 pika picture, one produced 5.7, and one produced 21.2. These latter two cameras were on opposite sides of the same haypile. The success rate of the three cameras varied between $18.5 \%$ to $53.2 \%$.

Pictures of pikas were taken throughout the day, from $6: 30$ am to $6: 30 \mathrm{pm}$. A few of the early morning pictures were taken in the dark with the infrared flash. There were no pika pictures taken at night, but several woodrat pictures were taken in the middle of the night, so cameras were functioning during that time. Additionally, most cameras took a few nighttime pictures of rocks (2-6 per night). The flash was very bright, causing night pictures to be washed out, so I experimented with covering portions of it with masking tape. The clearest night pictures resulted from taping over half of the flashbulbs. 


\section{DISCUSSION}

In this study, I confirmed that pikas are most active early and late in the day, but I determined that their diurnal activity schedule is not directly related to temperature. Additionally, I found that pikas may be able to adjust their activity schedules, as pikas in the low elevation site exhibited more crepuscular behavior than pikas in the high elevation site in August. The low elevation pikas escaped higher ambient temperatures by remaining under talus, suggesting that American pikas may have some ability to adapt to rising temperatures. I also found that pikas in the low elevation site foraged less than those at higher elevations, suggesting either adaptation to climate change (through unconfirmed nocturnal foraging), or stress at lower elevations.

\section{Methodology}

Prior to this study, researchers studying pika behavior have used a variety of acclimation times before observation (Barash 1973, Hayes and Huntly 2005, Ivins and Smith 1983, Kawamichi 1976, Lutton 1975, McKechnie et al. 1994, Roach et al. 2001, Svendsen 1979, Tyser 1980). Some researchers have employed a 10 - 15 minute acclimation time, during which no recordings were made (Hayes and Huntly 2005, Lutton 1975), and many apparently used no acclimation time at all (Barash 1973, Ivins and Smith 1983, Kawamichi 1976, Svendsen 1979, Tyser 1980). To date, no one has specifically looked to see how long of an acclimation time pikas require to become accustomed to human presence. In my study area, pikas did not require any acclimation time. There was a slight, but non-significant, decrease in pika activity in the first five 
minutes of observation, followed by a slight, non-significant, increase in activity rate in the next five minutes. A standardized acclimation time, or an agreement that no acclimation time is necessary, would greatly increase the efficiency and accuracy of pika observational studies. I would suggest that no acclimation time is necessary, but to be sure, this finding should be tested over a broader study region and a larger sample size.

Many subplots contained more pikas in August than in September (Table 2), and I believe these "extras" were the new pups of the season that apparently dispersed by the September sampling. Pika pups share the home range of their mothers immediately after weaning, and gradually reduce the home range overlap as the season progresses (Smith and Ivins 1983). The possible juveniles in my sites were slightly smaller, more uniform in color than adults (typically gray), and without molting lines or scars, which matches the description of juvenile pikas (C. Ray pers. comm.). Because I accounted for the monthly occupancy number in each subplot in calculating activity, the change in subplot occupancy size should not affect the overall activity patterns in this study.

\section{Activity Profiles and Relationship to Temperature}

Alterations in environmental conditions caused by climate change are likely to impose strong selection pressures on traits related to survival in organisms around the world. Populations respond to environmental change in three primary ways: by relocating to more suitable habitats, by adjusting their behavior through phenotypic plasticity (without changes in their genetic makeup), and through natural selection resulting in genetic adaptations (Gienapp et al. 2008). As pikas have very limited ability to relocate long distances to find more suitable habitats (Smith 1974a), the latter two 
represent their most likely responses to climate change, assuming environmental changes occur at a slow enough rate to allow adaptation. I expected lower elevation pikas to be exposed to more stressful climatic conditions, and thus to be more likely to exhibit either behavioral plasticity or genetic adaptations in response to these conditions, particularly to higher temperatures. Differences between the populations in response to temperature could initially be caused by phenotypic plasticity, but eventually may lead to evolution by natural selection, and thus genetic adaptation (Price et al. 2003). Pikas in the low elevation site did exhibit a different activity schedule than pikas in the high elevation site, suggesting that they may be responding to warmer temperatures. Pikas in both sites adjusted their activity rates throughout the day, but these adjustments did not appear to be based on temperature. In August, pikas in the low elevation site exhibited a more crepuscular activity schedule than pikas in the high elevation site. This could be a result of a warmer climate (over many generations) in the low elevation site, which suggests the possibility of behavioral plasticity in pikas, or genetic differences between the populations. Rapid changes in behavior in response to specific stimuli are more indicative of plasticity than genetic adaptation (Gross et al. 2010). However, separating phenotypic plasticity from evolutionary responses (genetic adaptations) is very difficult (Gienapp et al. 2008). To determine if pikas in the two populations are exhibiting different genetic adaptations, I would need to test for long-term changes in behavior, for instance by translocating high and low elevation pikas and investigating whether they respond in similar ways to temperature in their new environments. This study has confirmed that there are differences between the two populations of pikas, and future studies can investigate the nature of these differences and what causes them. 
Consistent with my prediction, pikas exhibited significantly different activity rates at different times of day in the high and low elevation sites, as stated above. This difference in activity schedules was significant in the overall study, but if the months are analyzed separately significance is not found in both months (Figure 6). The activity schedule of pikas in the high site in August is puzzling. Compared to the low site, pikas were more active in time block 2 , and less active in time block 4 , which does not correspond to temperature trends (Figure 3). Activity increased in time block 2 when temperatures were warmer in the high site than the low site, and it decreased in time block 4 when temperatures were colder (Figure 3). Because this difference between sites does not appear to be due to temperature, there must be other factors involved. Seasonal influences, such as the shorter growing season at the high elevation site, or predation risk may both be influencing pika activity schedules in the high elevation site. Perhaps there is a greater risk of predation at dusk during the month of August than there is later in the season at the high elevation site. We observed no predators in September, but in August we did see a fisher and an American marten in the high elevation site, both hunting in the talus at dusk. If pika activity levels are influenced by predation risk, these results do not point to behavioral plasticity due to climate. Instead, they reveal a much more complicated picture of what factors dictate pika daily activity schedules. Another field season with the addition of new sites will hopefully help to clarify these results, but a more focused study on pika predation is needed to fully investigate this as a possibility.

Surprisingly, I found that pika activity is not dictated by temperature, once time of day has been accounted for. This is contrary to previous studies that did not include time of day as a covariate in their analyses (MacArthur and Wang 1974, Smith 1974b). It 
appears that pika behavior schedules are at least partially set, and are not adjusted based on temperature. I did not sample during times of precipitation, or in higher wind conditions, but I would guess pikas make exceptions for more drastic weather conditions. Hayes and Huntly (2005), for instance, found pikas to be less active in windier conditions, and many researchers agree that pikas stay below the surface during precipitation events (A. Smith pers. comm.).

Although an altered activity schedule may benefit low elevation pikas by reducing their exposure to high temperatures, reduced activity could have negative consequences as alpine temperatures increase. If pikas are limited in the amount of time they can spend gathering haypiles and foraging due to high temperatures, survival rates and population health could be reduced. Undernourished pikas could be more susceptible to disease and parasites and less reproductively successful. Both reduced summer foraging and lower haypile size could increase pika mortality by reducing a pika's preparedness for winter, although less time spent haying is not necessarily a threat to pika health. The full usefulness of the haypile itself is questionable, and is likely location specific (Millar and Zwickel 1972, Dearing 1997b). Not all pikas collect haypiles (Beever et al. 2008, Ray and Beever 2007), and those that do collect them do not always use them (Conner 1983), or collect comparable amounts of hay (Millar and Zwickel 1972). Most plots in this study contained fewer haypiles than the total number of pikas occupying the plot (e.g., one haypile for four pikas in one location, with few to no observations of haying by individuals lacking haypiles). The haypile likely functions as a backup, in case winter conditions are harsh enough to prevent foraging (Millar and Zwickel 1972, Wilkening et al. 2011). Therefore, if summer conditions were warm enough to prevent sufficient 
haying, and winter conditions prevented or reduced access to forage material, pika survival could be severely diminished.

\section{Specific Activities and Seasonality}

Seasonal differences in this study were inconclusive in all but haying behavior, and another field season with additional months and sites should help to confirm whether there are any seasonal differences in other types of activity between pikas in different populations. It is likely that for pikas in the Sierra, August and September are too similar to cause many behavioral differences. It may be more useful to compare pika behavior in July and October. Haying rates did change between August and September, and this change was different between the sites. The low elevation pikas hayed more in August, and the high elevation pikas hayed more in September. Timing of haying is dependent on precipitation the previous winter, and on the vegetation available (Smith 1974b), so the timing of haying could vary from year to year and between sites.

We observed more foraging in the high elevation than the low elevation site. This may be because temperatures in the high elevation site were low enough to allow pikas to be more surface-active. It is somewhat surprising that more foraging was observed in the high site than the low site because in both elevation classes pikas presumably require a similar amount of forage to maintain daily metabolic rates. If we assume that both populations require a similar amount of forage material, reduced diurnal foraging activity could point to the possibility of unrecorded nocturnal behavior occurring in the low site, or indicate that low elevation pikas may be stressed. 
It is possible that high elevation pikas must eat more to maintain metabolism at colder temperatures and to store more fat before the harsher alpine winter. This assumes, however, that high elevation pikas are experiencing colder winters than low elevation pikas, which may not be the case, in particular because reduced snowpack at lower elevations provide less insulation to pikas under the talus (Beever et al. 2010). Another unlikely explanation is that the nutritional content of available vegetation differs between the two elevation classes. While this may be true, high elevation pikas likely have access to more nutritious vegetation, rather than vice versa, as high sites had an abundance of graminoids, which are less toxic than forbs and shrubs, and are preferred by pikas for immediate consumption (Huntly at al. 1986, Dearing 1997a,b). Lower elevation sites were dominated by shrubs, such as sagebrush (Artemisia tridentata), which tend to have more toxic secondary compounds, and presumably are more difficult for a pika to digest (Shipley et al. 2006). When pikas collect toxic vegetation in haypiles, they do not consume it until the secondary compounds in the vegetation have degraded (after a few months) (Dearing 1997a). How they survive on vegetation with higher concentrations of toxic compounds during the summer is unclear. If it would require that pikas consume more vegetation, then I would expect that low elevation pikas would forage more than high elevation pikas, if they are going to maintain a similar activity level. Since the low elevation pikas foraged less during the day, nocturnal foraging remains a possibility.

\section{Nocturnal Behavior}

Smith (1974b) thought that pikas in his low elevation site were compensating for lower daytime activity levels by collecting haypiles at night. He observed pikas at night 
in both of his sites, and heard a higher frequency of pika calls in the low elevation site. This suggests that the low elevation pikas may be more nocturnal than high elevation pikas. However, Smith (1974b) apparently only went out on one night of full moon at each site, and he rated activity of pikas based on frequency of calls, which is not a true estimate of pika activity. Pika calling is variable among populations and seasons (Barash 1973, Conner 1982, Trefry and Hik 2010). To date, no other studies have been conducted on the nocturnal habits of American pikas at differing elevations.

Pikas have long been considered diurnal, but the question of what they do at night remains unanswered. Afghan pikas (O. rufescens rufescens) studied in the laboratory had highest locomotor activity in early morning and from evening to midnight. This suggests that the Afghan pika is nocturnal (Kosaka et al. 1988). Few comparable studies have been performed on the American pika, but it is possible that American pikas are nocturnal as well. Krear (1965) noted a significant amount of nocturnal alarm calling in his American pika study sites. He believed that pikas called during disturbances, but did not forage at night. To learn more he observed captive pikas, and he noticed that they were active during the night and appeared to eat and drink during that time. More research is needed on the nocturnal behavior of the American pika. For this reason, I ran preliminary tests using remote cameras to see if they could be used to measure nocturnal behavior of pikas in a future study.

My cameras were situated in plots in which the activity of individual pikas was known to be high, in hopes of ensuring successful pictures. However, as I was hoping to capture nocturnal behavior, perhaps I should have put the cameras in plots in which diurnal activity was low. Pikas that do not spend as much time active during the day 
presumably must maintain some level of activity, whether it be foraging or haying, and so they may be more active at night. It is these less diurnally active pikas that could more likely be nocturnal, which may explain why I failed to capture any nighttime photographs of pikas.

It remains uncertain whether cameras can accurately quantify activity rates of pikas. The two cameras that I set up on either side of the same haypile show that very different numbers of pika pictures can be obtained even from the same pika during the same observation session (the two I set up took an average difference of four times the number of pika pictures). This calls into question the accuracy of using cameras to compare activity between sites. I see two possible solutions for this problem. First, in every site that is being photographed there should be two or more cameras set up facing the haypile (or center of activity), in which case an average could be taken of the number of pika pictures produced to calculate an activity rate for the site. Second, the proposed nocturnal study should be extremely large, incorporating a high number of sites with cameras in each, so that the variance in number of pictures taken could be reduced by the very large sample size.

Possibly the biggest problem with using remote cameras to measure activity is camera placement. The easiest place to set up a camera and guarantee pika pictures is in front of a haypile, but this may not be the most accurate location from which to measure nocturnal behavior. Not all pikas produce haypiles, and photographing only those that do may introduce bias. Haypile producing pikas could be more active because they have more "work" to do. Furthermore, their daily activity patterns could be different from those that do not produce haypiles, and they may have less reason to be nocturnal. They 
also may not visit the haypile area at night, even if they are nocturnally active. Predation risk is likely higher for haying pikas than non-haying pikas because pikas travel significantly farther when haying than when foraging (Huntly et al. 1986), and because their flight ability and predator detection may be impaired by the large clump of vegetation they are carrying. Predation risk may increase at night, in part because of a pika's reduced ability to see in the dark (Walls and Judd 1933). Therefore, pikas that hay may limit their haying activities to safer daylight hours, since they are already at higher risk of predation. Additionally, a pika that hays in the day may not have any need to visit the haypile area at night, even if she is active at that time. On the other hand, if a pika does not need to hay, and the risk of foraging in the day is not significantly different from the risk of foraging at night, it may benefit the pika to be active at night. Because she would not be limited by temperature, the pika could possibly go out for longer foraging trips, and be able to eat more, while remaining close to talus and suffering less risk of predation. This could be true for both haying and non-haying pikas.

Unfortunately without a haypile, camera placement becomes more difficult. Pikas do leave other types of sign in high use areas, so cameras could be placed in these areas. Other pika sign includes latrines, fresh scat (Nichols 2010), scattered pieces of vegetation on rocks, and cropped vegetation next to or growing within the talus (Millar and Zwickel 1972). I obtained some pika pictures from a camera facing a high use area, not a haypile, so this is a possibility. With a sufficient number of cameras, perhaps placed throughout a slope in likely high use areas, such as the talus-vegetation interface, and large boulders with small sentry rocks underneath (Millar 2011), the chance of capturing pika activity would increase. Probably the best way to increase the chances of capturing nocturnal 
activity would be to increase the number of cameras and spread them throughout a slope. However, the more cameras, the less feasible the study becomes. More investigations are still needed to determine the best method for measuring pika nocturnal behavior.

Although I did not detect any nocturnal behavior in this study, the occurrence of nocturnal behavior in the American pika is remains a possibility, and is worth investigating in future studies. Probably the greatest suggestion of nocturnal behavior was that I detected less foraging in the low elevation population during the study. This does not point out right to nocturnal behavior, but it does raise some questions, including whether nocturnal foraging is occurring. The benefits of nocturnal foraging could be very high for low elevation pikas. Nocturnal foraging would allow pikas to escape higher diurnal temperatures, as well as releasing them from predation by diurnal predators. Since it has been shown that other species of pikas (O. rufescens rufescens, Kosaka et al. 1988) may be nocturnal, it seems only logical that American pikas, too, should be investigated more thoroughly for nocturnality. With respect to rising temperatures caused by climate change, the need to investigate nocturnal behavior, particularly in low elevation populations, is becoming more pressing. 


\section{Final Remarks}

Although the possibility of behavioral plasticity and even genetic adaptations in pikas in response to changing environmental conditions is promising, environmental changes caused by climate change may occur at a rate beyond which species can adapt (Gienapp et al. 2007, Burger and Lynch 1995). American pikas, which are known to be sensitive to higher temperatures, may have some ability to adapt to rising temperatures, but lower elevation populations are still faced with the possibility of extinction if environmental changes occur before populations can adapt to them. 


\section{CHAPTER 3. TABLES}

Table 1. Pika behaviors recorded.

\begin{tabular}{|l|l|}
\hline Activity & Definition \\
\hline Perch & Sitting \\
\hline Forage & Consuming vegetation \\
\hline Hay & Carrying plant or other material and returning to rocks with the material \\
\hline Run & Moving from place to place (includes walking and hopping) \\
\hline Groom & Cleaning self \\
\hline Visible call & Pika is seen calling (aboveground) \\
\hline Inactive & Pika not in view (presumed under rocks) for at least 30 seconds \\
\hline Out of view & Pika is believed to be active, but is out of sight within the subplot \\
\hline
\end{tabular}

Table 2. Minutes of observation and total numbers of pikas observed in both the high $(3321 \mathrm{~m})$ and low $(2616 \mathrm{~m})$ elevation sites during August and September. Some pikas may overlap between subplots within a single plot, but all subplots contain at least one unique pika.

\begin{tabular}{|l|r|r|}
\hline Month and Site & Minutes of Observation & Number of Pikas \\
\hline August Low & 995.0 & 22 \\
\hline August High & 1041.5 & 27 \\
\hline August Total & 2036.5 & 49 \\
\hline September Low & 919.0 & 16 \\
\hline September High & 1094.0 & 25 \\
\hline September Total & 2013.0 & 41 \\
\hline
\end{tabular}


Table 3. Average temperatures ( $\pm \mathrm{SE}$ ) of high $(3321 \mathrm{~m})$ and low $(2616 \mathrm{~m})$ elevation sites in August and September. Sample sizes (n) are the total number of sampling observations used in the analysis.

\begin{tabular}{|l|r|r|l|}
\hline & \multicolumn{3}{|c|}{ Avg Temp $(\mathrm{C}) \pm$ SE } \\
\hline Site & August $(\mathrm{n}=115)$ & September $(\mathrm{n}=112)$ & Totals \\
\hline High $(\mathrm{n}=120)$ & $17.34 \pm 0.75$ & $13.97 \pm 0.56$ & $15.66 \pm 0.49$ \\
\hline Low $(\mathrm{n}=107)$ & $19.19 \pm 0.93$ & $15.27 \pm 0.90$ & $17.29 \pm 0.67$ \\
\hline Total $(\mathrm{n}=227)$ & $18.22 \pm 0.60$ & $14.58 \pm 0.52$ & $16.42 \pm 0.41$ \\
\hline
\end{tabular}

Table 4. Average activity rates $( \pm \mathrm{SE})$ of pikas in high $(3321 \mathrm{~m})$ and low $(2616 \mathrm{~m})$ elevation sites during August and September sampling periods.

\begin{tabular}{|l|l|l|}
\hline Month & Low Site & High Site \\
\hline August & $0.124 \pm 0.024$ & $0.172 \pm 0.023$ \\
\hline September & $0.187 \pm 0.041$ & $0.175 \pm 0.027$ \\
\hline
\end{tabular}


Table 5. Results of GLMMs predicting pika aboveground activity rate, accounting for plot and subplot as nested random effects. For all models, the degrees freedom (DF) and $P$ values are given for each explanatory variable. $P$ values marked with an asterisk are significant.

\begin{tabular}{|l|c|l|}
\hline 3-way Interaction: & DF & $P$ value \\
\hline Temperature & 1,184 & 0.2825 \\
\hline Site*Month*Time & 3,184 & 0.2313 \\
\hline 2-way Interactions: \\
\hline Temperature & 1,187 & 0.3062 \\
\hline Site*Month & 3,187 & 0.3828 \\
\hline Site*Time & 3,187 & $0.0098^{*}$ \\
\hline Month*Time & 3,187 & 0.3823 \\
\hline Final Model: \\
\hline Temperature & 1,191 & 0.3524 \\
\hline Month & 1,191 & 0.8247 \\
\hline Site*Time & 3,191 & $0.0123^{*}$ \\
\hline
\end{tabular}

Table 6. Results of GLMMs predicting pika forage rate, accounting for plot and subplot as nested random effects. For all models, the degrees freedom (DF) and $P$ values are given for each explanatory variable. $P$ values marked with an asterisk are significant.

\begin{tabular}{|l|l|c|}
\hline 3-way interaction: & DF & \multicolumn{1}{c|}{$\boldsymbol{P}$} \\
\hline Temperature & 1,184 & 0.8675 \\
\hline Site*Month*Time & 3,184 & 0.4127 \\
\hline 2-way interactions: \\
\hline Temperature & 1,187 & 0.9509 \\
\hline Site*Month & 3,187 & 0.9195 \\
\hline Site*Time & 3,187 & 0.2689 \\
\hline Month*Time & 3,187 & 0.2604 \\
\hline \multicolumn{3}{|l|}{ Final Model: } \\
\hline Temperature & 1,194 & 0.3529 \\
\hline Month & 1,194 & 0.4309 \\
\hline Site & 1,8 & $0.0104^{*}$ \\
\hline Time & 3,194 & $0.0324^{*}$ \\
\hline
\end{tabular}


Table 7. Results of GLMMs predicting pika hay rate, accounting for plot and subplot as nested random effects. For all models, the degrees freedom (DF) and $P$ values are given for each explanatory variable. $P$ values marked with an asterisk are significant.

\begin{tabular}{|l|c|c|}
\hline 3-way interaction: & DF & $P$ \\
\hline Temperature & 1,184 & 0.5628 \\
\hline Site*Month*Time & 3,184 & 0.4581 \\
\hline 2-way interactions: \\
\hline Temperature & 1,187 & 0.6037 \\
\hline Site*Month & 3,187 & $0.0445^{*}$ \\
\hline Site*Time & 3,187 & 0.3662 \\
\hline Month*Time & 3,187 & 0.9720 \\
\hline Final Model: & 1,193 & 0.8971 \\
\hline Temperature & 1,193 & $0.0003^{*}$ \\
\hline Time & 1,193 & $0.0539^{*}$ \\
\hline Site*Month
\end{tabular}

Table 8. Results of GLMMs predicting pika run rate, accounting for plot and subplot as nested random effects. For all models, the degrees freedom (DF) and $P$ values are given for each explanatory variable. $P$ values marked with an asterisk are significant.

\begin{tabular}{|l|c|c|}
\hline 3-way interaction: & DF & $P$ \\
\hline Temperature & 1,184 & $0.0639^{*}$ \\
\hline Site*Month*Time & 3,184 & 0.1009 \\
\hline 2-way interactions: \\
\hline Temperature & 1,187 & $0.0675^{*}$ \\
\hline Site*Month & 3,187 & 0.7355 \\
\hline Site*Time & 3,187 & $0.0090^{*}$ \\
\hline Month*Time & 3,187 & 0.1940 \\
\hline Final Model: & 1,191 & 0.1513 \\
\hline Temperature & 1,191 & 0.5183 \\
\hline Month & 3,191 & $0.0167^{*}$ \\
\hline Site*Time
\end{tabular}


Table 9. Results of GLMMs predicting pika perch rate, accounting for plot and subplot as nested random effects. For all models, the degrees freedom (DF) and P values are given for each explanatory variable. $\mathrm{P}$ values marked with an asterisk are significant.

\begin{tabular}{|l|c|c|}
\hline 3-way interaction: & DF & $\boldsymbol{P}$ \\
\hline Temperature & 1,184 & 0.5103 \\
\hline Site*Month*Time & 3,184 & 0.2860 \\
\hline 2-way interactions: \\
\hline Temperature & 1,187 & 0.5542 \\
\hline Site*Month & 3,187 & 0.3450 \\
\hline Site*Time & 3,187 & $0.0630^{*}$ \\
\hline Month*Time & 3,187 & 0.5935 \\
\hline Final Model: & 1,191 & 0.5036 \\
\hline Temperature & 1,191 & 0.7828 \\
\hline Month & 3,191 & $0.0649^{*}$ \\
\hline Site*Time
\end{tabular}

Table 10. Results of GLMMs predicting pika groom rate, accounting for plot and subplot as nested random effects. For all models, the degrees freedom (DF) and $P$ values are given for each explanatory variable.

\begin{tabular}{|l|l|l|}
\hline 3-way interaction: & DF & $\boldsymbol{P}$ \\
\hline Temperature & 1,184 & 0.3492 \\
\hline Site*Month*Time & 3,184 & 0.2798 \\
\hline 2-way interactions: & \multicolumn{2}{|l|}{} \\
\hline Temperature & 1,187 & 0.2666 \\
\hline Site*Month & 3,187 & 0.4082 \\
\hline Site*Time & 3,187 & 0.2381 \\
\hline Month*Time & 3,187 & 0.4545 \\
\hline Final Model: & 1,194 & 0.5820 \\
\hline Temperature & 1,194 & 0.9458 \\
\hline Month & 1,8 & 0.6572 \\
\hline Site & 3,194 & 0.6357 \\
\hline Time
\end{tabular}




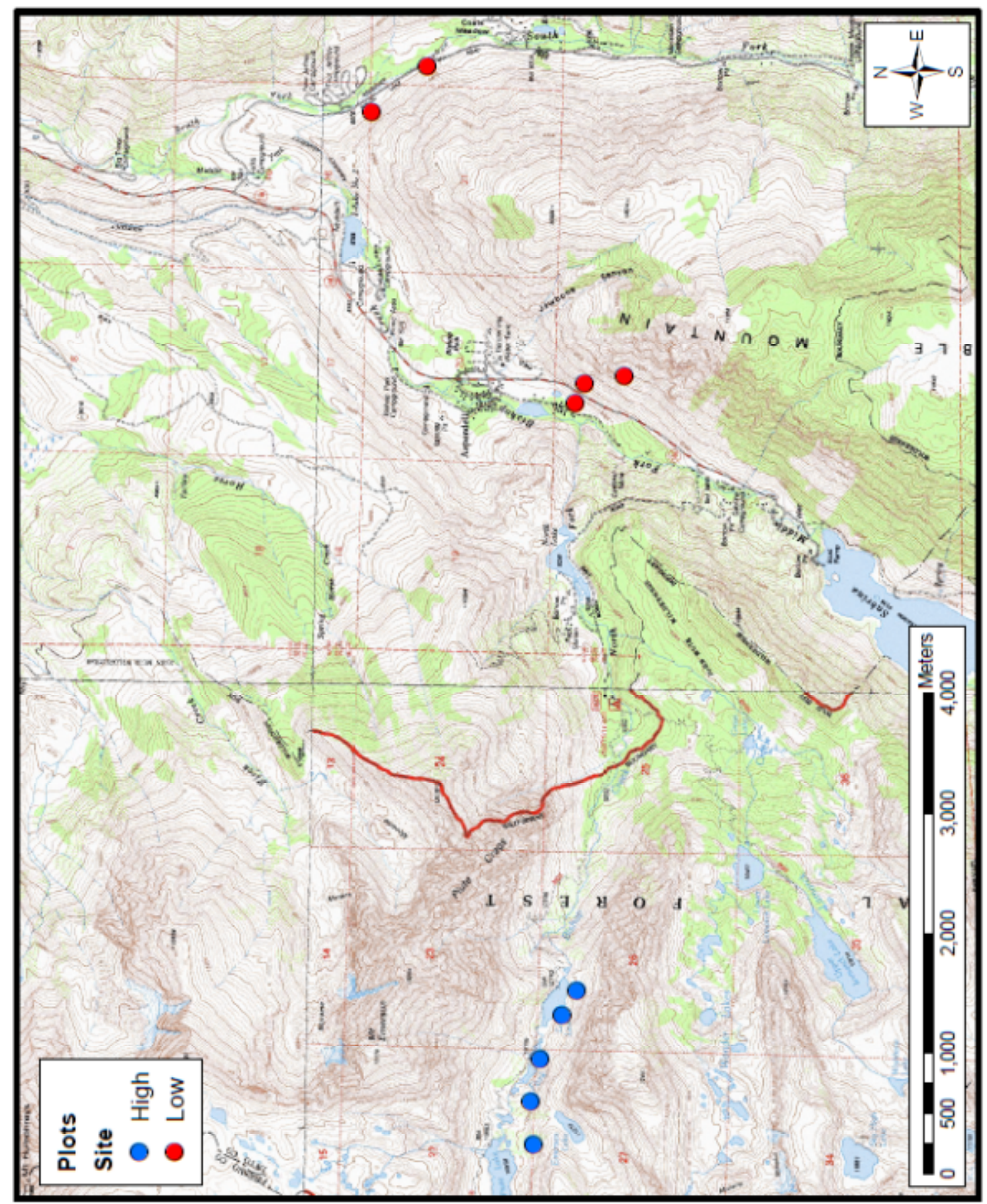

Figure 1. Map of study area, showing plots in both high $(3321 \mathrm{~m})$ and low $(2616 \mathrm{~m})$ elevation sites. Plots are not drawn to scale. 


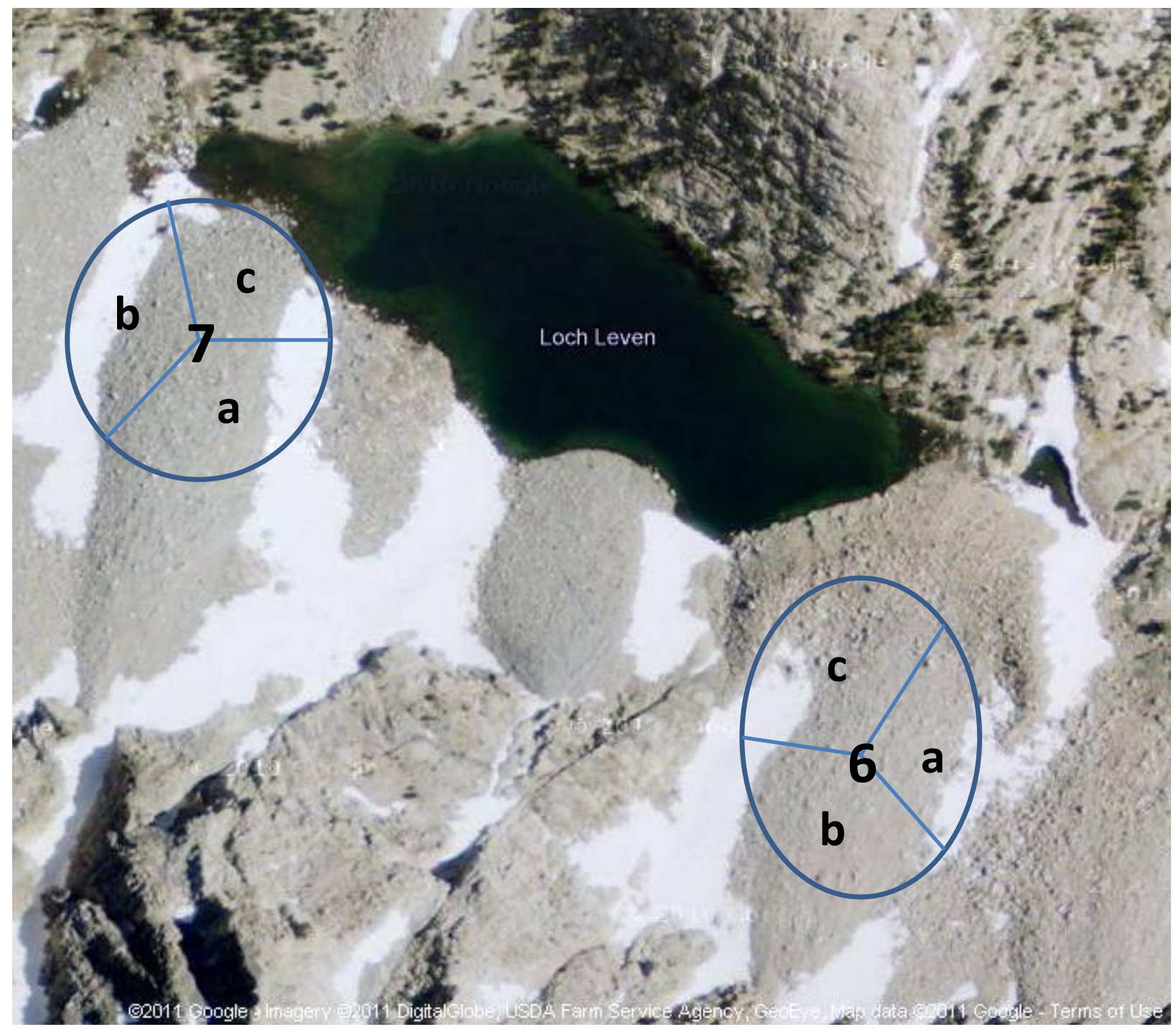

Figure 2. Map of plots 6 and 7, in the high elevation site, near Loch Leven in the Inyo National Forest. These plots are approximately $250 \mathrm{~m}$ apart. The numbers " 6 " and " 7 " mark the center of each plot, at approximately the location that observers used to view pikas. Each of the subplots, a-c, contained at least one unique pika. 

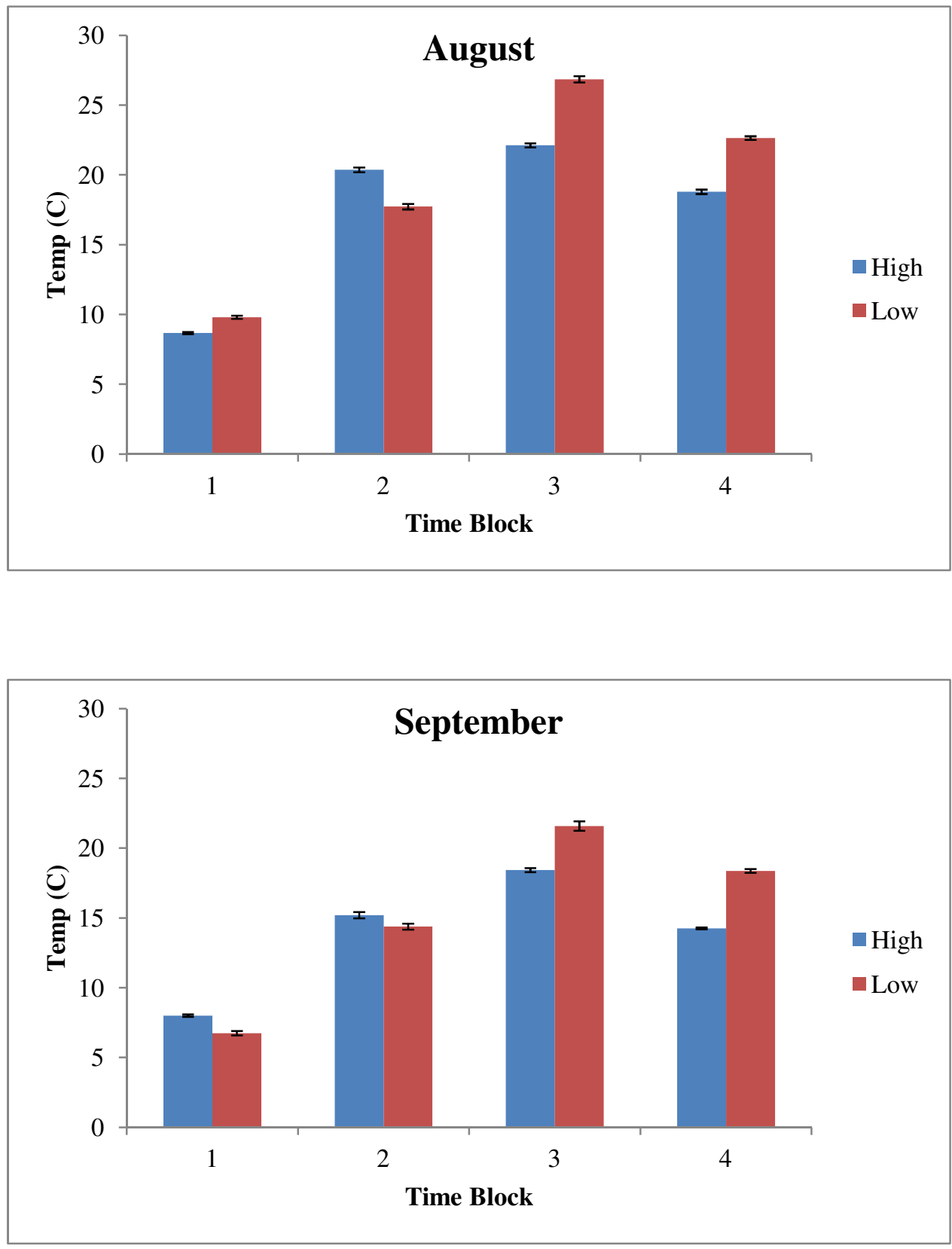

Figure 3. Average temperatures $( \pm \mathrm{SE})$ on shaded talus surfaces during pika observation sessions in August and September, in the high $(3321 \mathrm{~m})$ and low $(2616 \mathrm{~m})$ elevation site throughout the day, from time blocks 1-4 (dawn to dusk). 


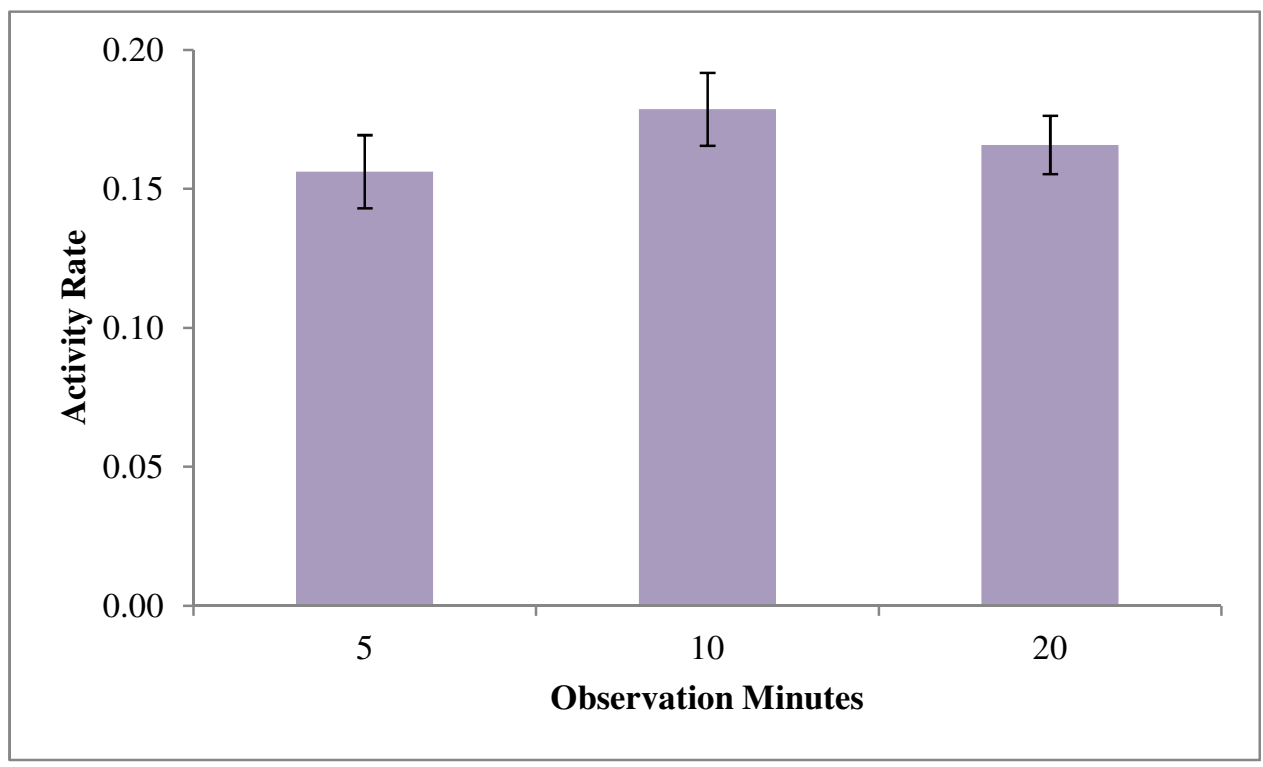

Figure 4. Average pika activity rates $( \pm \mathrm{SE})$ during the first five, second five (10, graph), and final ten minutes (20, graph) of observation sessions.

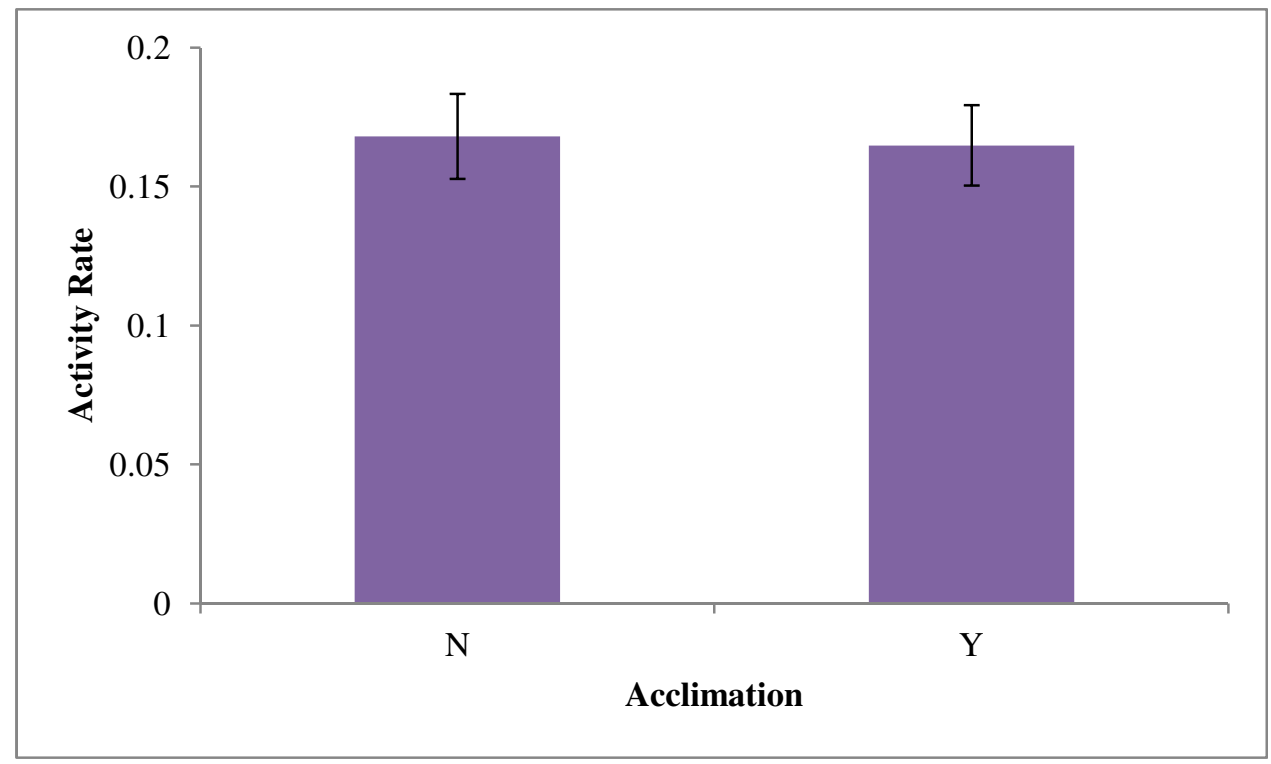

Figure 5. Average pika activity rates $( \pm S E)$ in samples without an acclimation period, $N$ (observers waited at least ten minutes before observations began), and samples with an acclimation period, Y (observers began sampling immediately upon arrival to slope). 

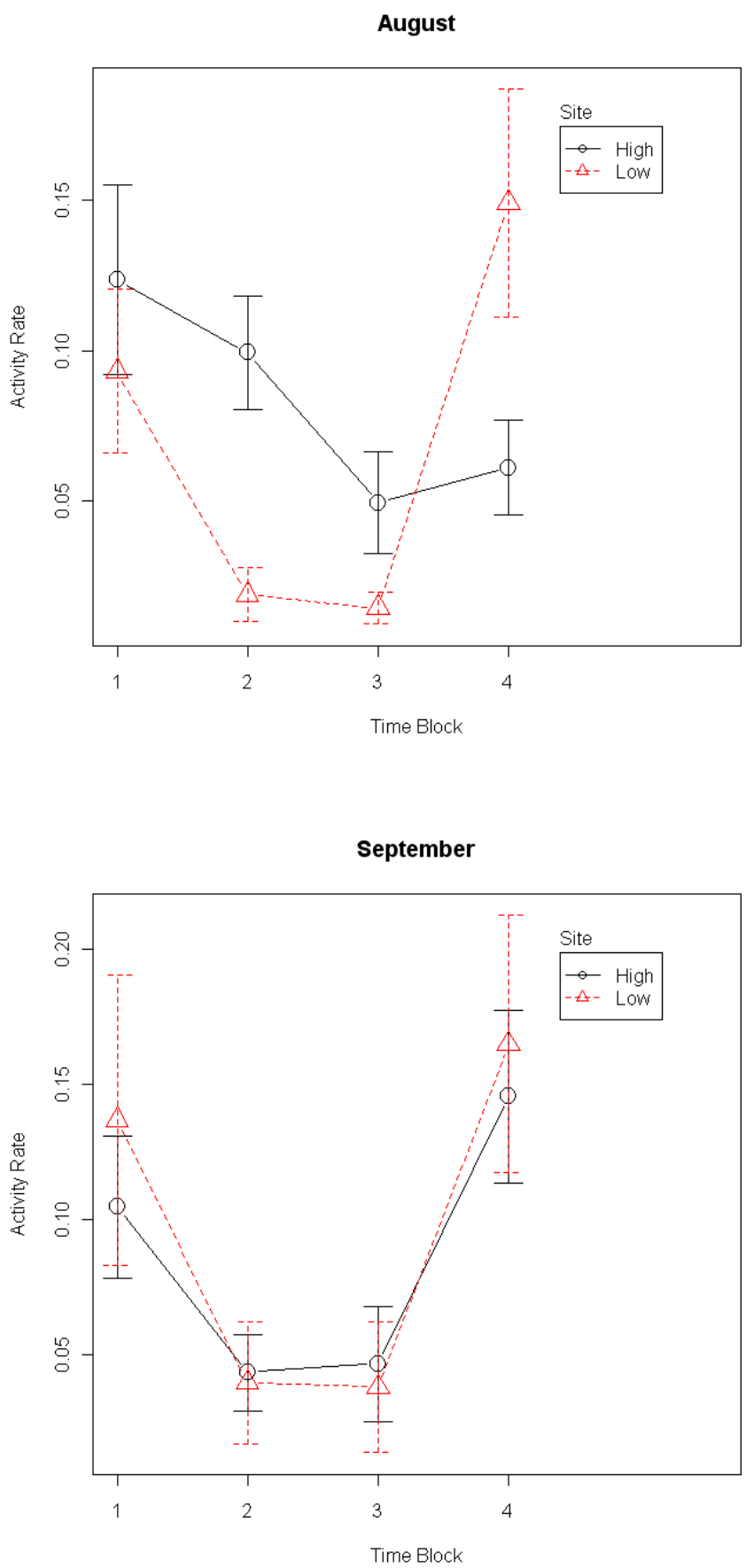

Figure 6. Average pika aboveground activity rate $( \pm \mathrm{SE})$ throughout the day in August and September in a high $(3321 \mathrm{~m})$ and a low $(2616 \mathrm{~m})$ elevation site. Time periods are time blocks 1 through 4 , beginning at dawn and ending at dusk. 


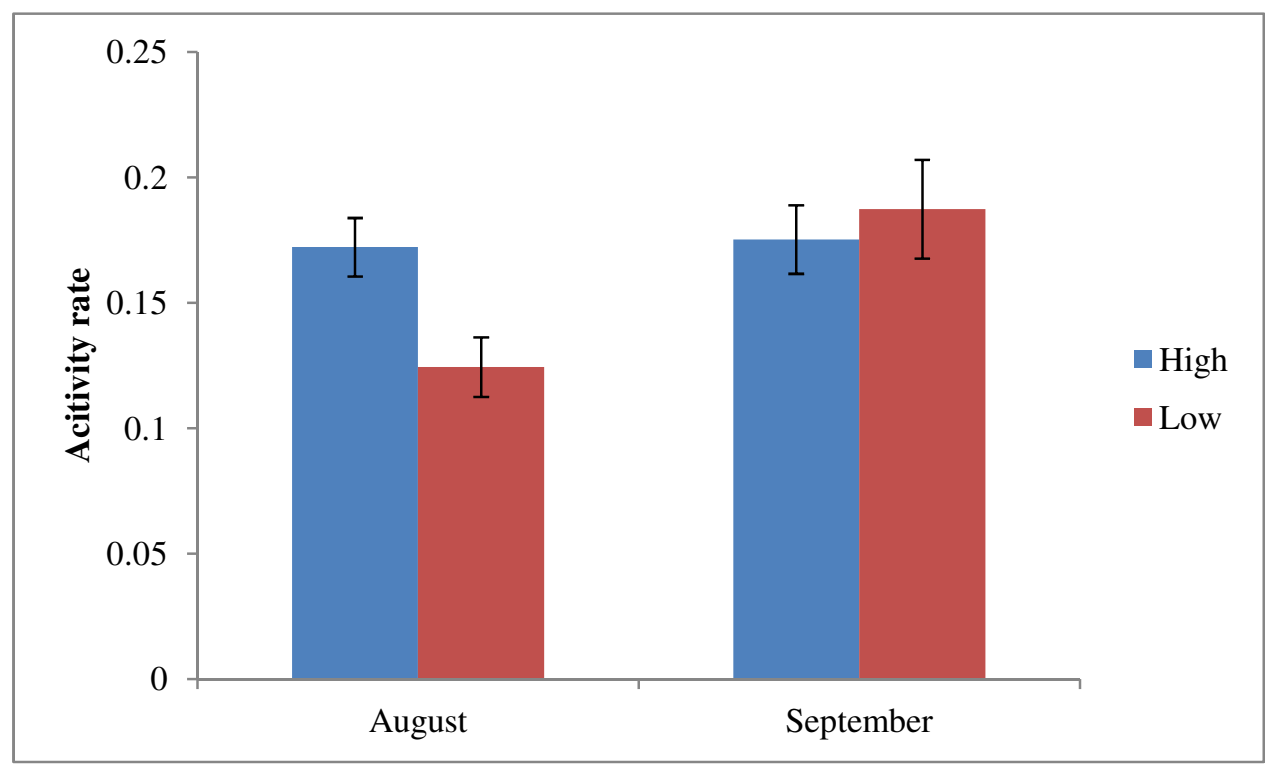

Figure 7. Average aboveground activity rates $( \pm \mathrm{SE})$, per $30 \mathrm{sec}$, of pikas in the high (3321 m) and low (2616 m) elevation sites during August and September. 

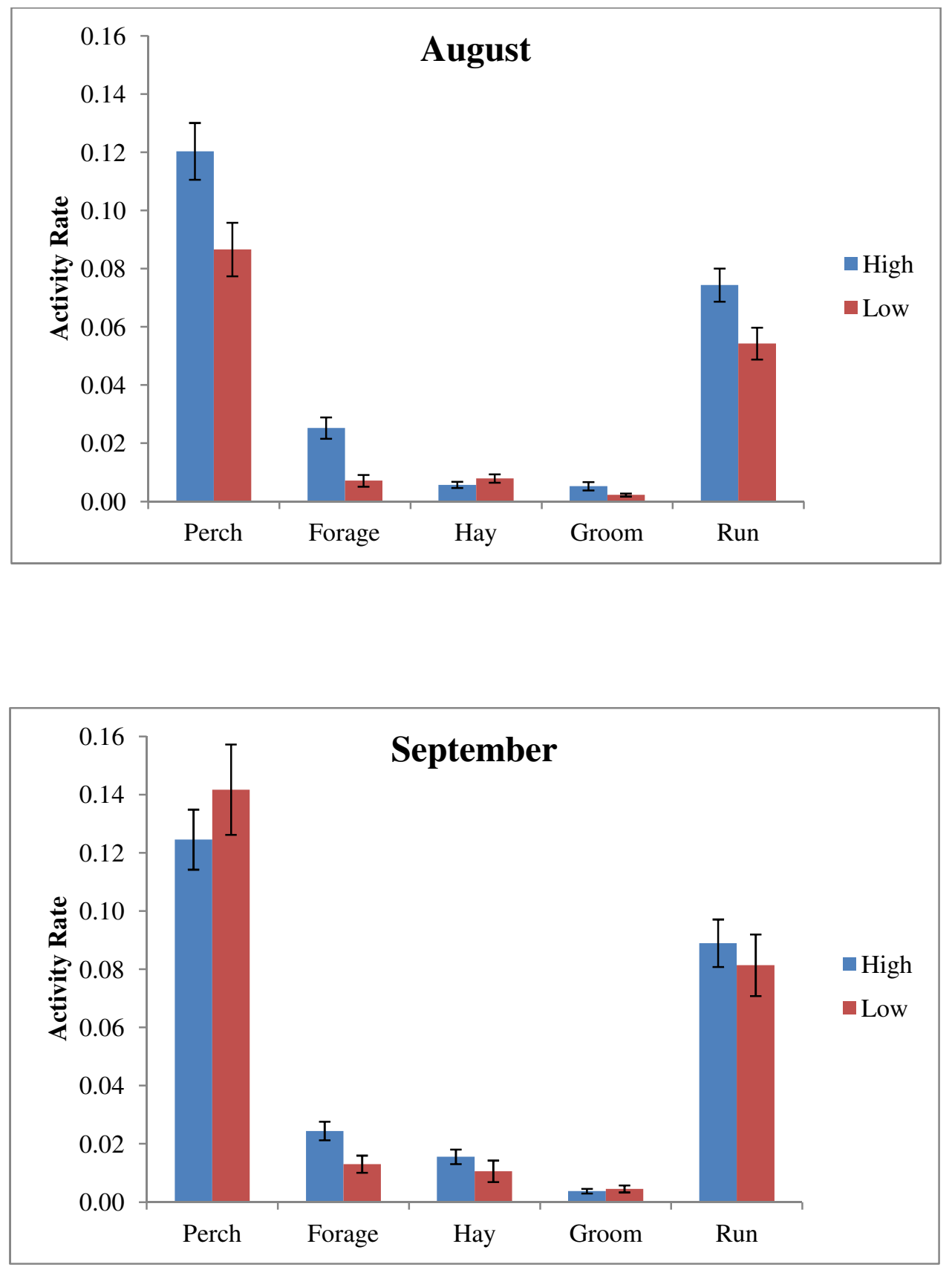

Figure 8. Rates of specific activities $( \pm \mathrm{SE})$ in the high $(3321 \mathrm{~m})$ and low $(2616 \mathrm{~m})$ elevation sites, in August and September. 


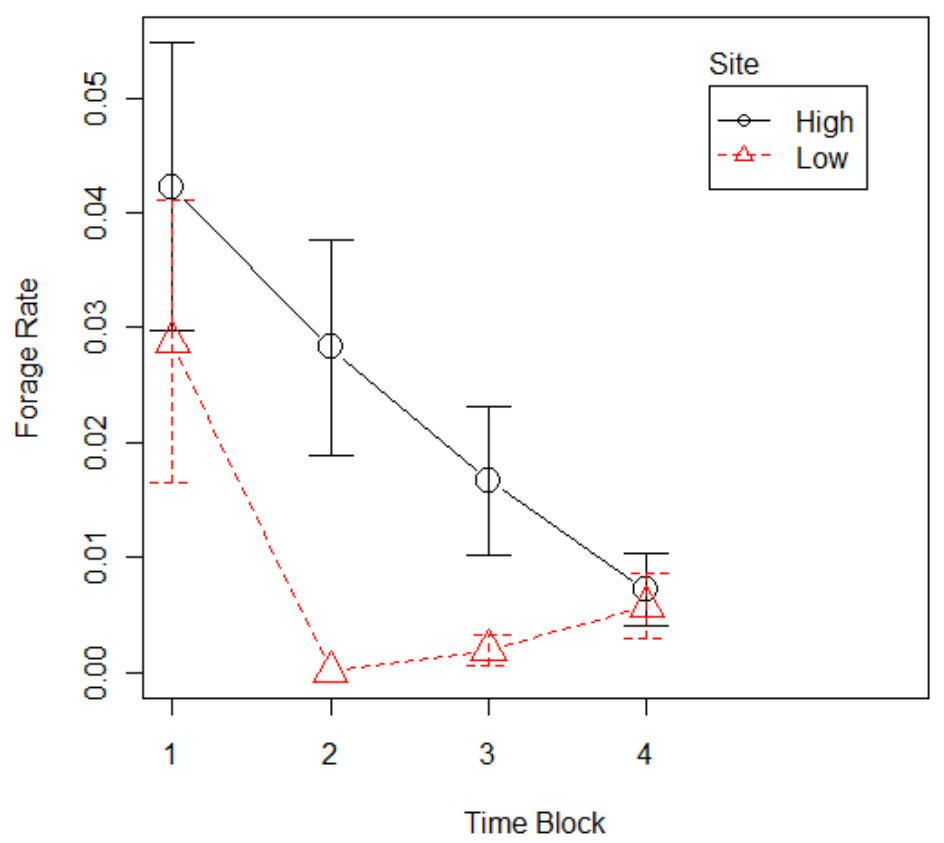

Figure 9. Observed rates of foraging $( \pm S E)$ in high $(3321 \mathrm{~m})$ and low $(2616 \mathrm{~m})$ elevation sites in time blocks 1-4 (dawn to dusk). 


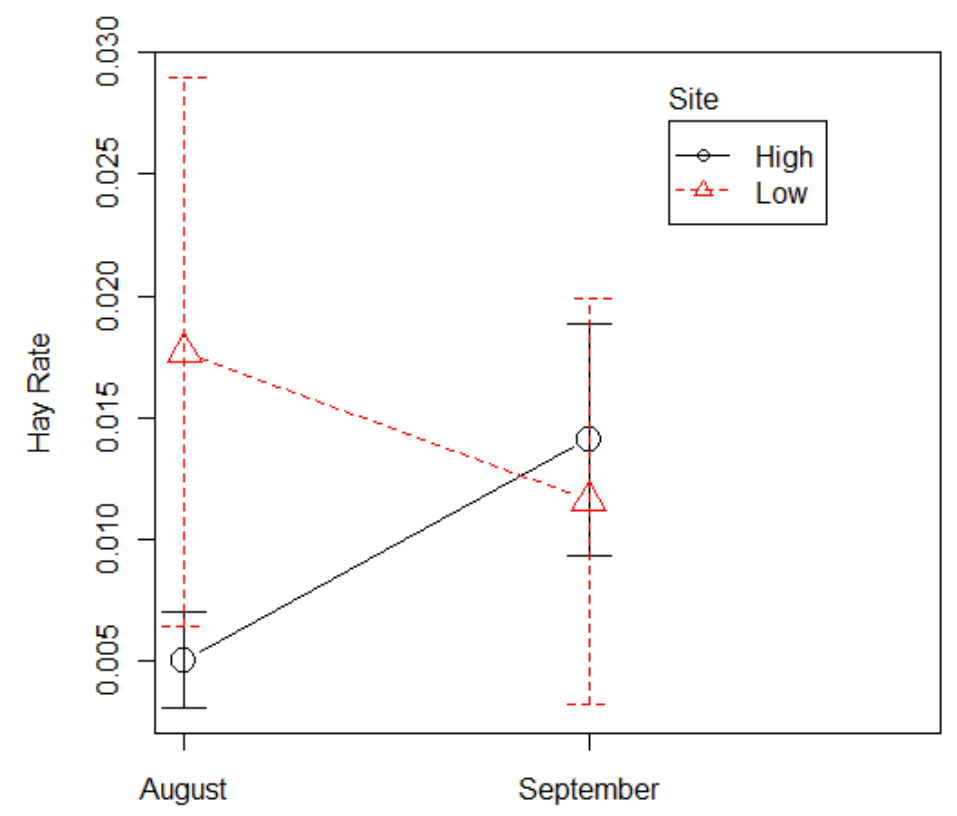

Figure 10. Observed rates of haying $( \pm \mathrm{SE})$ in high $(3321 \mathrm{~m})$ and low $(2616 \mathrm{~m})$ elevation sites during August and September. 


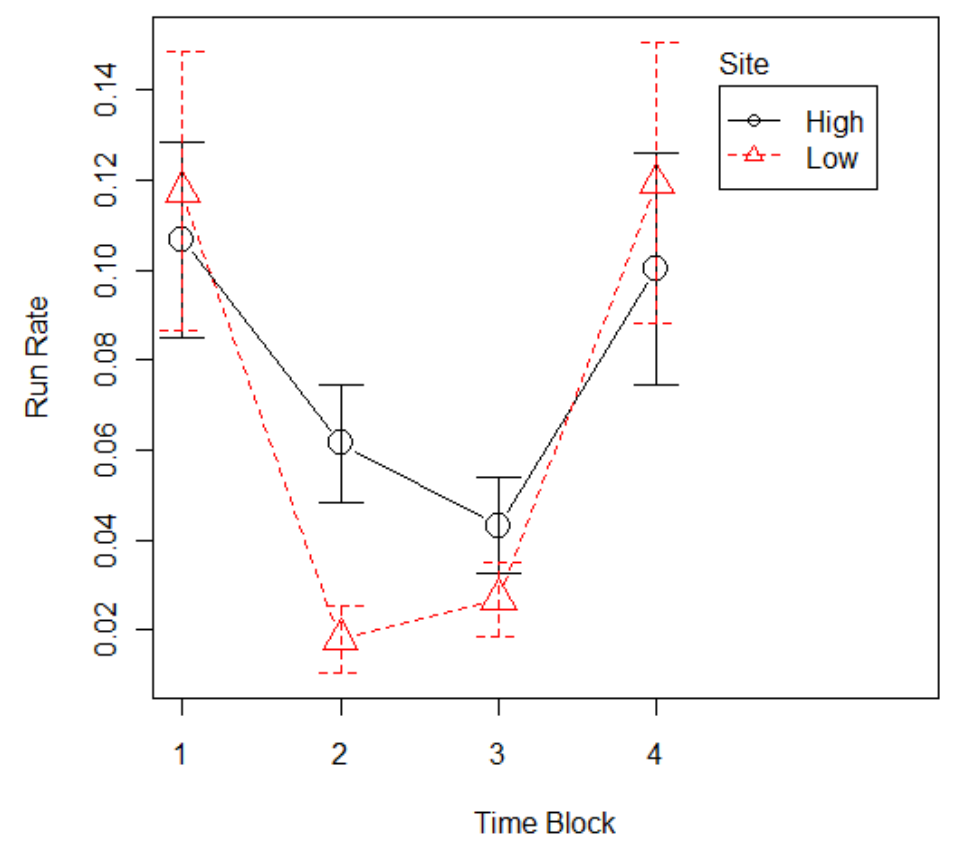

Figure 11. Observed rates of running $( \pm \mathrm{SE})$ in high $(3321 \mathrm{~m})$ and low $(2616 \mathrm{~m})$ elevation sites in time blocks 1-4 (dawn to dusk). 


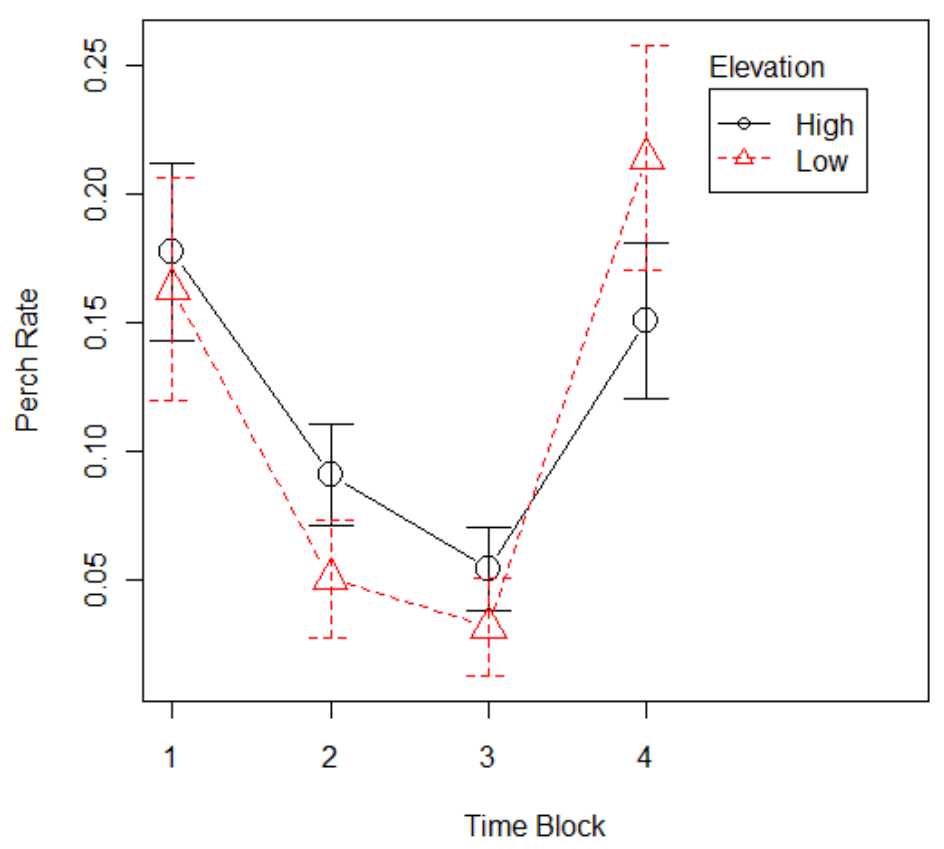

Figure 12. Observed rates of perching $( \pm \mathrm{SE})$ in high $(3321 \mathrm{~m})$ and low $(2616 \mathrm{~m})$ elevation sites in time blocks 1-4 (dawn to dusk). 


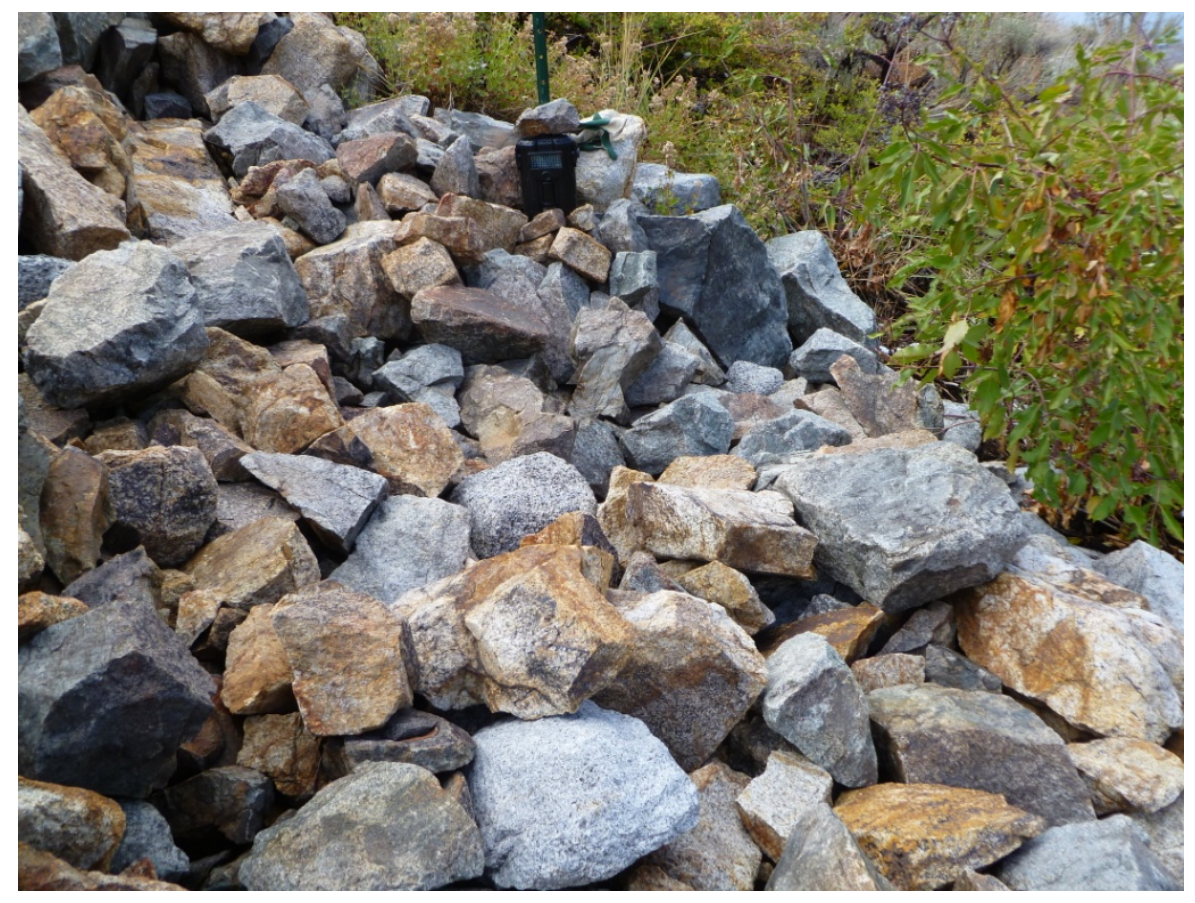

A.

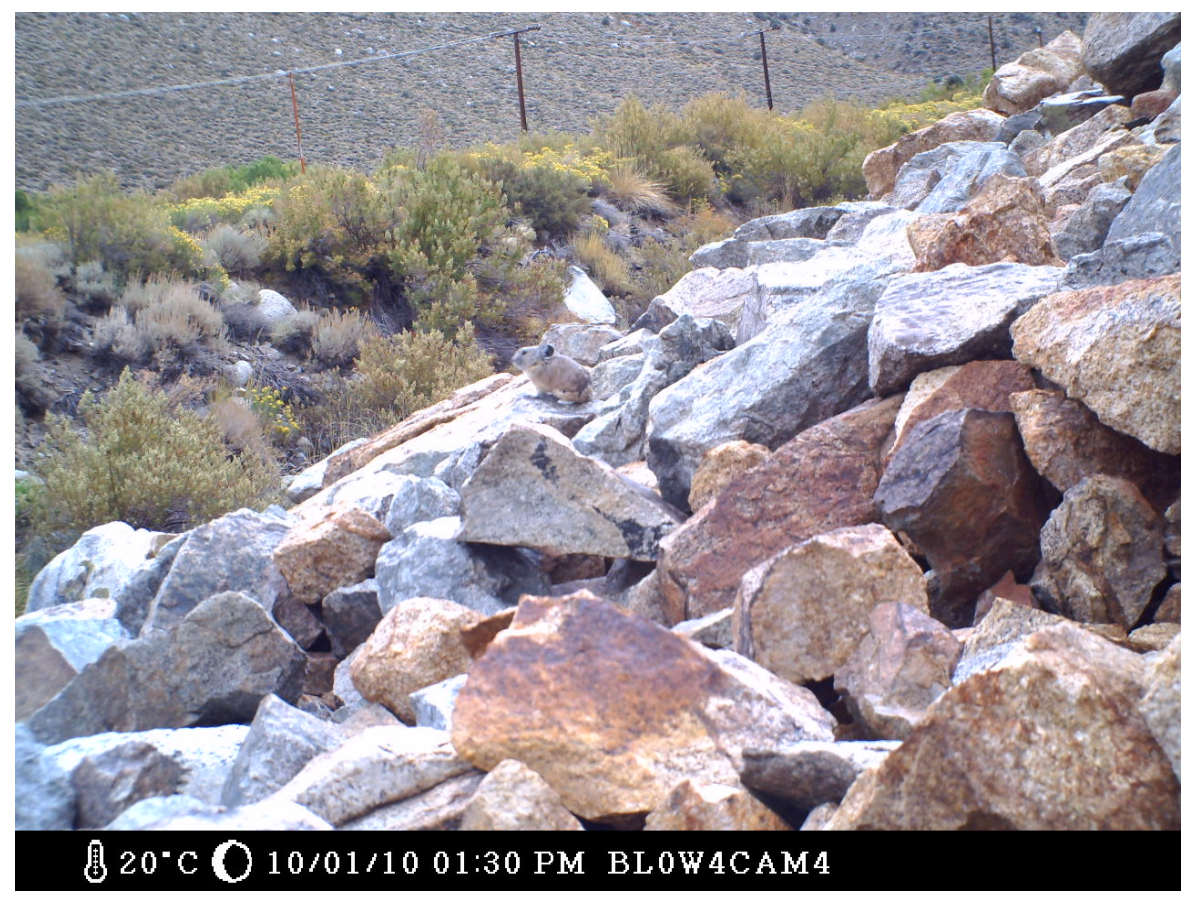

B.

Figure 13. (A) Camera placed approximately $4 \mathrm{~m}$ from area of high activity, resulted in successful pika pictures. (B) Successful pika picture resulting from this camera. 


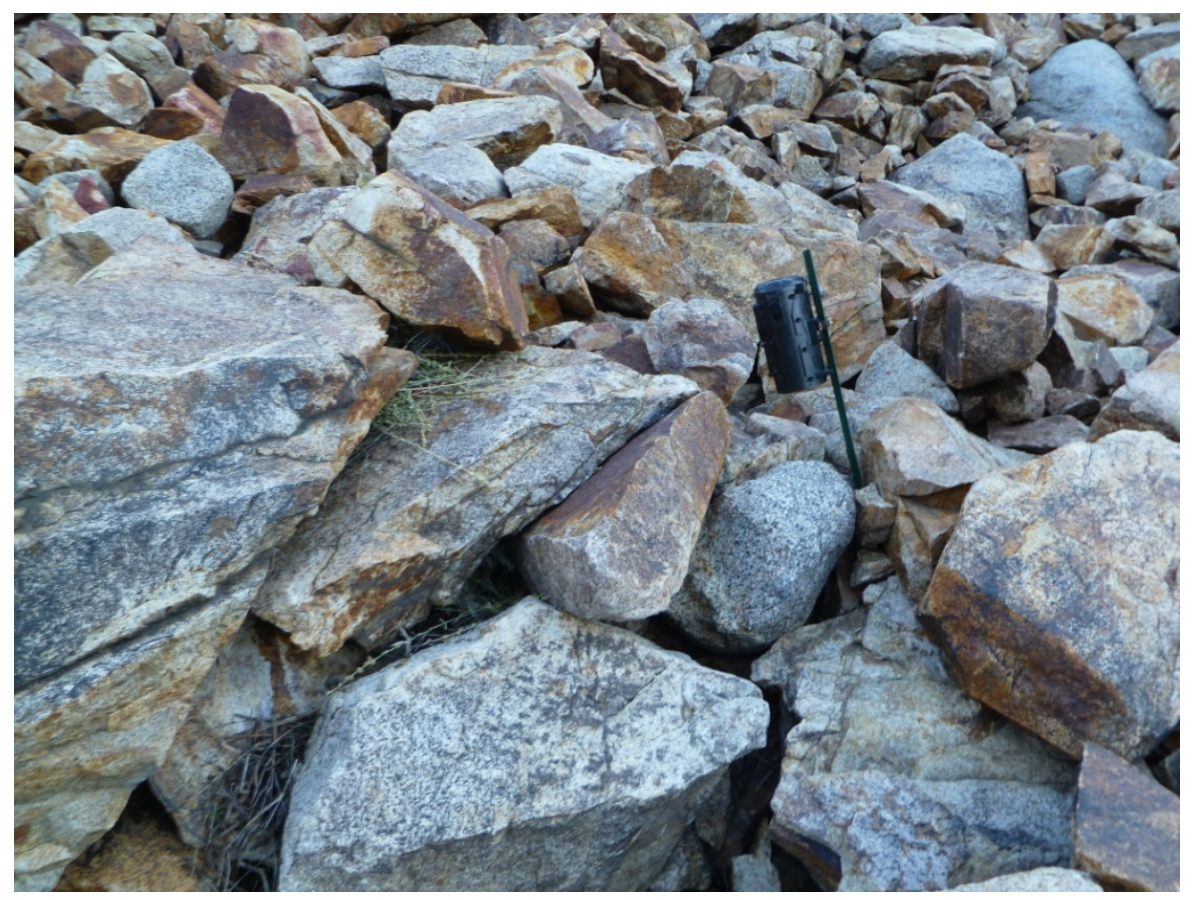

Figure 14. Camera placed approximately $1 \mathrm{~m}$ from haypile. All pictures produced were extremely blurry. Vegetation protruding from rocks is the haypile. 


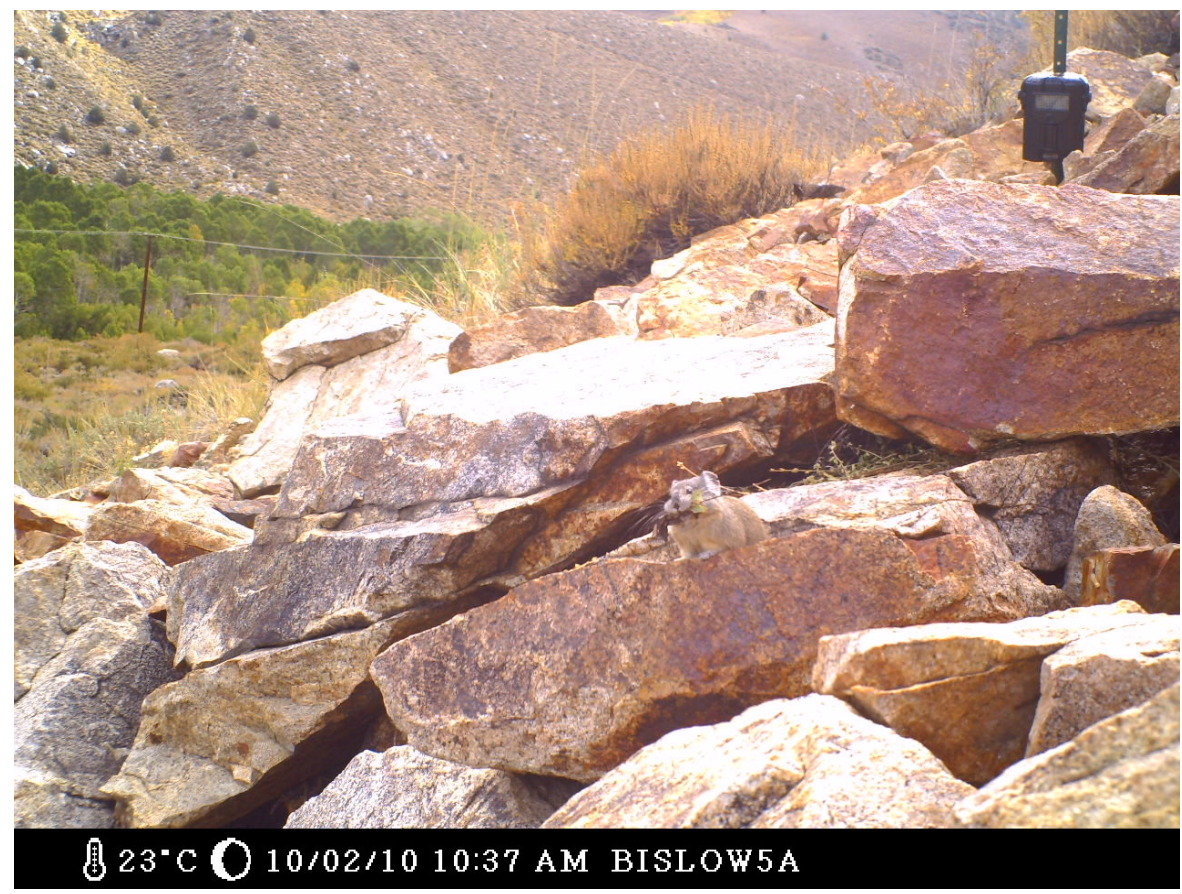

Figure 15. Successful pika picture. Note the camera opposite haypile. Both cameras were successful in photographing pikas, but one (pictured) took four times fewer pictures than the other. Vegetation protruding from rocks is the haypile. 


\section{CHAPTER 3. LIST OF REFERENCES}

Barash, D.P. 1973. Territorial and foraging behavior of pika (Ochotona princeps) in Montana. American Midland Naturalist 89:202-207.

Beever, E.A. 2002. Persistence of pikas in two low-elevation national monuments in the western United States. Park Science 21:23-29.

Beever, E.A., P.F. Brussard, and J. Berger. 2003. Patterns of extirpation among isolated populations of pikas (Ochotona princeps) in the Great Basin. Journal of Mammalogy 84:37-54.

Beever, E.A., J.L. Wilkening, D.E. McIvor, S.S. Weber, and P.F. Brussard. 2008. American pikas (Ochotona princeps) in northwestern Nevada: a newly discovered population at a low-elevation site. Western North American Naturalist 68:8-14.

Beever, E.A., C. Ray, P.W. Mote, J.L. Wilkening. 2010. Testing alternative methods of climate-mediated extirpations. Ecological Society of America 20:164-178.

Beniston, M. 2003. Climatic change in mountain regions: a review of possible impacts. Climatic Change 59:5-31.

Burger, R. and M. Lynch. 1995. Evolution and extinction in a changing environment: a quantitative-genetic analysis. Evolution 49:151-163.

Conner, D. 1982. Geographic variation in short calls of pika (Ochotona princeps). Journal of Mammalogy 63:48-52.

Conner, D. 1983. Seasonal changes in activity patterns and the adaptive value of haying in pikas (Ochotona princeps). Canadian Journal of Zoology 61:411-416.

Crawley, M.J. 2007. The R book. John Wiley \& Sons Ltd., West Sussex, England.

Dearing, M.D. 1997a. The manipulation of plant toxins by a food-hoarding herbivore, Ochotona princeps. Ecology 78:774-781.

Dearing, M.D. 1997b. The function of haypiles of pikas (Ochotona princeps). Journal of Mammalogy 78:1156-1163.

Franken, R.J. and D.S. Hik. 2004. Influence of habitat quality, patch size and connectivity on colonization and extinction dynamics of collared pikas Ochotona collaris. Journal of Animal Ecology 73:889-896. 
Gienapp, P., C. Teplistky, J.S. Alho, J.A. Mills, and J. Merilä. 2008. Climate change and evolution: disentangling environmental and genetic responses. Molecular Ecology 17:167-178.

Gross, K., G. Pasinelli, and H.P. Kunc. 2010. Behavioral plasticity allows short-term adjustment to a novel environment. American Naturalist 176:456-464.

Hayes, A.R. and N.J. Huntly. 2005. Effects of wind on the behavior and call transmission of pikas (Ochotona princeps). Journal of Mammalogy 86:974-981.

Holmes, W.G. 1991. Predator risk affects foraging behavior of pikas: observation and experimental evidence. Animal Behaviour: 42:111-119.

Huntly, N.J., A.T. Smith, and B.L. Ivins. 1986. Foraging behavior of the pika (Ochotona princeps) with comparisons of grazing versus haying. Journal of Mammalogy 67:139148.

Intergovernmental Panel on Climate Change (IPCC), 2007. Climate change impacts, adaptation, and vulnerability. In: Contribution of Working Group II to the Fourth Assessment Report of the IPCC. Cambridge University Press, Cambridge, UK.

Ivins, B.L. and A.T. Smith. 1983. Responses of pikas (Ochotona princeps, Lagomorpha) to naturally occurring terrestrial predators. Behavioral Ecology and Sociobiology 13:277285.

Jeffress, M., M. Britten, and S. Wolff. 2010. Pikas in peril, mulitregional vulnerability assessment of a climate-sentinel species. National Park Service U.S. Department of the Interior. http://science.nature.nps.gov/im/units/ucbn/monitor/pika/pika_peril/docs/PikasInPeril_Re sourceBrief_20100928.pdf

Kawamichi, T. 1976. Hay territory and dominance rank of pikas (Ochotona princeps). Journal of Mammalogy 57:133-148.

Kosaka, M., G.J. Yang, T. Matsumoto, N. Ohwatari, K. Tsuchiya, C.M. Chen, K. Nakamura, S. Matsuo, and T. Moriuchi. 1988. Analysis of locomotor activity on pika (whistle rabbit). Tropical Medicine 30:213-218.

Krear, H.R. 1965. An ecological and ethological study of the pika (Ochotona princeps saxatilis bangs) in the front range of Colorado. Ph.D. Thesis. University of Colorado, Boulder.

Lutton, L.M. 1975. Notes on the territorial behavior and response to predators of the pika, Ochotona princeps. Journal of Mammalogy 56:231-234.

MacArthur, R.A. and L.C.H. Wang. 1973. Physiology of thermoregulation in the pika, Ochotona princeps. Canadian Journal of Zoology 51:11-16. 
MacArthur, R.A. and L.C.H. Wang. 1974. Behavioral thermoregulation in the pika, Ochotona princeps: a field study using radiotelemetry. Canadian Journal of Zoology 52:353-358.

McKechnie, A.M., A.T. Smith, and M.M Peacock. 1994. Kleptoparasitism in pikas (Ochotona princeps): theft of hay. Journal of Mammalogy 75:488-491.

Millar, C. USDA Forest Service. Pika Survey Instruction form (online). Accessed Jan. 3, 2011 at http://www.fs.fed.us/psw/publications/millar/PikaSurvey_Instructions_Form.pdf

Millar, C.I. and R.D. Westfall. 2010. Distribution and climatic relationships of the American pika (Ochotona princeps) in the Sierra Nevada and western Great Basin, USA; periglacial landforms as refugia in warming climates. Arctic, Antarctic, and Alpine Research 42:76-88.

Millar, J.S. and F.C. Zwickel. 1972. Characteristics and ecological significance of hay piles of pikas. Mammalia 36: 657-667.

Moilanen, A., A.T. Smith and I. Hanski. 1998. Long-term dynamics in a metapopulation of the American pika. The American Naturalist 152:530-542.

Naftz, D.L., D.D. Susong, P.F. Schuster, L.D. Cecil, M.D. Dettinger, R.L. Michel, and C. Kendall. 2002. Ice core evidence of rapid air temperature increases since 1960 in alpine areas of the Wind River Range, Wyoming, United States. Journal of Geophysical Research 107:10-29.

Nichols, L.B. 2010. Fecal pellets in American pikas (Ochotona princeps) provide a crude chronometer for dating patch occupancy. Western North American Naturalist 70:500507.

Parmesan, C. and G. Yohe. 2003. A globally coherent fingerprint of climate impacts across natural systems. Nature 421:37-42.

Peacock, M.M. 1997. Determining natal dispersal patterns in a population of North American pikas (Ochotona princeps) using direct mark-resight and indirect genetic methods. Behavioral Ecology 3:340-350.

Price, T.D, A. Qvarnström, and D.E. Irwin. 2003. The role of phenotypic plasticity in driving genetic evolution. Proceedings: Biological Sciences 270:1433-1440.

Ray, C., Beever, E., 2007. Distribution and abundance of the American Pika (Ochotona princeps) within Lava Beds National Monument. National Park Service Report, 1-57.

Roach, W.J., N. Huntly, and R. Inouye. 2001. Talus fragmentation mitigates the effects of pikas, Ochotona princeps, on high alpine meadows. Oikos 92:315-324. 
Rodhouse, T.J., E.A. Beever, L.K Garrett, K.M. Irvine, M.R. Jeffress, M. Munts, and C. Ray. 2010. Distribution of American pikas in a low-elevation lava landscape: conservation implications from the range periphery. Journal of Mammalogy 91:12871299.

Shipley, L.A., T.B. Davila, N.J. Thines, and B.A. Elias. 2006. Nutritional requirements and diet choices of the pygmy rabbit (Brachylagus idahoensis): a sagebrush specialist. Journal of Chemical Ecology 32:2455-2474.

Smith, A.T. 1974a. The distribution and dispersal of pikas: consequences of insular population structure. Ecology 55:1112-1119.

Smith, A.T. 1974b. The distribution and dispersal of pikas: influences of behavior and climate. Ecology 55:1368-1376.

Smith, A.T. and B.L. Ivins. 1983. Colonization in a pika population: dispersal vs philopatry. Behav Ecol Sociobiol 13:37-47.

Smith, A.T. and M.L. Weston. 1990. Mammalian Species, Ochotona princeps. Journal of Mammalogy 352:1-8.

Svendsen, G.E. 1979. Territoriality and behavior in a population of pikas (Ochotona princeps). Journal of Mammalogy 60:324-330.

Trefry, S.A. and D.S. Hik. 2010. Variations in pika (Ochotona collaris, O. princeps) vocalizations within and between populations. Ecography 33:784-795.

Tyser, R.W. 1980. Use of substrate for surveillance behaviors in a community of talus slope mammals. American Midland Naturalist 104:32-38.

Walls, G.L. and H.D. Judd. 1933. The intra-ocular colour-filters of vertebrates. The British Journal of Opthalmology November:641-675.

Walther, G.-R., E. Post, P. Convey, A. Menzel, C. Parmesan, T.J.C. Beebee, J.-M. Fromentin, O. Hoegh-Guldberg, and F. Bairlein. 2002. Ecological responses to recent climate change. Nature 416:389-395.

Warton, D.I. and F.K. Hui. 2011. Arcsine is asinine: the analysis of proportions in ecology. Ecology 92:3-10.

Wilkening, J.L., C. Ray, E.A. Beever, and P.F. Brussard. 2011. Modeling contemporary range retraction in Great Basin pikas (Ochotona princeps) using data on microclimate and microhabitat. Quaternary International 235:77-88. 
Wolf, S., B. Nowicki, and K. Siegel. 2007a. Before the California Fish and Game Commission: Petition to list the American pika (Ochotona princeps) as threatened under the California Endangered Species Act. Center for Biological Diversity.

http://www.biologicaldiversity.org/species/mammals/American_pika/pdfs/petition_ca2007.pdf

Wolf, S., B. Nowicki, and K. Siegel. 2007b. Before the Secretary of the Interior: Petition to list the American pika (Ochotona princeps) as threatened or endangered under the United States Endangered Species Act. Center for Biological Diversity. http://www.biologicaldiversity.org/species/mammals/American_pika/pdfs/Americanpika-federal-petition-10-01-2007.pdf 